Supporting Information for

\title{
Charge Transfer and Spin Dynamics in a Zinc Porphyrin Donor Covalently Linked to One or Two Naphthalenediimide Acceptors
}

Laura Bancroft, ${ }^{1}$ Jinyuan Zhang, ${ }^{1}$ Samantha M. Harvey, ${ }^{1}$ Matthew D. Krzyaniak, ${ }^{1}$ Peng Zhang, ${ }^{2}$ Richard D. Schaller, ${ }^{1,4}$ David N. Beratan, ${ }^{2,3^{*}}$ Ryan M. Young, ${ }^{1, *}$ and Michael R. Wasielewski ${ }^{1, *}$

${ }^{1}$ Department of Chemistry and Institute for Sustainability and Energy at Northwestern, Northwestern University, Evanston, IL 60208-3113, USA

${ }^{2}$ Department of Chemistry, Duke University, Durham, NC 27708

${ }^{3}$ Departments of Biochemistry and Physics, Duke University, Durham, NC 27708

${ }^{4}$ Center for Nanoscale Materials, Argonne National Laboratory, Lemont, IL 60439, USA

*Correspondence to:m-wasielewski@northwestern.edu; ryan.young@northwestern.edu; david.beratan@duke.edu

\section{Contents}

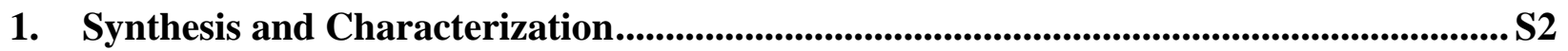

2. Visible and NIR Transient Absorption Spectroscopy ..............................................S6

3. Transient Femtosecond IR Spectroscopy .................................................................S12

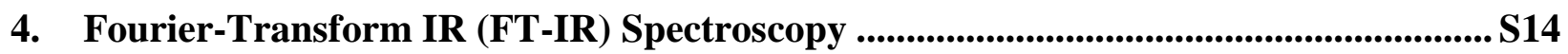

5. Calculation of Free Energies of Reaction and Reorganization Energies ......................S15

6. Estimation of Observed ${ }^{3 *} \mathrm{ZnP}$ Yield in $295 \mathrm{~K}$ Toluene ..............................................S16

7. Magnetic Field Effect Transient Absorption Experiments..............................................S16

8. Results of DFT and TDDFT Computations .................................................................S17

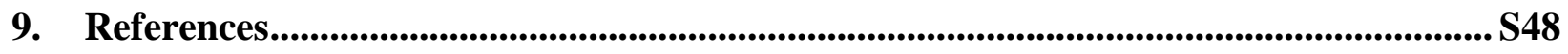




\section{Synthesis and Characterization}

Reagents and solvents were purchased from commercial sources and used as received unless otherwise noted. Compounds $\mathbf{A}, \mathbf{B}$, and $\mathbf{C}$ were synthesized according to existing literature procedures. ${ }^{1-2}{ }^{1} \mathrm{H}$ and ${ }^{13} \mathrm{C}$ NMR spectra were recorded on a Varian $500 \mathrm{MHz}$ spectrometer at room temperature. ${ }^{1} \mathrm{H}$ and ${ }^{13} \mathrm{C}$ chemical shifts are listed in parts per million (ppm) and are referenced to residual protons or carbons of the deuterated solvents. High Resolution Mass Spectra (HRMS) were obtained with an Agilent LCTOF 6200 series mass spectrometer using electrospray ionization (ESI) and APPI.

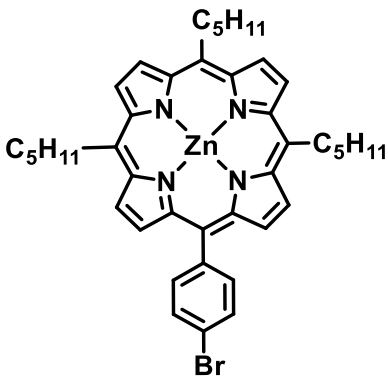

Compound A

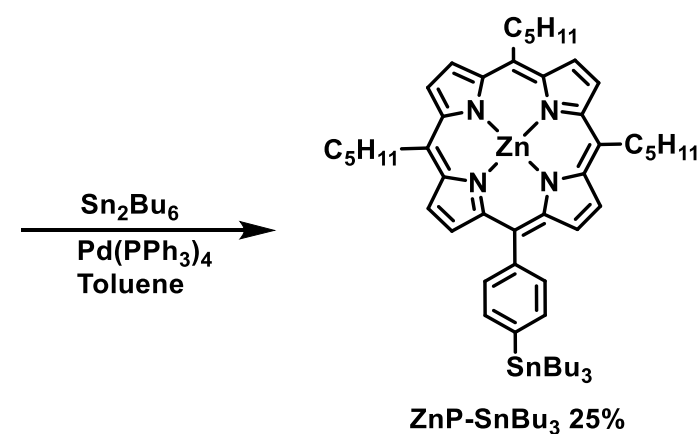

$\mathrm{ZnP}-\mathrm{SnBu}_{3}$ 25\%

ZnP-SnBu $3.158 \mathrm{mg}$ of Compound A $(0.21 \mathrm{mmol}), 600 \mathrm{mg}$ of hexabutylditin $(1.05 \mathrm{mmol})$ were combined in $10 \mathrm{~mL}$ anhydrous toluene and this solution was purged with nitrogen for $15 \mathrm{~min}$. After adding $10 \mathrm{mg}$ of tetrakis(triphenylphosphine)palladium(0) $(0.01 \mathrm{mmol})$ to the solution, the solution was purged with nitrogen for another $15 \mathrm{~min}$. The solution was then heated at $110^{\circ} \mathrm{C}$ for $12 \mathrm{~h}$. The reaction mixture was cooled and then the solvent was then removed by rotary evaporation. The crude product was quickly run through a $5 \mathrm{~cm}$ neutral alumina plug with hexanes/DCM (90/10) as the mobile phase, yielding $\mathbf{Z n P - S n B u}$ (50 mg, 25\%). The product was directly used for next step without further purification due to lack of stability. 


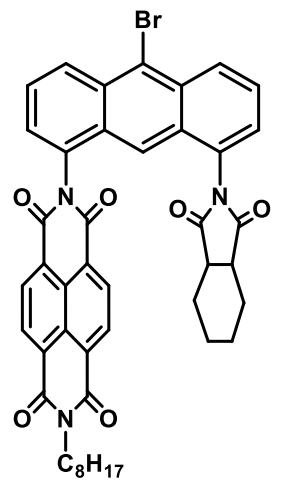

Compound B

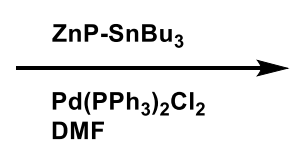

DMF

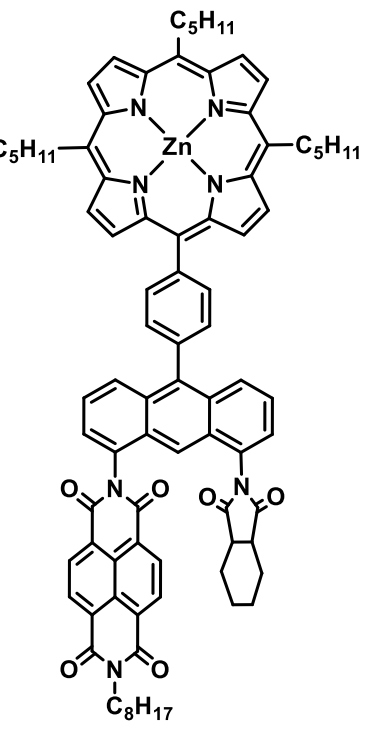

ZnP-An-NDI (1) $25 \%$

ZnP-An-NDI (1). $36 \mathrm{mg}$ of $\mathbf{Z n P - S n B u} 3$ (0.038 mmol), $20 \mathrm{mg}$ of Compound B (0.025 mmol) were combined in $10 \mathrm{~mL}$ anhydrous DMF and this solution was purged with nitrogen for 15 min. After adding $3 \mathrm{mg}$ of Bis(triphenylphosphine)palladium(II) dichloride (0.004 mmol) to the solution, the solution was purged with nitrogen for another $15 \mathrm{~min}$. The solution was then heated at $80^{\circ} \mathrm{C}$ for $16 \mathrm{~h}$. The reaction mixture was cooled and then the solvent was then removed by rotary evaporation. The crude product was first purified by silica gel column chromatography with hexanes/ethyl acetate (70/30) as the mobile phase. The resulting product was again purified by neutral alumina column chromatography with DCM/ethyl acetate (90/10) as the mobile phase yielding 1 (13 mg, 25\%). ${ }^{1} \mathrm{H}$ NMR (500 MHz, $\left.\mathrm{CDCl}_{3}\right): \delta 9.64(\mathrm{~s}, 6 \mathrm{H}), 9.20-9.16(\mathrm{~m}, 4 \mathrm{H}), 8.91$ $(\mathrm{d}, J=7.5 \mathrm{~Hz}, 2 \mathrm{H}), 8.86(\mathrm{~d}, J=7.5 \mathrm{~Hz}, 2 \mathrm{H}), 8.46(\mathrm{~d}, J=7.5 \mathrm{~Hz}, 2 \mathrm{H}), 8.37(\mathrm{~d}, J=8.5 \mathrm{~Hz}, 1 \mathrm{H})$, $8.29(\mathrm{~d}, J=8.5 \mathrm{~Hz}, 1 \mathrm{H}), 8.13(\mathrm{~s}, 1 \mathrm{H}), 7.88(\mathrm{~d}, J=7.5 \mathrm{~Hz}, 2 \mathrm{H}), 7.81-7.75(\mathrm{~m}, 2 \mathrm{H}), 7.68-7.62(\mathrm{~m}$, 2H), $7.39(\mathrm{~d}, J=7.5 \mathrm{~Hz}, 2 \mathrm{H}), 5.07-5.02(\mathrm{~m}, 5 \mathrm{H}), 4.31-4.27(\mathrm{~m}, 3 \mathrm{H}), 3.71-3.68(\mathrm{~m}, 2 \mathrm{H}), 2.92-$ $2.88(\mathrm{~m}, 1 \mathrm{H}), 2.63-2.58(\mathrm{~m}, 5 \mathrm{H}), 1.91-1.78(\mathrm{~m}, 9 \mathrm{H}), 1.65-1.55(\mathrm{~m}, 7 \mathrm{H}), 1.45-1.25(\mathrm{~m}, 13 \mathrm{H}) 1.05-$ $1.01(\mathrm{~m}, 8 \mathrm{H}), 0.93-0.88(\mathrm{~m}, 4 \mathrm{H}) .{ }^{13} \mathrm{C}$ NMR was not obtained due to aggregation in highly 
concentrated solution. HRMS (m/z) for $\mathrm{C}_{85} \mathrm{H}_{83} \mathrm{~N}_{7} \mathrm{O}_{6} \mathrm{Zn}$ calculated: 1361.56963, found: 1361.57197.

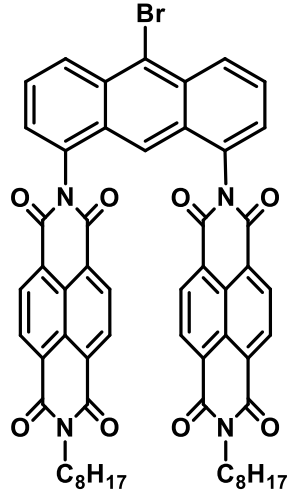

Compound C

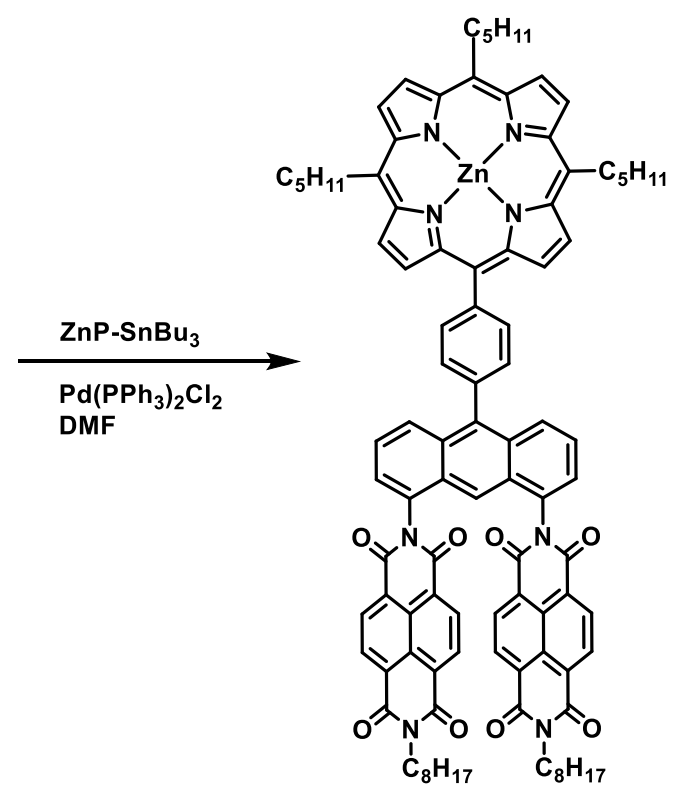

ZnP-An-NDI 2 (2) $30 \%$

ZnP-An-NDI $\mathbf{2}_{2}$ (2). $36 \mathrm{mg}$ of $\mathbf{Z n P - S n B u} 3$ (0.038 mmol), $25 \mathrm{mg}$ of Compound C (0.025 mmol) were combined in $10 \mathrm{~mL}$ anhydrous DMF and this solution was purged with nitrogen for $15 \mathrm{~min}$. After adding $3 \mathrm{mg}$ of Bis(triphenylphosphine)palladium(II) dichloride (0.004 mmol) to the solution, the solution was purged with nitrogen for another $15 \mathrm{~min}$. The solution was then heated at $80^{\circ} \mathrm{C}$ for $16 \mathrm{~h}$. The reaction mixture was cooled and then the solvent was then removed by rotary evaporation. The crude product was first purified by silica gel column chromatography with hexanes/ethyl acetate (70/30) as the mobile phase. The resulting product was again purified by neutral alumina column chromatography with DCM/ethyl acetate (90/10) as the mobile phase yielding 2 (17 mg, 30\%). ${ }^{1} \mathrm{H}$ NMR (500 $\left.\mathrm{MHz} \mathrm{CDCl}_{3}\right): \delta 9.67(\mathrm{~s}, 4 \mathrm{H}), 9.65(\mathrm{~d}, J=5.0 \mathrm{~Hz}, 2 \mathrm{H})$, $9.20(\mathrm{~d}, J=5.0 \mathrm{~Hz}, 2 \mathrm{H}), 8.45-8.40(\mathrm{~m}, 10 \mathrm{H}), 8.37(\mathrm{~d}, J=9.0 \mathrm{~Hz}, 1 \mathrm{H}), 7.93(\mathrm{~d}, J=8.0 \mathrm{~Hz}, 2 \mathrm{H})$, $7.86(\mathrm{~s}, 1 \mathrm{H}), 7.77(\mathrm{dd}, J=7.0 \mathrm{~Hz}, J=8.0 \mathrm{~Hz}, 2 \mathrm{H}), 7.81-7.75(\mathrm{~m}, 2 \mathrm{H}), 7.63(\mathrm{~d}, J=9.0 \mathrm{~Hz}, 1 \mathrm{H})$, 5.06-5.02 (m, 5H), 4.29-4.24 (m, 3H), 2.68-2.60 (m, 4H), 1.91-1.84 (m, 4H), 1.82-1.75 (m, 3H), 
1.65-1.58 (m, 5H), 1.54-1.20 (m, 20H), 1.06-1.01 (m, 8H), 0.93-0.88 (m, 6H). ${ }^{13} \mathrm{C}$ NMR was not obtained due to aggregation in highly concentrated solution. HRMS (m/z) for $\mathrm{C}_{99} \mathrm{H}_{94} \mathrm{~N}_{8} \mathrm{O}_{8} \mathrm{Zn}$ calculated: 1586.64861 , found: 1586.64549 . 


\section{Visible and NIR Transient Absorption Spectroscopy}

\subsection{Toluene Data}

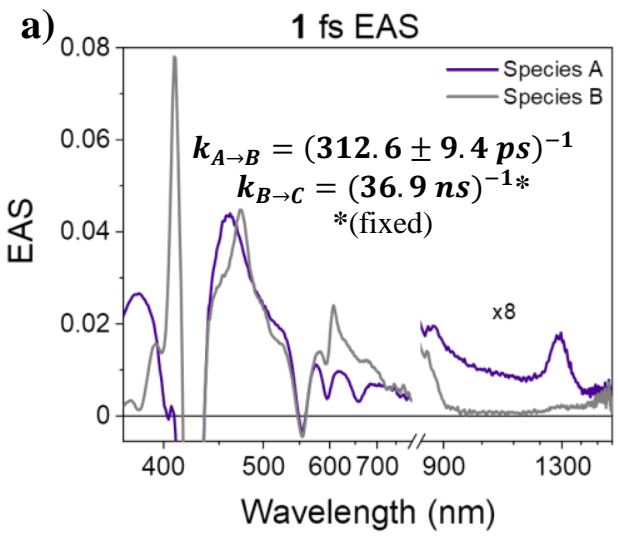

b)
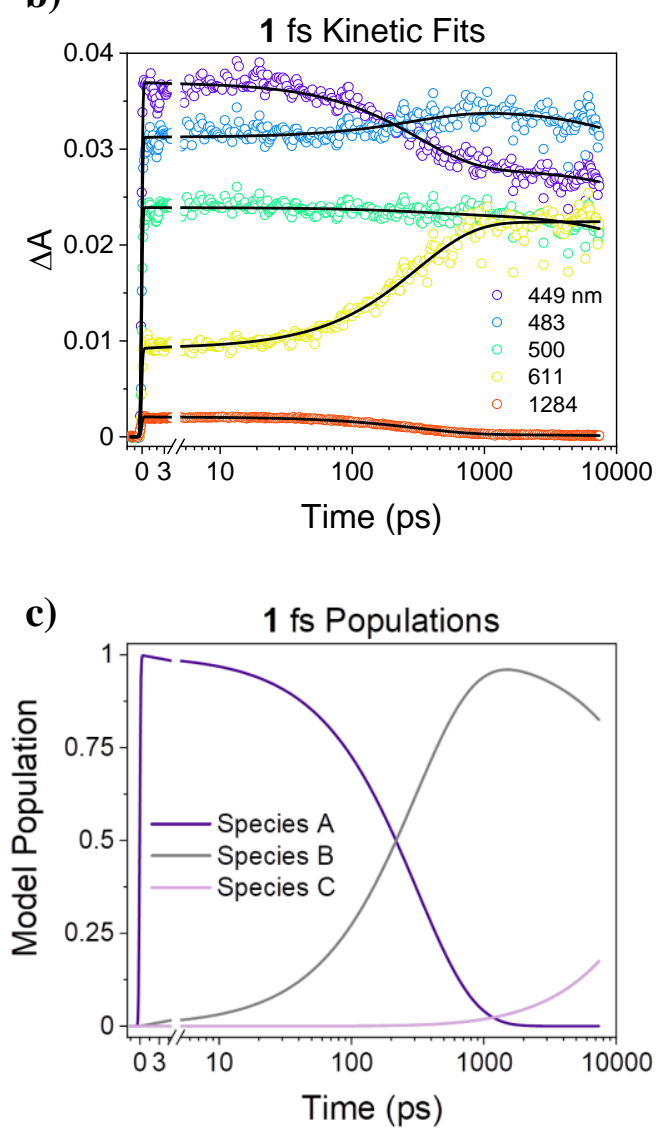

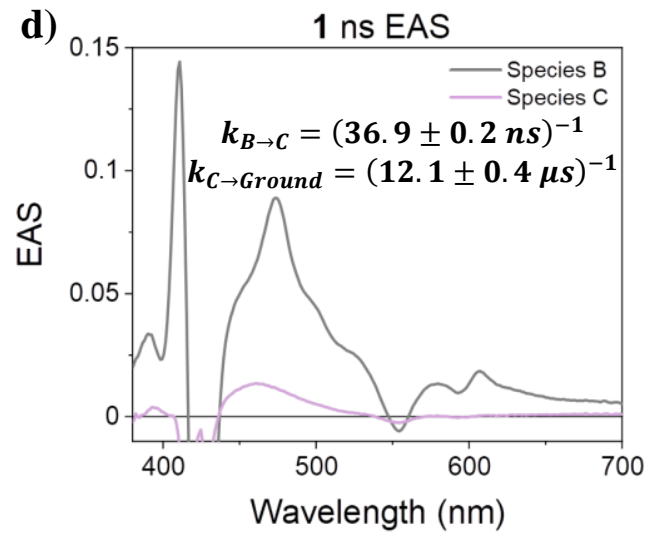

e)
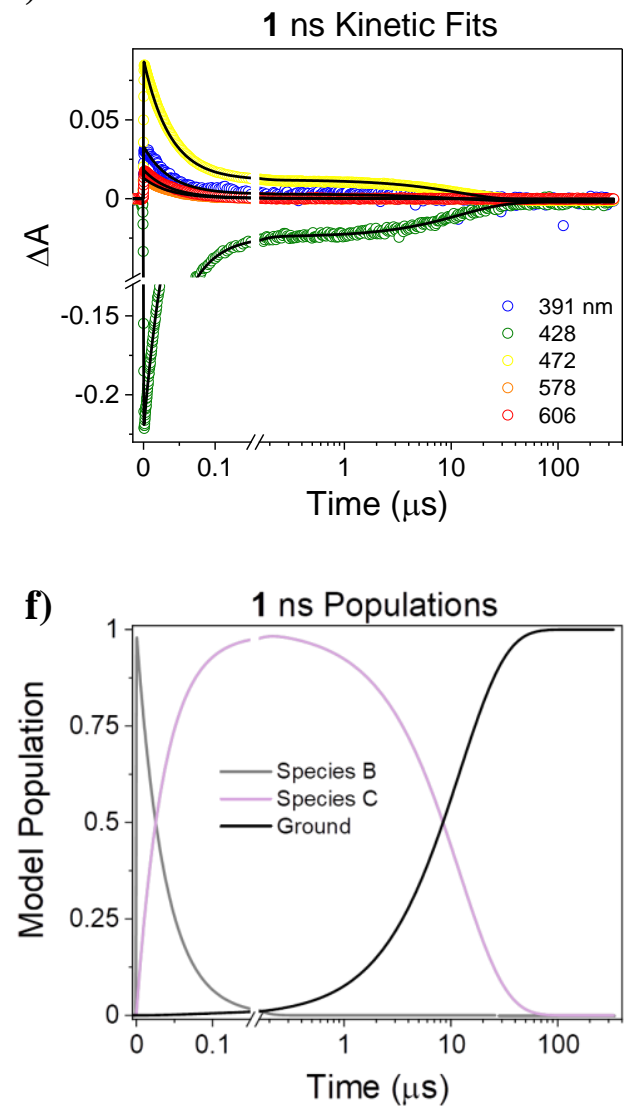

Figure S1. Kinetic analysis of the femtosecond (a-c) and nanosecond (d-f) transient absorption data for 1 photo excited at $414 \mathrm{~nm}$ in room temperature toluene. Femtosecond data were fit to an $\mathrm{A} \rightarrow \mathrm{B} \rightarrow \mathrm{C}$ kinetic model with the $\mathrm{B} \rightarrow \mathrm{C}$ rate constant held fixed from the corresponding nanosecond data fit. Nanosecond data were fit to a $\mathrm{B} \rightarrow \mathrm{C} \rightarrow$ Ground kinetic model. (a and d) Evolution-associated spectra (EAS) with rates. Uncertainties are errors of the fits. ( $b$ and e) Kinetic time traces and their associated fits. (c and f) Population models for the species in the EAS. 

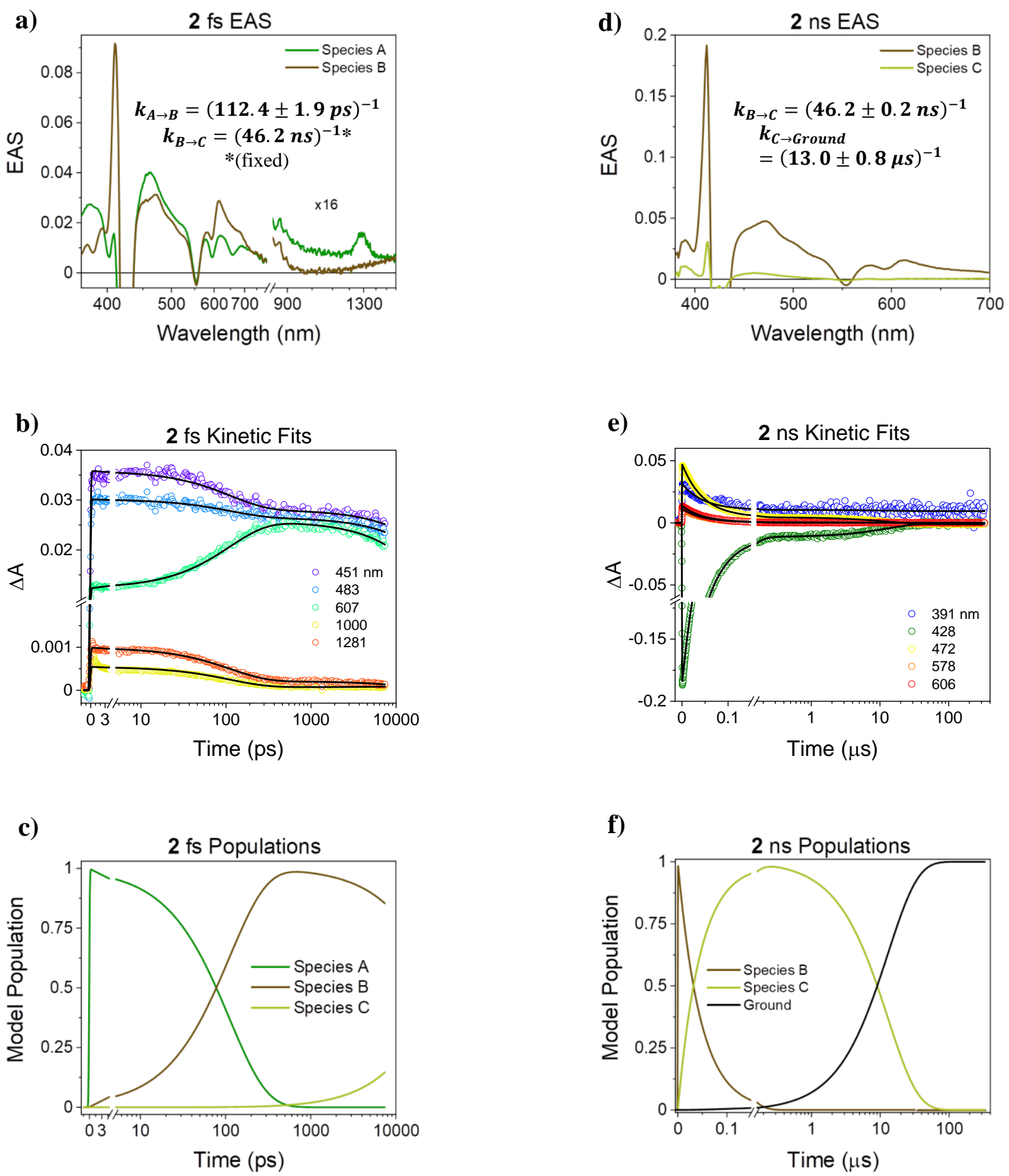

Figure S2. Kinetic analysis of the femtosecond (a-c) and nanosecond (d-f) transient absorption data for 2 photo excited at $414 \mathrm{~nm}$ in room temperature toluene. Femtosecond data were fit to an $\mathrm{A} \rightarrow \mathrm{B} \rightarrow \mathrm{C}$ kinetic model with the $\mathrm{B} \rightarrow \mathrm{C}$ rate constant held fixed from the corresponding nanosecond data fit. Nanosecond data were fit to a $\mathrm{B} \rightarrow \mathrm{C} \rightarrow$ Ground kinetic model. (a and d) Evolution-associated spectra (EAS) with rates. Uncertainties are errors of the fits. (b and e) Kinetic time traces and their associated fits. (c and f) Population models for the species in the EAS. 
a)

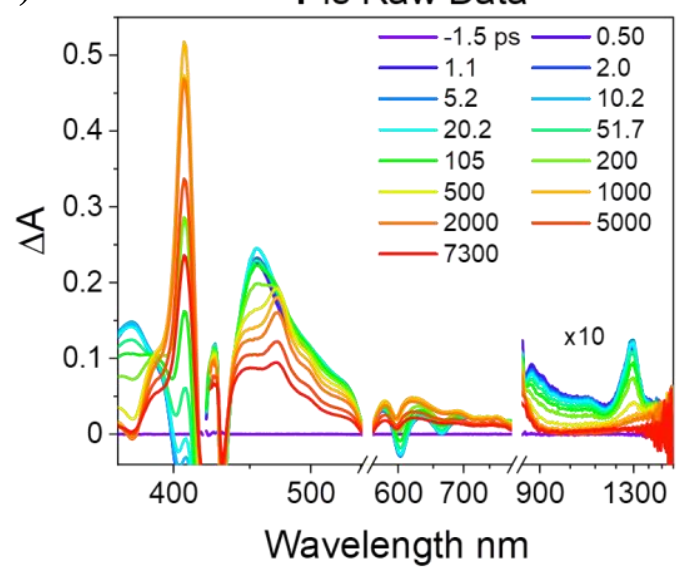

c)

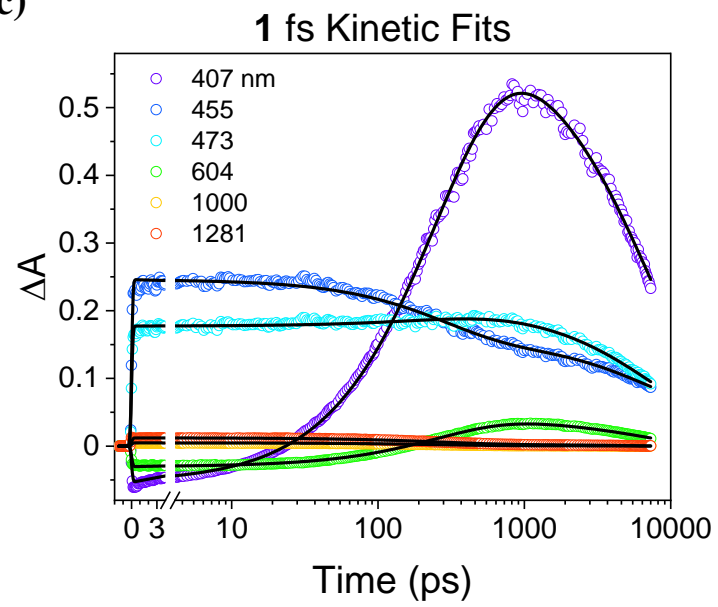

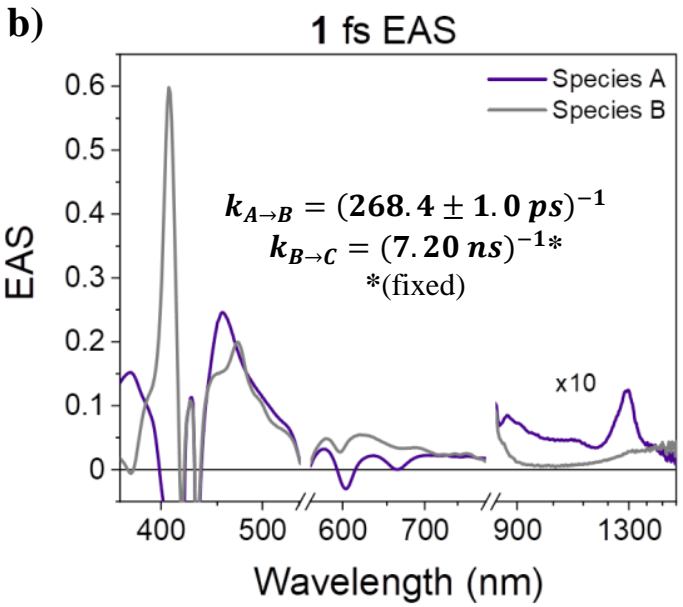

d)

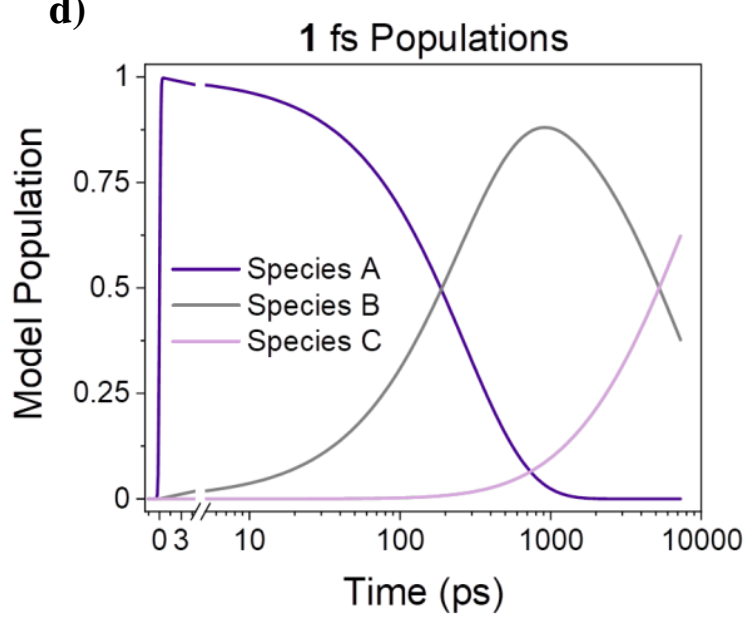

Figure S3. Kinetic analysis of the femtosecond transient absorption data for $\mathbf{1}$ photo excited at $560 \mathrm{~nm}$ in room temperature 1,4-dioxane- $\mathrm{d}_{8}$. Femtosecond data were fit to an $\mathrm{A} \rightarrow \mathrm{B} \rightarrow \mathrm{C}$ kinetic model with the $\mathrm{B} \rightarrow \mathrm{C}$ rate constant held fixed from the corresponding nanosecond data fit (Figure S4). (a) Raw data with select spectral traces. Spectral features appear at approximately the same wavelengths as described in the toluene data in the main text. (b) Evolution-associated spectra (EAS) with rates. Uncertainties are errors of the fits. Particular species and associated rates are described by the same physical phenomena as outlined in the toluene data in the main text. (c) Kinetic time traces and their associated fits. (d) Population models for the species in the EAS. 
a)

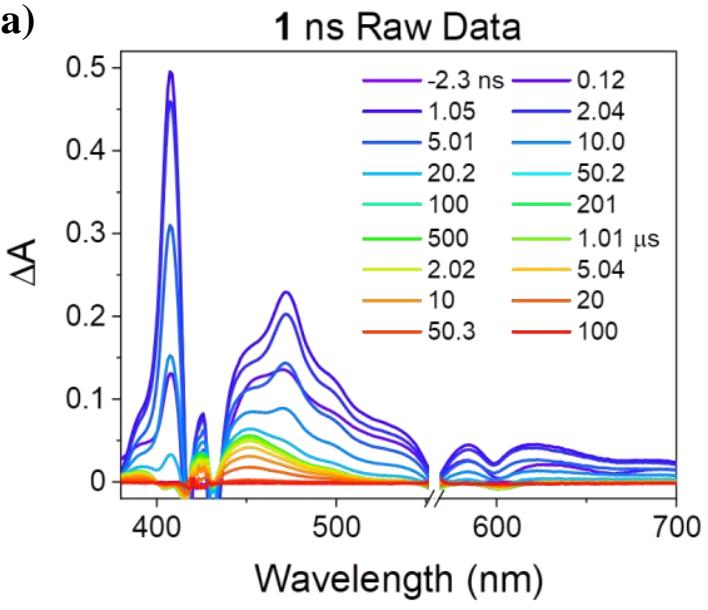

c)

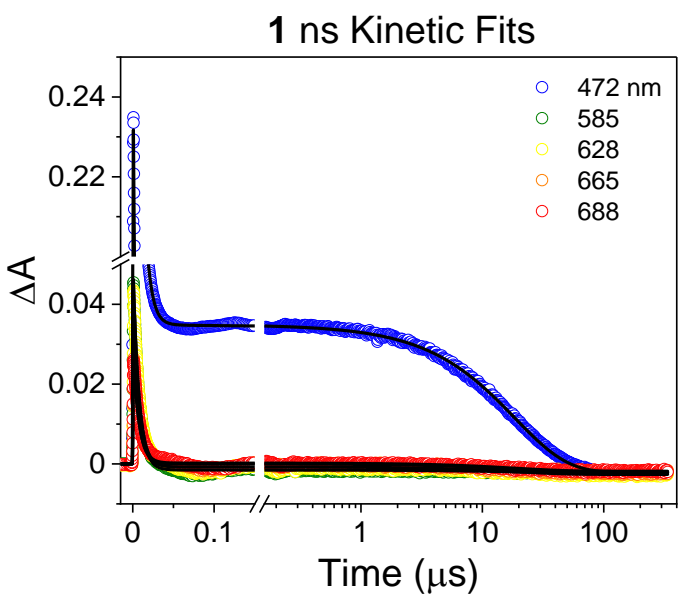

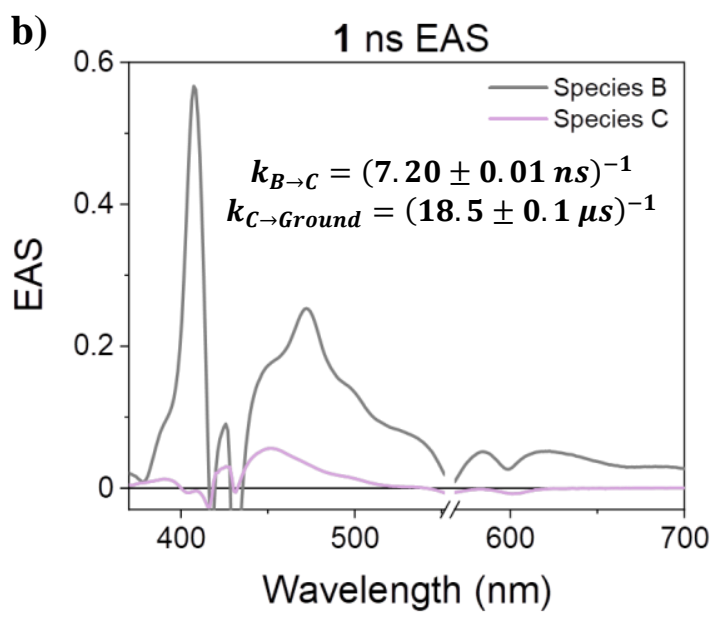

d)

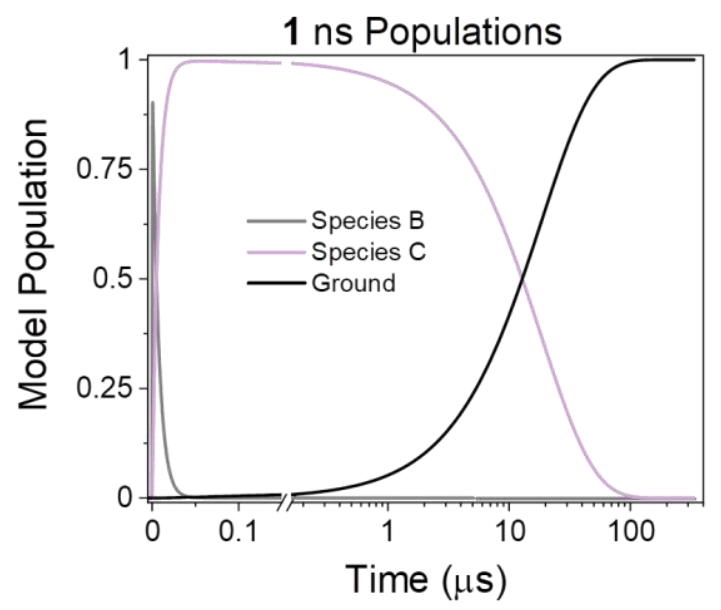

Figure S4. Kinetic analysis of the nanosecond transient absorption data for $\mathbf{1}$ photo excited at $560 \mathrm{~nm}$ in room temperature $1,4-$ dioxane- $\mathrm{d}_{8}$. Nanosecond data were fit to a $\mathrm{B} \rightarrow \mathrm{C} \rightarrow$ Ground kinetic model. (a) Raw data with select spectral traces. Spectral features appear at approximately the same wavelengths as described in the toluene data in the main text. (b) Evolution-associated spectra (EAS) with rates. Uncertainties are errors of the fits. Particular species and associated rates are described by the same physical phenomena as outlined in the toluene data in the main text. (c) Kinetic time traces and their associated fits. (d) Population models for the species in the EAS. 
a)

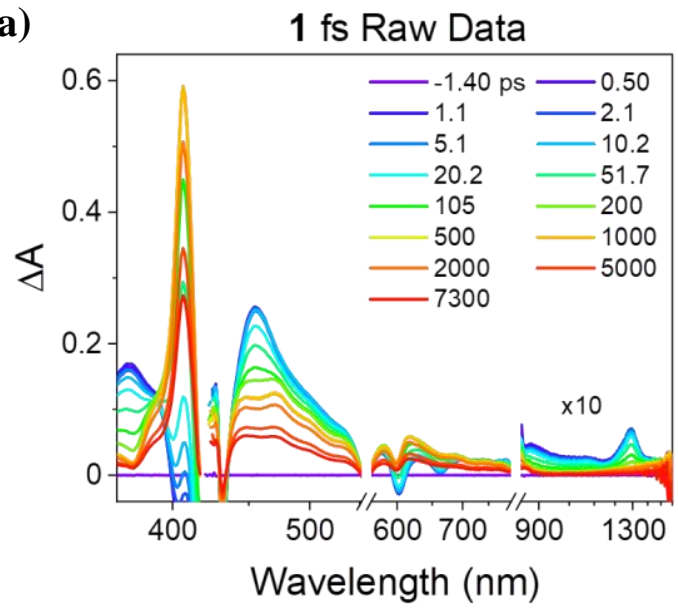

c)

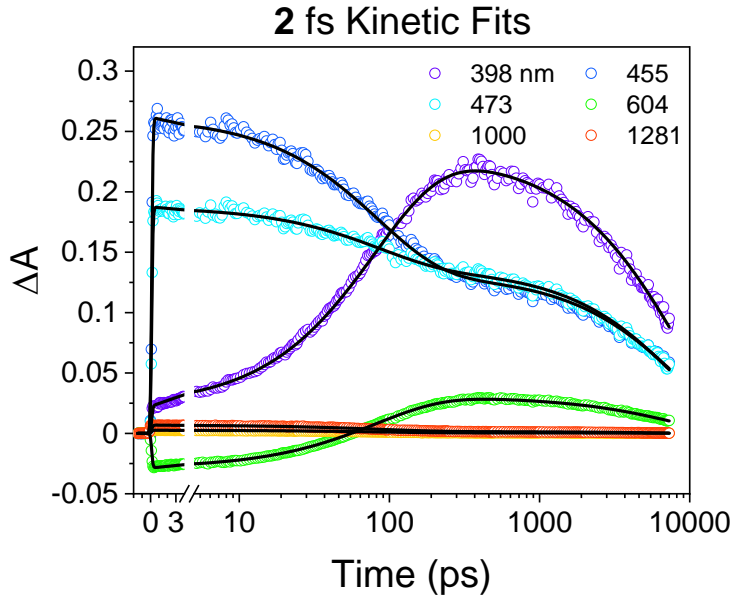

b)

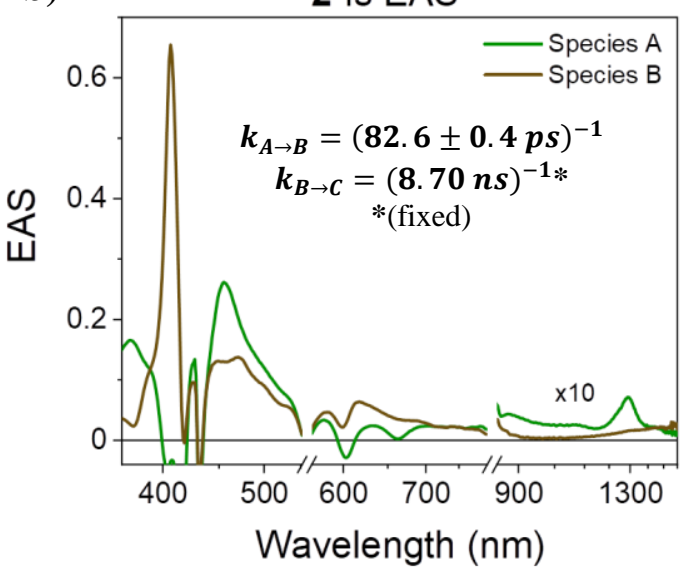

d)

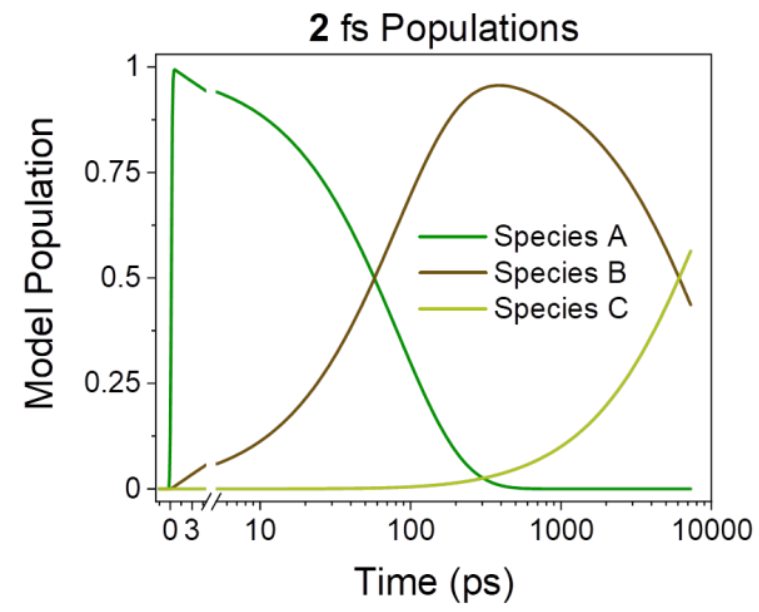

Figure S5. Kinetic analysis of the femtosecond transient absorption data for $\mathbf{2}$ photo excited at $560 \mathrm{~nm}$ in room temperature 1,4 -dioxane- $\mathrm{d}_{8}$. Femtosecond data were fit to an $\mathrm{A} \rightarrow \mathrm{B} \rightarrow \mathrm{C}$ kinetic model with the $\mathrm{B} \rightarrow \mathrm{C}$ rate constant held fixed from the corresponding nanosecond data fit (Figure S6). (a) Raw data with select spectral traces. Spectral features appear at approximately the same wavelengths as described in the toluene data in the main text. (b) Evolution-associated spectra (EAS) with rates. Uncertainties are errors of the fits. Particular species and associated rates are described by the same physical phenomena as outlined in the toluene data in the main text. (c) Kinetic time traces and their associated fits. (d) Population models for the species in the EAS. 
a)

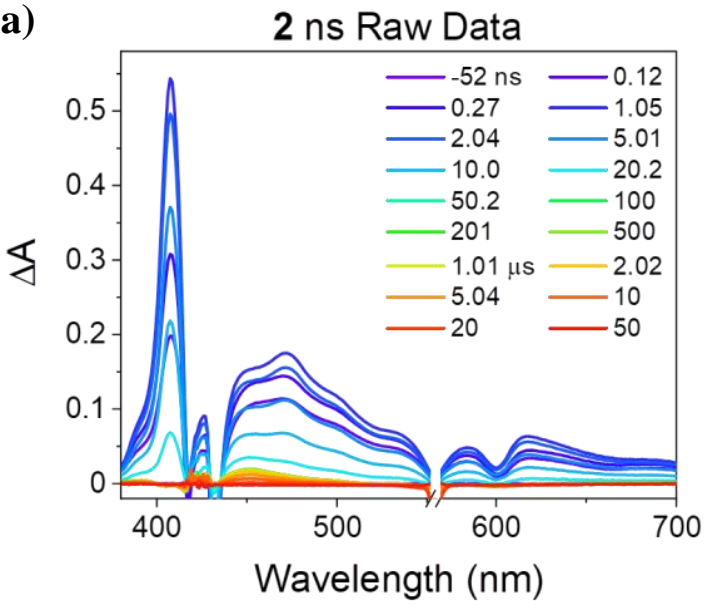

c)

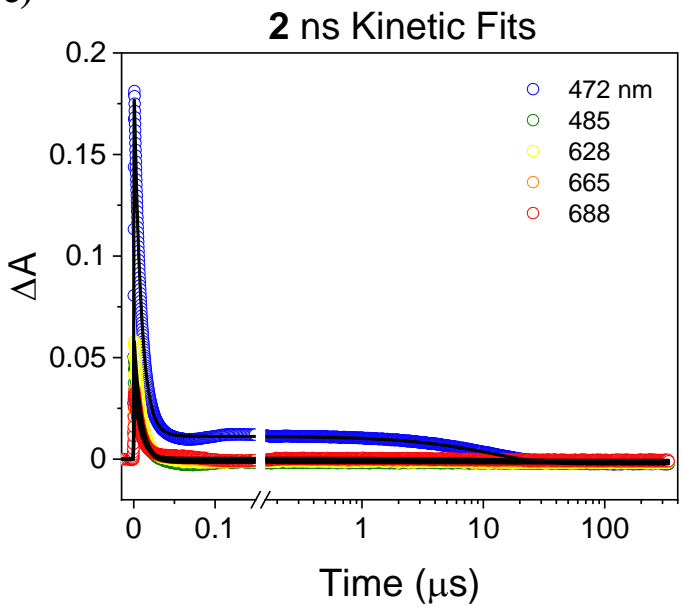

b)

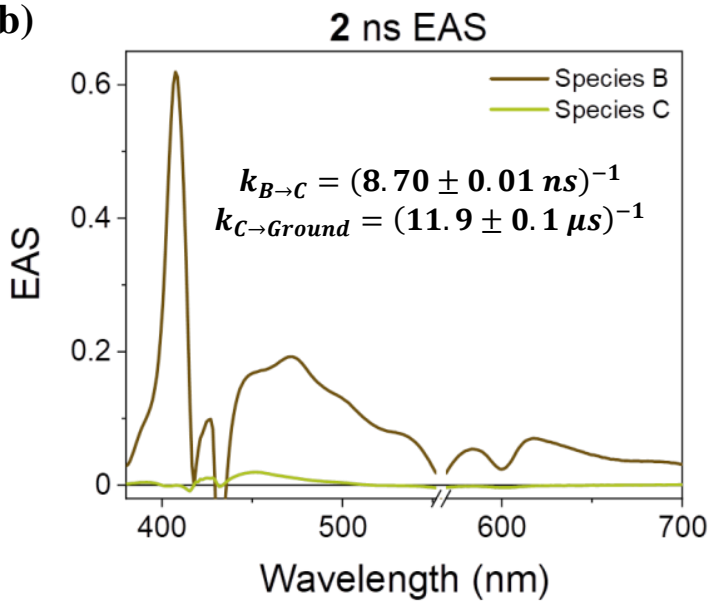

d)

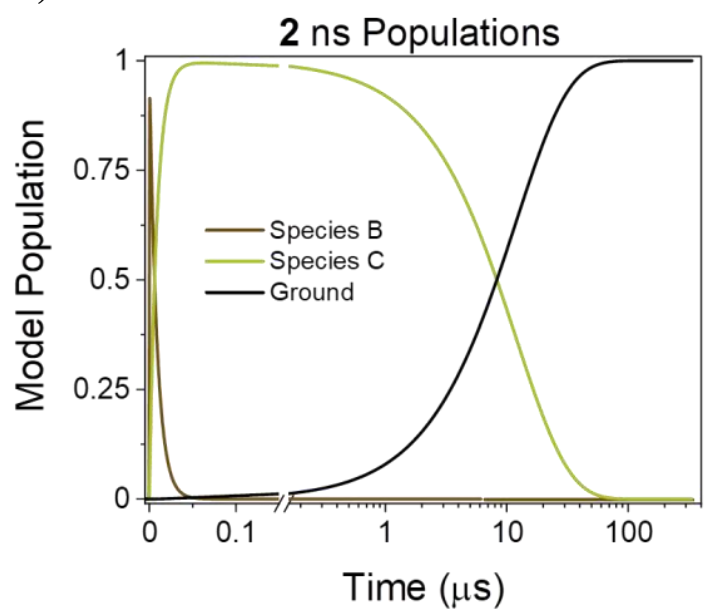

Figure S6. Kinetic analysis of the nanosecond transient absorption data for $\mathbf{2}$ photoexcited at $560 \mathrm{~nm}$ in room temperature 1,4-dioxane- $\mathrm{d}_{8}$. Nanosecond data were fit to a B $\rightarrow \mathrm{C} \rightarrow$ Ground kinetic model. (a) Raw data with select spectral traces. Spectral features appear at approximately the same wavelengths as described in the toluene data in the main text. (b) Evolution-associated spectra (EAS) with rates. Uncertainties are errors of the fits. Particular species and associated rates are described by the same physical phenomena as outlined in the toluene data in the main text. (c) Kinetic time traces and their associated fits. (d) Population models for the species in the EAS. 


\section{Transient Femtosecond IR Spectroscopy}

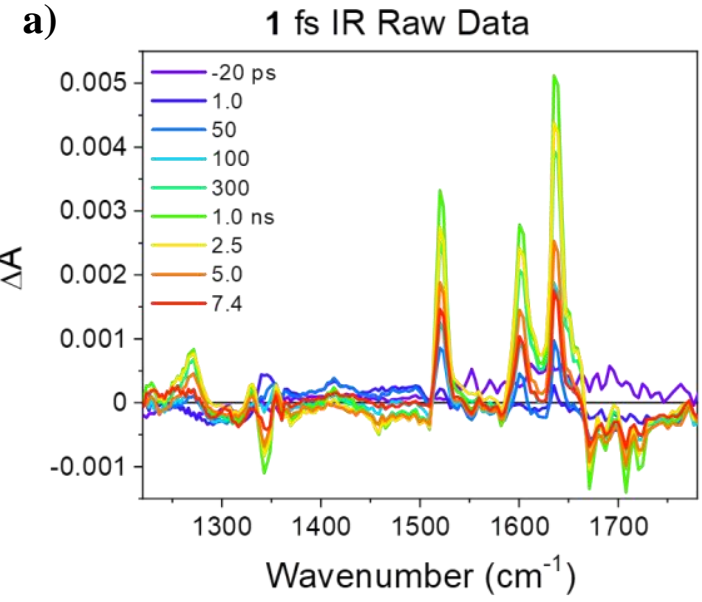

c)

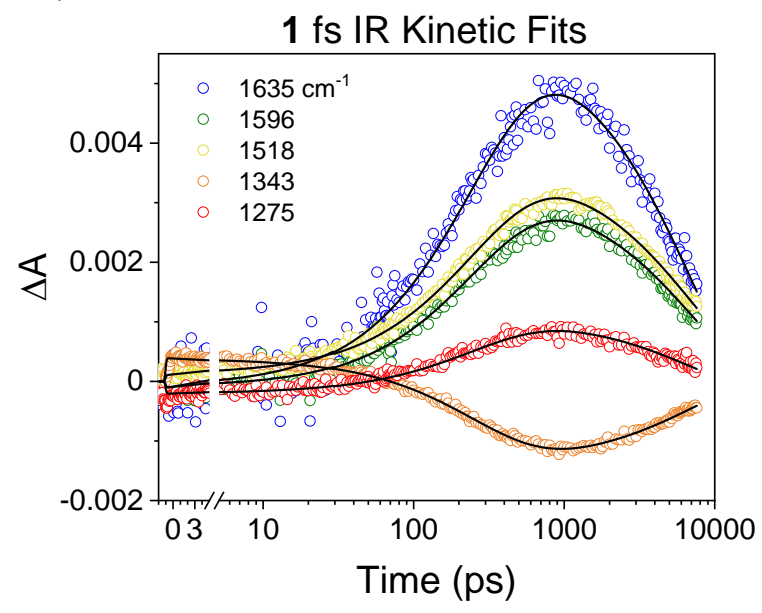

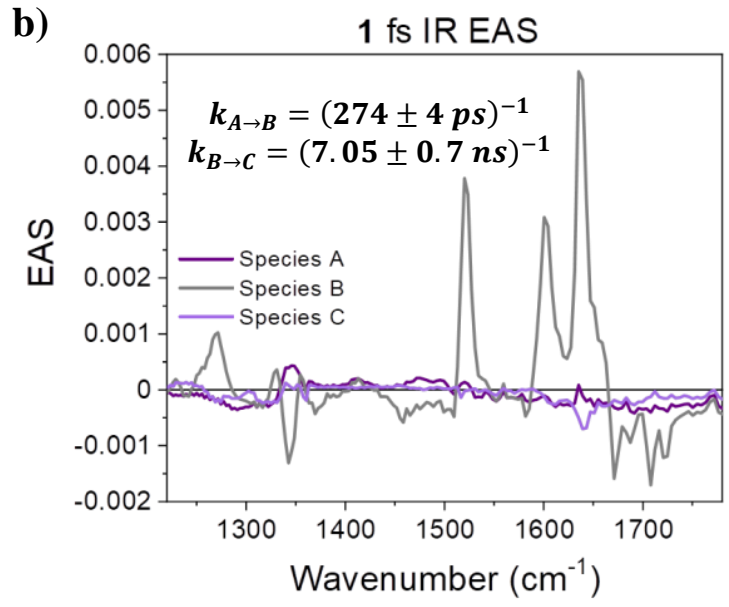

d)

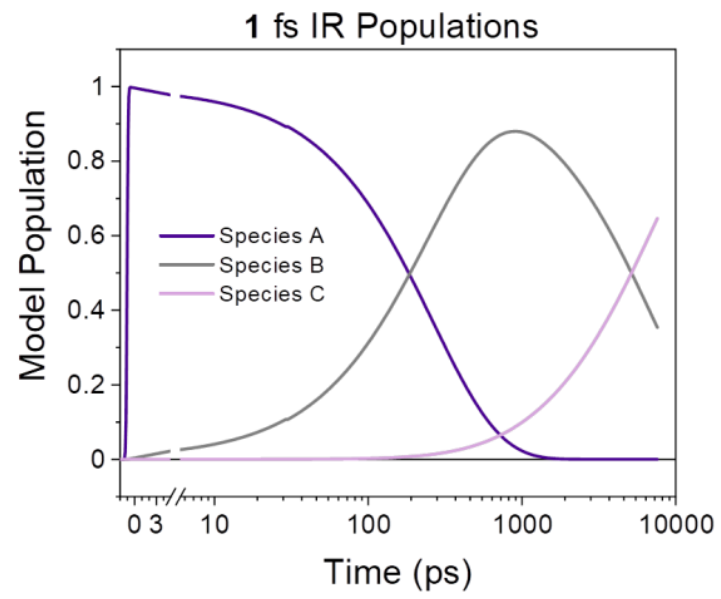

Figure S7. Kinetic analysis of the corrected (see Figure S9) femtosecond infrared transient absorption data for 1 photo excited at $560 \mathrm{~nm}$ in room temperature 1,4-dioxane- $\mathrm{d}_{8}$. Femtosecond data were fit to an $\mathrm{A} \rightarrow \mathrm{B} \rightarrow \mathrm{C}$ kinetic model. (a) Raw data with select spectral traces. (b) Evolution-associated spectra (EAS) with rates. Uncertainties are errors of the fits. Particular species and associated rates are described by the same physical phenomena as outlined in the toluene data in the main text. (c) Kinetic time traces and their associated fits. (d) Population models for the species in the EAS. See section 8.3 for mode assignments. 
a)

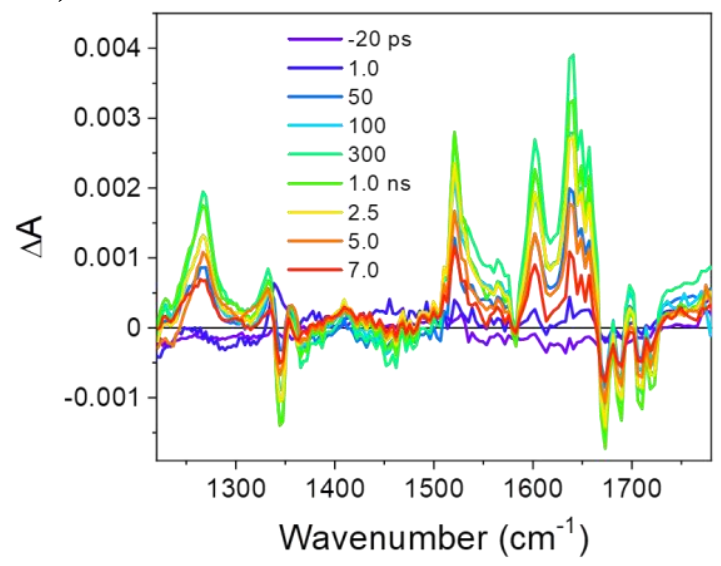

c)

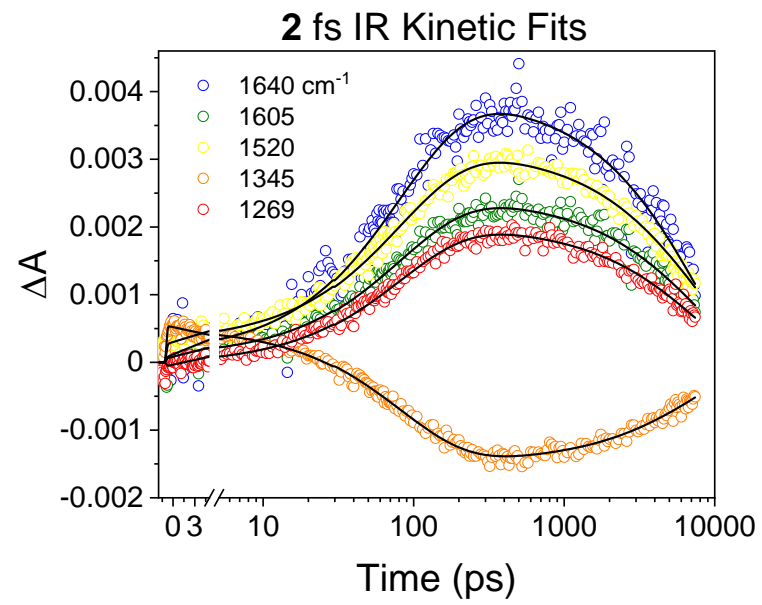

b)

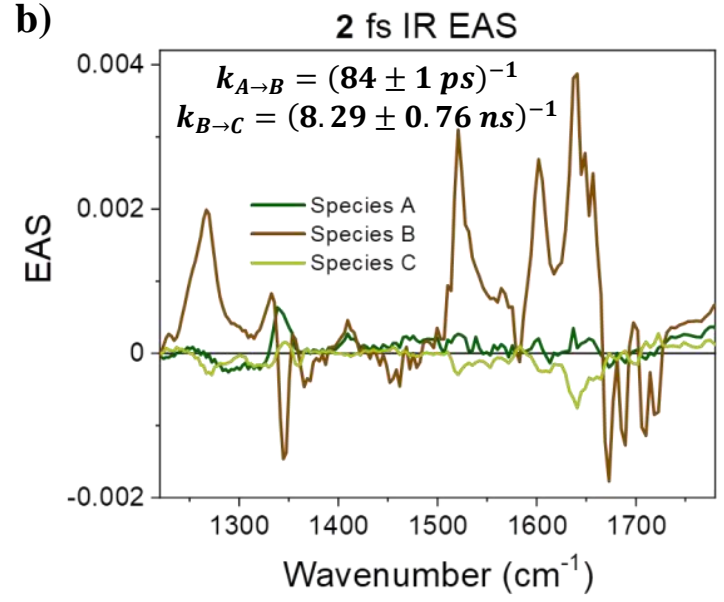

d)

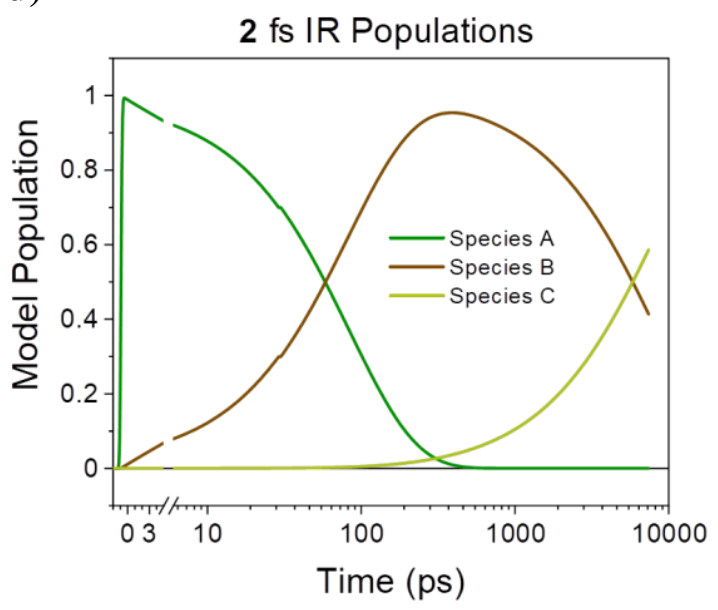

Figure S8. Kinetic analysis of the corrected (see Figure S9) femtosecond infrared transient absorption data for 2 photo excited at $560 \mathrm{~nm}$ in room temperature 1,4-dioxane- $\mathrm{d}_{8}$. Femtosecond data were fit to an $\mathbf{A} \rightarrow \mathbf{B} \rightarrow \mathbf{C}$ kinetic model. (a) Raw data with select spectral traces. (b) Evolution-associated spectra (EAS) with rates. Uncertainties are errors of the fits. Particular species and associated rates are described by the same physical phenomena as outlined in the toluene data in the main text. (c) Kinetic time traces and their associated fits. (d) Population models for the species in the EAS. See section 8.3 for mode assignments. 


\section{Fourier-Transform IR (FT-IR) Spectroscopy}

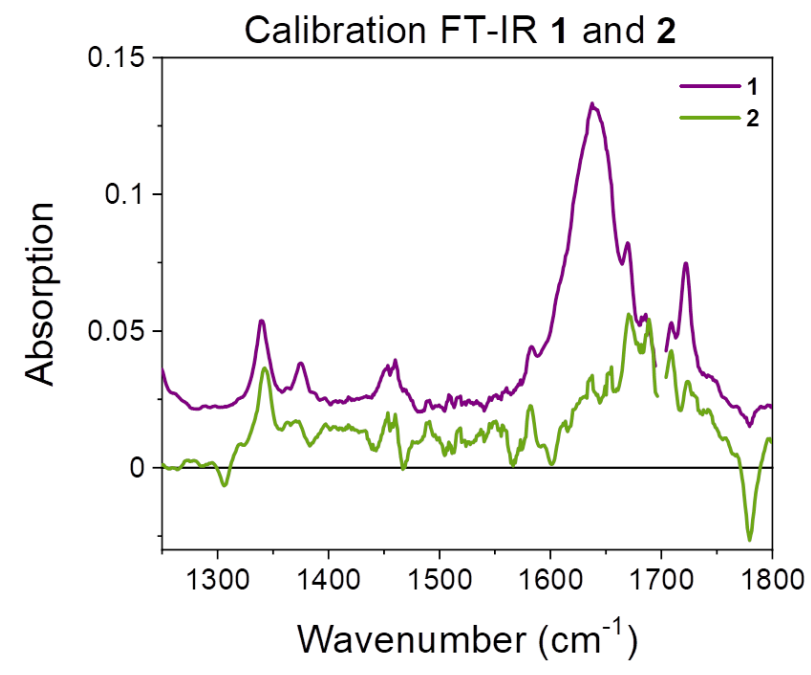

Figure S9. FT-IR steady-state spectroscopy data for $\mathbf{1}$ and $\mathbf{2}$. These data were collected in 1,4dioxane- $\mathrm{d}_{8}$ with an optical density of $\sim 0.125$ at $560 \mathrm{~nm}$ with a $500 \mu \mathrm{m}$ path length. The fs IR data was calibrated by correlating the wavenumber of certain features in these data with those in the fs IR data. Then a quadratic relationship was established between the two data sets to correct the frequency-axes of the fs IR data. See section 8.3 for mode assignments. 


\section{Calculation of Free Energies of Reaction and Reorganization Energies}

The free energy of charge separation $\Delta G_{C S}$ was computed using the formulation of Weller: ${ }^{3}$

$$
\Delta G_{C S}=\Delta G_{I P}-E_{00}=e\left(E_{D}-E_{A}\right)-\frac{e^{2}}{r_{D A} \varepsilon_{S}}+e^{2}\left(\frac{1}{2 r_{D}}+\frac{1}{2 r_{A}}\right)\left(\frac{1}{\varepsilon_{S}}-\frac{1}{\varepsilon_{S p}}\right)-E_{00}
$$

where $\Delta G_{I P}$ is the free energy of the radical ion pair, $E_{00}$ is the energy of the donor singlet excited state, and $\varepsilon_{s p}$ is the dielectric constant of the polar solvent used in the electrochemistry experiments for the donor and acceptor $e$ is the charge of the electron; $r_{D}$ and $r_{A}$ are the radii of the donor and acceptor radical ions, respectively, and $r_{D A}$ is the center-to-center distance between the radical ions. The one-electron oxidation potential of $\mathrm{ZnP}$ is $E_{D}=0.65 \mathrm{~V}$ vs. SCE in butyronitrile, ${ }^{4}$ while the one-electron reduction potentials $E_{A}=-0.54 \mathrm{~V}$ for NDI and $-0.45 \mathrm{~V}$ for $\mathrm{NDI}_{2}$ (both vs. SCE) in dichloromethane. ${ }^{2}$ For $\mathbf{1}$ and $\mathbf{2}, r_{D}=5.0 \AA r_{A}=4.3 \AA$ and $r_{D A}=17.2 \AA$, were determined using DFT geometry-optimized structures. Using $E_{00}=2.06 \mathrm{eV},{ }^{5}$ we calculated $\Delta G_{C S}=-0.27$ and $-0.36 \mathrm{eV}$ for $\mathbf{1}$ and $\mathbf{2}$, respectively.

The total reorganization energy for charge separation (and recombination) is the sum of the internal $\left(\lambda_{i}\right)$ and solvent $\left(\lambda_{s}\right)$ reorganization energies: $\lambda=\lambda_{i}+\lambda_{s}$. The internal reorganization energies $\lambda_{i}$ were determined via density functional theory (DFT) computations (see section 8 below), giving $\lambda_{i}=0.24 \mathrm{eV}$ for $\mathbf{1}$ and $0.27 \mathrm{eV}$ for $\mathbf{2}$. The solvent reorganization energy is calculated based on the dielectric continuum model: ${ }^{6}$

$$
\lambda_{s}=e^{2}\left(\frac{1}{2 r_{D}}+\frac{1}{2 r_{A}}-\frac{1}{r_{D A}}\right)\left(\frac{1}{\varepsilon_{o p}}-\frac{1}{\varepsilon_{S}}\right)
$$

where $\varepsilon_{o p}$ is the high-frequency dielectric constant of the solvent, which is approximately equal to the square of the refractive index ( $\varepsilon_{o p}=2.25$ for toluene) and $\varepsilon_{s}$ is the dielectric constant of the solvent ( $\varepsilon_{o p}=2.38$ for toluene). Using the values of $r_{D}, r_{A}$, and $r_{D A}$ given above, $\lambda_{s}=0.05 \mathrm{eV}$ 
for both $\mathbf{1}$ and 2. Thus, the total reorganization energies are $0.29 \mathrm{eV}$ and $0.32 \mathrm{eV}$ for $\mathbf{1}$ and $\mathbf{2}$, respectively.

\section{Estimation of Observed ${ }^{3^{*}} \mathrm{ZnP}$ Yield in $295 \mathrm{~K}$ Toluene}

Using the EAS of both $\mathbf{1}$ and $\mathbf{2}$ on the nanosecond to microsecond time scale shown in Figures $\mathrm{S} 4 \mathrm{~b}$ and S6b, respectively, the initial bleach of the $\mathrm{Q}$ band at $555 \mathrm{~nm}\left(\Delta A_{555}\right)$ was used to determine the initial amount of $\mathrm{ZnP}$ excited and the absorption band at $458 \mathrm{~nm}$ was used to determine the amount of ${ }^{3 *} \mathrm{ZnP}$ formed. The $\mathrm{ZnP}$ Q-band at $555 \mathrm{~nm}$ has an extinction coefficient $\epsilon_{555}=2.0 \times 10^{4} \mathrm{M}^{-1} \mathrm{~cm}^{-1},{ }^{7}$ while the extinction coefficient of ${ }^{3 *} \mathrm{ZnP}$ at $458 \mathrm{~nm}$ is $8 \times 10^{3} \mathrm{M}^{-1} \mathrm{~cm}^{-}$ ${ }^{18-9}$ The yield of ${ }^{3 *} \mathrm{ZnP}$ produced by charge recombination was calculated using the following expression:

$$
\phi_{T}=\frac{\Delta A_{458} \cdot \epsilon_{555}}{\Delta A_{555} \cdot \epsilon_{458}}
$$

\section{Magnetic Field Effect Transient Absorption Experiments}

Estimation of mean hyperfine fields was performed using the previously-described model ${ }^{10}$ which includes the following equations:

$$
B_{h f i}=\sqrt{3\left(B_{1}^{2}+B_{2}^{2}\right)}
$$

Each $B_{i}$ is the hyperfine field of a particular radical species given by

$$
B_{i}=\sqrt{\sum_{k} a_{i k}^{2} I_{i k}\left(I_{i k}+1\right)}
$$

with $a_{i k}$ being the isotropic hyperfine coupling constant of a certain nucleus $k$ as part of a certain radical $i$ and $I_{i k}$ being the nuclear spin quantum number of the nucleus $k$ interacting with radical $i$. Using previously-reported values, ${ }^{11-12}$ the hyperfine fields for the $\mathrm{ZnP}, \mathrm{NDI}$, and $\mathrm{NDI}_{2}$ moieties 
were estimated as $B_{Z n T A P}=0.75 \mathrm{mT}, B_{N D I_{1}}=0.38 \mathrm{mT}$, and $B_{N D I_{2}}=0.27 \mathrm{mT}$. These values were then used to estimate the mean hyperfine fields as $1.46 \mathrm{mT}$ for NDI 1 and $1.38 \mathrm{mT}$ for $\mathrm{NDI}_{2}$. The rate constants of hyperfine mixing were estimated using a model described previously: ${ }^{13}$

$$
k_{h f i} \approx \frac{1}{2 \pi} g \mu_{B} B_{h f i}
$$

where $g$ is the mean $g$-value of the radical, $\mu_{B}$ is the Bohr magneton, and $B_{h f i}$ is the mean hyperfine field as described and estimated above. This gives estimated hyperfine mixing rate constants of $4.1 \times 10^{6} \mathrm{~s}^{-1}$ and $3.9 \times 10^{6} \mathrm{~s}^{-1}$ for ZnP-An-NDI and ZnP-An-NDI 2 , respectively.

\section{Results of DFT and TDDFT Computations}

\subsection{Internal Reorganization Energy Computations}

The internal reorganization energy $\lambda_{i}$ can be decomposed into contributions from the cation and anion moieties, $\lambda_{i}=\lambda_{i+}+\lambda_{i-}$, which were determined via density functional theory (DFT) computations. The calculations were performed on the molecular fragments in neutral, singly oxidized (for $\mathrm{ZnP}-\mathrm{Ph}$ ), and/or singly reduced (for An-NDI and An-NDI 2 ) states to calculate the internal reorganization energy $\left(\lambda_{i}\right)$ of each unit. This quantity can be calculated using the singlepoint energies of the ionic species at their respective optimized geometries and the energies of the ionic species at the optimized neutral geometries, as shown in eq S7.

$$
\lambda_{i}^{+/-}=E_{\text {neutral }}^{+/-}-E_{\text {opt }}^{+/-}
$$

where $\lambda_{i}^{+/-}$is the internal reorganization energy for oxidation of the donor and reduction of the acceptor, $E_{\text {neutral }}^{+/-}$is the energy of the oxidized donor or reduced acceptor at the neutral geometry, and $E_{\text {opt }}^{+/-}$is the energy of the oxidized donor or reduced acceptor at the optimized ionic geometry. The $\mathrm{ZnP}$ cation contribution is $\lambda_{i+}=0.05 \mathrm{eV}$ and the two NDI anion contributions are $\lambda_{i-}=0.19 \mathrm{eV}$ for $\mathbf{1}$ and $0.22 \mathrm{eV}$ for $\mathbf{2}$. When combined, $\lambda_{i}=0.24 \mathrm{eV}$ for $\mathbf{1}$ and 

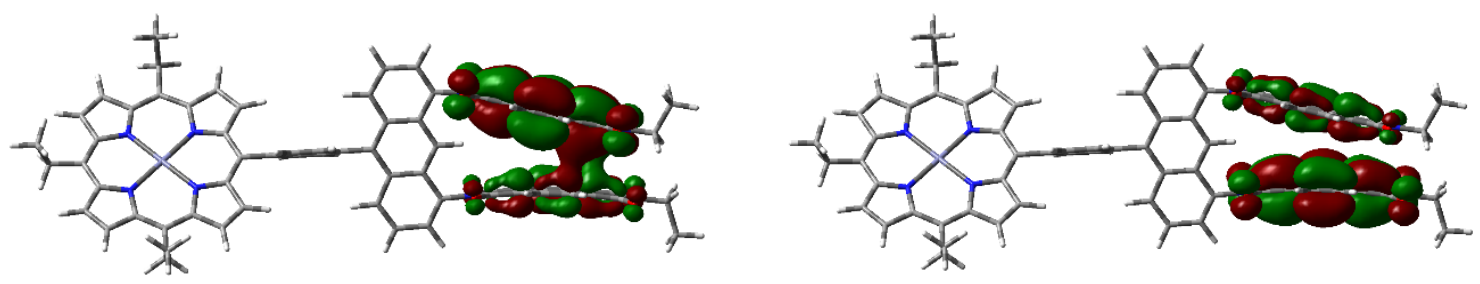

Figure S10. Singly occupied molecular orbitals of the lowest charge separated triplet ${ }^{3} \mathrm{D}^{+} \mathrm{A}_{2}{ }^{-}$ state computed using the pbe 0 functional. The wave functions are partially delocalized between the two NDI fragments.

\subsection{Computed Electronic Couplings}

Table S1. DA 2 singlet charge separation coupling $\left({ }^{1} \mathrm{D}^{*} \mathrm{~A}_{2} \rightarrow{ }^{1} \mathrm{D}^{+} \mathrm{A}_{2}{ }^{-}\right)$evaluated at the FranckCondon geometry.

\begin{tabular}{c|c|c} 
& LE1 $(\mathbf{m e V})$ & LE2 $(\mathbf{m e V})$ \\
\hline CS1 & 1.76 & 0.14 \\
\hline CS2 & 0.76 & 0.16 \\
\hline CS3 & 0.05 & 0.86 \\
\hline CS4 & 0.10 & 0.41
\end{tabular}

Table S2. DA 2 singlet charge separation coupling $\left({ }^{1} \mathrm{D}^{*} \mathrm{~A}_{2} \rightarrow{ }^{1} \mathrm{D}^{+} \mathrm{A}_{2}^{-}\right)$evaluated at the optimized minimum geometry of the initial locally excited state $\left(\mathrm{D}^{*} \mathrm{~A}_{2}, \mathrm{LE} 1\right)$.

\begin{tabular}{c|c|c} 
& LE1 $(\mathbf{m e V})$ & LE2 $(\mathbf{m e V})$ \\
\hline CS1 & 1.50 & 0.16 \\
\hline CS2 & 0.69 & 0.14 \\
\hline CS3 & 0.06 & 0.68 \\
\hline CS4 & 0.09 & 0.38
\end{tabular}

Table S3. DA charge separation coupling $\left({ }^{1} \mathrm{D}^{*} \mathrm{~A} \rightarrow{ }^{1} \mathrm{D}^{+} \mathrm{A}^{-}\right)$evaluated at the Franck-Condon geometry.

\begin{tabular}{c|c|c} 
& LE1 $(\mathbf{m e V})$ & LE2 $(\mathbf{m e V})$ \\
\hline CS1 & 2.12 & 0.15 \\
\hline CS2 & 0.06 & 1.21
\end{tabular}


Table S4. DA charge separation coupling $\left({ }^{1} \mathrm{D}^{*} \mathrm{~A} \rightarrow{ }^{1} \mathrm{D}^{+} \mathrm{A}^{-}\right)$evaluated at the optimized minimum geometry of the initial locally excited state ( $\left.{ }^{*} \mathrm{~A}, \mathrm{LE} 1\right)$.

\begin{tabular}{c|c|c} 
& LE1 $(\mathbf{m e V})$ & LE2 $(\mathbf{m e V})$ \\
\hline CS1 & 2.08 & 0.93 \\
\hline CS2 & 0.41 & 0.98
\end{tabular}

Table S5. $\mathrm{DA}_{2}$ charge recombination coupling $\left({ }^{3} \mathrm{D}^{+} \mathrm{A}_{2}{ }^{-}{ }^{3} \mathrm{D}^{*} \mathrm{~A}_{2}\right)$ evaluated at the optimized geometry of the lowest local triplet excited state $\left({ }^{3} \mathrm{D}^{*} \mathrm{~A}_{2}, \mathrm{LE} 1\right)$. LE and CS refer to the locally excited state and charge separated state respectively.

\begin{tabular}{c|c|c} 
& LE1 $(\mathbf{m e V})$ & LE2 $(\mathbf{m e V})$ \\
\hline CS1 & 0.62 & 0.76 \\
\hline CS2 & 0.28 & 0.49 \\
\hline CS3 & 0.14 & 0.29 \\
\hline CS4 & 0.12 & 0.15
\end{tabular}

Table S6. DA 2 charge recombination coupling $\left({ }^{3} \mathrm{D}^{+} \mathrm{A}_{2}-{ }^{3} \mathrm{D}^{*} \mathrm{~A}_{2}\right)$ evaluated at the optimized geometry of the lowest charge separated excited state $\left({ }^{3} \mathrm{D}^{+} \mathrm{A}_{2}{ }^{-}, \mathrm{CS} 1\right)$. LE and CS refer to the locally excited state and charge separated state respectively.

\begin{tabular}{c|c|c} 
& LE1 $(\mathbf{m e V})$ & LE2 $(\mathbf{m e V})$ \\
\hline CS1 & 2.65 & 1.22 \\
\hline CS2 & 0.10 & 0.11 \\
\hline CS3 & 0.11 & 0.27 \\
\hline CS4 & 0.10 & 0.15
\end{tabular}

Table S7. DA charge recombination coupling $\left({ }^{3} \mathrm{D}^{+} \mathrm{A}^{-} \rightarrow{ }^{3} \mathrm{D}^{*} \mathrm{~A}\right)$ evaluated at the optimized geometry of the lowest locally excited state $\left({ }^{3} \mathrm{D}^{*} \mathrm{~A}, \mathrm{LE} 1\right)$. LE and CS refer to the locally excited state and charge separated state respectively.

\begin{tabular}{c|c|c} 
& LE1 $(\mathbf{m e V})$ & LE2 $(\mathbf{m e V})$ \\
\hline CS1 & 0.80 & 0.95 \\
\hline CS2 & 0.19 & 0.40
\end{tabular}

Table S8. DA charge recombination coupling $\left({ }^{3} \mathrm{D}^{+} \mathrm{A}^{-} \rightarrow{ }^{3} \mathrm{D}^{*} \mathrm{~A}\right)$ evaluated at the optimized geometry of the lowest charge separated excited state $\left({ }^{3} \mathrm{D}^{+} \mathrm{A}^{-}, \mathrm{CS} 1\right)$. LE and CS refer to the locally excited state and charge separated state respectively.

\begin{tabular}{c|c|c} 
& LE1 $(\mathbf{m e V})$ & LE2 $(\mathbf{m e V})$ \\
\hline CS1 & 2.90 & 22.38 \\
\hline CS2 & 0.40 & 1.47
\end{tabular}




\subsection{Selected IR Peak Assignments and Intensities}

Table S9: 1 (Ground State)

\begin{tabular}{|c|c|c|c|c|}
\hline $\operatorname{Exp} .\left(\mathrm{cm}^{-1}\right)$ & Calc. $\left(\mathrm{cm}^{-1}\right)$ & Strength & Intensity $\left(\mathrm{km} \mathrm{mol}^{-1}\right)$ & Assignment \\
\hline 1345 & 1432 & strong & 272.9 & $\mathrm{ZnP} \omega\left(\mathrm{C}-\mathrm{H}_{2}\right), \mathrm{An} \rho(\mathrm{C}-\mathrm{H})$ \\
\hline 1369 & 1436.9 & weak & 128.7 & Cy. hex. dicarb. $\omega\left(\mathrm{C}-\mathrm{H}_{2}\right)$ \\
\hline 1455 & 1505 & weak & 319.3 & NDI $v(\mathrm{C}=\mathrm{C})$ \\
\hline 1584 & 1655 & weak & 196 & NDI $v(C=C)$ \\
\hline 1671 & 1772 & strong & 669.8 & NDI $v(\mathrm{C}=\mathrm{O})$ \\
\hline 1689 & 1791.5 & strong & 374.5 & $\mathrm{NDI} v(\mathrm{C}=\mathrm{O})$ \\
\hline 1709 & 1814.2 & strong & 532.2 & NDI $v(\mathrm{C}=\mathrm{O})$ \\
\hline 1722 & 1832.1 & strong & 218.3 & NDI $v(\mathrm{C}=\mathrm{O})$ \\
\hline
\end{tabular}

Table S10: 1 (Charge-Separated State)

\begin{tabular}{l|l|l|l|l} 
Exp. $\left(\mathbf{c m}^{-\mathbf{1}}\right)$ & Calc. $\left(\mathbf{c m}^{-\mathbf{1}}\right)$ & Strength & Intensity $\left(\mathbf{k m ~ m o l} \mathbf{~ m}^{\mathbf{1}}\right)$ & Assignment \\
\hline 1265 & 1347 & strong & 1305.7 & $\mathrm{ZnP} \omega\left(\mathrm{C}-\mathrm{H}_{2}\right)$ \\
\hline 1331 & 1419 & weak & 259.3 & $\mathrm{ZnP} \omega\left(\mathrm{C}-\mathrm{H}_{2}\right)$ \\
\hline 1410 & 1478.6 & weak & 233.3 & $\mathrm{ZnP} \omega\left(\mathrm{C}-\mathrm{H}_{2}\right)$ \\
\hline 1519 & 1583 & strong & 1076.9 & NDI $v(\mathrm{C}=\mathrm{C})$ \\
\hline 1602 & 1653.2 & strong & 280.3 & NDI $v(\mathrm{C}=\mathrm{C})$ \\
\hline 1637 & 1701 & strong & 459 & NDI $v(\mathrm{C}=\mathrm{O})$ \\
\hline 1698 & 1737 & strong & 2408.3 & NDI $v(\mathrm{C}=\mathrm{O})$
\end{tabular}

Table S11: 2 (Ground State)

\begin{tabular}{|c|c|c|c|c|}
\hline $\operatorname{Exp} .\left(\mathrm{cm}^{-1}\right)$ & Calc. $\left(\mathrm{cm}^{-1}\right)$ & Strength & Intensity $\left(\mathrm{km} \mathrm{mol}^{-1}\right)$ & Assignment \\
\hline 1345 & 1406 & strong & 157.6 & $\mathrm{ZnP} \omega\left(\mathrm{C}-\mathrm{H}_{2}\right), \mathrm{An} \rho(\mathrm{C}-\mathrm{H})$ \\
\hline 1369 & 1423 & weak & 173.2 & Cy. hex. dicarb. $\omega\left(\mathrm{C}-\mathrm{H}_{2}\right)$ \\
\hline 1455 & 1507 & weak & 561.3 & $\mathrm{NDI} v(\mathrm{C}=\mathrm{C})$ \\
\hline 1584 & 1657.2 & weak & 404.8 & NDI $v(\mathrm{C}=\mathrm{C})$ \\
\hline 1671 & 1778.3 & strong & 1210 & NDI $v(\mathrm{C}=\mathrm{O})$ \\
\hline 1689 & 1792.1 & strong & 798.2 & NDI $v(\mathrm{C}=\mathrm{O})$ \\
\hline 1709 & 1817.2 & strong & 1017.4 & NDI $v(\mathrm{C}=\mathrm{O})$ \\
\hline 1722 & 1831.2 & strong & 249.2 & NDI $v(C=O)$ \\
\hline
\end{tabular}

Table S12: 2 (Charge-Separated State)

\begin{tabular}{|c|c|c|c|c|}
\hline $\operatorname{Exp} .\left(\mathrm{cm}^{-1}\right)$ & Calc. $\left(\mathrm{cm}^{-1}\right)$ & Strength & Intensity $\left(\mathrm{km} \mathrm{mol}^{-1}\right)$ & Assignment \\
\hline 1265 & 1321 & strong & 3634 & $\mathrm{ZnP} \omega\left(\mathrm{C}-\mathrm{H}_{2}\right)$ \\
\hline 1331 & 1389 & weak & 788.4 & $\mathrm{ZnP} \omega\left(\mathrm{C}-\mathrm{H}_{2}\right)$ \\
\hline 1410 & 1481 & weak & 1001.7 & $\mathrm{ZnP} \omega\left(\mathrm{C}-\mathrm{H}_{2}\right)$ \\
\hline 1519 & 1596.5 & strong & 892 & NDI $v(C=C)$ \\
\hline 1602 & 1652.4 & strong & 1479.7 & NDI $v(\mathrm{C}=\mathrm{C})$ \\
\hline 1637 & 1743 & strong & 301.7 & NDI $v(\mathrm{C}=\mathrm{O})$ \\
\hline 1698 & 1775 & strong & 747.4 & NDI $v(\mathrm{C}=\mathrm{O})$ \\
\hline
\end{tabular}




\subsection{Cartesian Coordinates}

Optimized single NDI acceptor (DA) structure on the ground state.

\begin{tabular}{|c|c|c|c|}
\hline C & -4.82275098 & 2.46200411 & -0.43248448 \\
\hline $\mathrm{C}$ & -5.76573052 & 3.43515565 & -0.57272945 \\
\hline C & -7.05576538 & 2.78476313 & -0.53255464 \\
\hline $\mathrm{N}$ & -6.87715969 & 1.43565202 & -0.37734446 \\
\hline $\mathrm{C}$ & -5.53113576 & 1.20928634 & -0.32227114 \\
\hline $\mathrm{C}$ & -5.88651565 & -3.48730143 & 0.18417435 \\
\hline C & -4.90950354 & -2.54374645 & 0.07851540 \\
\hline C & -5.57421360 & -1.26687977 & -0.02596118 \\
\hline $\mathrm{N}$ & -6.92744053 & -1.44832608 & 0.01230864 \\
\hline C & -7.15335317 & -2.79283610 & 0.14357768 \\
\hline C & -4.90552842 & -0.03926406 & -0.16806179 \\
\hline $\mathrm{C}$ & -11.86848376 & -2.39966792 & 0.28217358 \\
\hline $\mathrm{C}$ & -10.92329556 & -3.37692597 & 0.36566721 \\
\hline $\mathrm{C}$ & -9.63856454 & -2.73643644 & 0.21339696 \\
\hline $\mathrm{N}$ & -9.82232203 & -1.39080127 & 0.04539244 \\
\hline $\mathrm{C}$ & -11.17017093 & -1.15324247 & 0.07471022 \\
\hline C & -8.40752966 & -3.41985613 & 0.23799446 \\
\hline $\mathrm{C}$ & -10.80408865 & 3.52738914 & -0.54972685 \\
\hline $\mathrm{C}$ & -11.78288346 & 2.59324151 & -0.39263497 \\
\hline $\mathrm{C}$ & -11.12789596 & 1.31375404 & -0.25747134 \\
\hline $\mathrm{N}$ & -9.77245839 & 1.49105616 & -0.32981089 \\
\hline $\mathrm{C}$ & -9.54194116 & 2.82787921 & -0.50872576 \\
\hline $\mathrm{C}$ & -8.28689032 & 3.45637398 & -0.62183554 \\
\hline $\mathrm{C}$ & -11.80476505 & 0.09357127 & -0.07623552 \\
\hline $\mathrm{Zn}$ & -8.34938673 & 0.02116298 & -0.16818537 \\
\hline $\mathrm{H}$ & -3.74243661 & 2.58210878 & -0.40100115 \\
\hline $\mathrm{H}$ & -5.58617761 & 4.50245213 & -0.67648826 \\
\hline $\mathrm{H}$ & -5.74362668 & -4.56082477 & 0.28006599 \\
\hline $\mathrm{H}$ & -3.83338392 & -2.69935434 & 0.06181260 \\
\hline $\mathrm{H}$ & -12.94481488 & -2.53158741 & 0.35992519 \\
\hline $\mathrm{H}$ & -11.10036980 & -4.43793018 & 0.52346918 \\
\hline $\mathrm{H}$ & -10.94423807 & 4.59811047 & -0.67582750 \\
\hline $\mathrm{H}$ & -12.85435272 & 2.77566855 & -0.36906773 \\
\hline $\mathrm{C}$ & -13.31270070 & 0.13460120 & 0.03470092 \\
\hline $\mathrm{H}$ & -13.73930239 & -0.79302213 & -0.36980056 \\
\hline $\mathrm{H}$ & -13.70980293 & 0.93187866 & -0.60787641 \\
\hline $\mathrm{C}$ & -13.80260342 & 0.34081511 & 1.46686552 \\
\hline $\mathrm{H}$ & -14.90280764 & 0.36485099 & 1.50772572 \\
\hline $\mathrm{H}$ & -13.45475014 & -0.46979651 & 2.12516383 \\
\hline $\mathrm{H}$ & -13.42532423 & 1.28777794 & 1.88203217 \\
\hline $\mathrm{C}$ & -8.26170693 & 4.96169393 & -0.76651237 \\
\hline $\mathrm{H}$ & -7.38419929 & 5.26097260 & -1.35555279 \\
\hline $\mathrm{H}$ & -9.12602769 & 5.29011105 & -1.35975556 \\
\hline $\mathrm{C}$ & -8.25224233 & 5.69537862 & 0.57344560 \\
\hline $\mathrm{H}$ & -7.37042824 & 5.41762894 & 1.17067432 \\
\hline $\mathrm{H}$ & -8.23387893 & 6.78645136 & 0.42552024 \\
\hline $\mathrm{H}$ & -9.14443454 & 5.44700272 & 1.16813295 \\
\hline $\mathrm{C}$ & -8.43385496 & -4.91629963 & 0.45504813 \\
\hline $\mathrm{H}$ & -9.33203703 & -5.34255715 & -0.01177055 \\
\hline $\mathrm{H}$ & -7.59166254 & -5.38081196 & -0.07558655 \\
\hline $\mathrm{C}$ & 3870 & -5.3102 & 1. \\
\hline
\end{tabular}




\begin{tabular}{|c|c|c|c|}
\hline $\mathrm{H}$ & -9.24452185 & -4.89278779 & 2.47969309 \\
\hline $\mathrm{H}$ & -8.40765592 & -6.40510639 & 2.04697253 \\
\hline $\mathrm{H}$ & -7.47176659 & -4.93352000 & 2.41170108 \\
\hline $\mathrm{C}$ & -3.41639304 & -0.06446761 & -0.15773755 \\
\hline $\mathrm{C}$ & -2.68771870 & 0.21520247 & -1.32063273 \\
\hline C & -2.71287884 & -0.37007819 & 1.01406345 \\
\hline C & -1.29514102 & 0.18905885 & -1.31322801 \\
\hline $\mathrm{H}$ & -3.22334185 & 0.44919607 & -2.24370994 \\
\hline C & -1.32023271 & -0.39520146 & 1.02368360 \\
\hline $\mathrm{H}$ & -3.26801963 & -0.58498204 & 1.93022698 \\
\hline C & -0.59535318 & -0.11645018 & -0.14049855 \\
\hline $\mathrm{H}$ & -0.73941779 & 0.40435972 & -2.22905574 \\
\hline $\mathrm{H}$ & -0.78386839 & -0.63169116 & 1.94581130 \\
\hline $\mathrm{C}$ & 0.89155110 & -0.14683720 & -0.13177466 \\
\hline $\mathrm{C}$ & 1.61379900 & 1.00706351 & 0.23932998 \\
\hline C & 1.56820110 & -1.33051455 & -0.49292188 \\
\hline C & 3.05095218 & 0.96921264 & 0.24204667 \\
\hline C & 0.96738524 & 2.22760258 & 0.60517617 \\
\hline $\mathrm{C}$ & 3.00463860 & -1.35070636 & -0.48157455 \\
\hline C & 0.87867809 & -2.52482707 & -0.86840605 \\
\hline $\mathrm{C}$ & 3.77054737 & 2.14871338 & 0.62130220 \\
\hline $\mathrm{C}$ & 3.70832929 & -0.20288485 & -0.12267758 \\
\hline C & 1.69435048 & 3.33430221 & 0.95564153 \\
\hline $\mathrm{H}$ & -0.12304399 & 2.26540804 & 0.60033858 \\
\hline C & 3.67548097 & -2.56571389 & -0.83449984 \\
\hline $\mathrm{C}$ & 1.56437384 & -3.66105130 & -1.20762117 \\
\hline $\mathrm{H}$ & -0.21233161 & -2.51825478 & -0.88009806 \\
\hline $\mathrm{C}$ & 3.11466929 & 3.29756538 & 0.96733084 \\
\hline $\mathrm{H}$ & 4.79590769 & -0.21669098 & -0.11984575 \\
\hline $\mathrm{H}$ & 1.18366361 & 4.25918132 & 1.23091650 \\
\hline $\mathrm{C}$ & 2.98570482 & -3.68766600 & -1.18808607 \\
\hline $\mathrm{H}$ & 1.01924363 & -4.56325084 & -1.49182174 \\
\hline $\mathrm{H}$ & 3.68836761 & 4.18248218 & 1.24776763 \\
\hline $\mathrm{H}$ & 3.52790477 & -4.59800319 & -1.44834483 \\
\hline $\mathrm{C}$ & 5.96373727 & 1.46022826 & 1.57227621 \\
\hline C & 5.96199527 & 2.57911107 & -0.46642217 \\
\hline $\mathrm{N}$ & 5.19113159 & 2.09957339 & 0.59935928 \\
\hline O & 5.52017010 & 0.90702935 & 2.54674352 \\
\hline 0 & 5.54038599 & 3.25108748 & -1.37180097 \\
\hline C & 5.68438343 & -2.76390867 & 0.48411030 \\
\hline C & 5.77857598 & -2.05825031 & -1.90799988 \\
\hline C & 7.08992234 & -2.32225725 & 0.65508451 \\
\hline C & 7.19355419 & -1.65413599 & -1.71562054 \\
\hline $\mathrm{C}$ & 7.79052042 & -1.76354310 & -0.43841633 \\
\hline $\mathrm{C}$ & 7.68540169 & -2.39411510 & 1.90198581 \\
\hline $\mathrm{C}$ & 7.89555713 & -1.08935885 & -2.76687447 \\
\hline $\mathrm{C}$ & 9.10211819 & -1.26275098 & -0.23978575 \\
\hline $\mathrm{C}$ & 8.98699462 & -1.89830694 & 2.09763120 \\
\hline $\mathrm{H}$ & 7.11684143 & -2.81718539 & 2.73158508 \\
\hline $\mathrm{C}$ & 9.19939854 & -0.60080880 & -2.57033248 \\
\hline $\mathrm{H}$ & 7.40828421 & -1.00818425 & -3.73994882 \\
\hline $\mathrm{C}$ & 9.68513531 & -1.33077610 & 1.04595580 \\
\hline C & 9.79255866 & -0.67297977 & -1.3217277 \\
\hline $\mathrm{H}$ & 9.45580862 & -1.93282212 & 3.082419 \\
\hline
\end{tabular}




$\begin{array}{rrrr}\mathrm{H} & 9.75219784 & -0.13721370 & -3.38901054 \\ \mathrm{C} & 11.02932569 & -0.74810868 & 1.27752869 \\ \mathrm{C} & 11.14034068 & -0.09115959 & -1.10712757 \\ \mathrm{~N} & 11.64627302 & -0.13105548 & 0.19019620 \\ \mathrm{O} & 11.56979902 & -0.78214541 & 2.36444468 \\ \mathrm{O} & 11.77228939 & 0.42109356 & -2.00919972 \\ \mathrm{C} & 12.96276383 & 0.46805980 & 0.41669620 \\ \mathrm{H} & 13.04797026 & 1.31502357 & -0.27482407 \\ \mathrm{H} & 12.96497484 & 0.84641466 & 1.44614034 \\ \mathrm{C} & 14.09486527 & -0.51998207 & 0.20229472 \\ \mathrm{H} & 14.09701457 & -0.89958889 & -0.82959580 \\ \mathrm{H} & 15.06031217 & -0.02560659 & 0.38442848 \\ \mathrm{H} & 14.01275569 & -1.37012831 & 0.89465911 \\ \mathrm{~N} & 5.10830449 & -2.53606627 & -0.77543590 \\ \mathrm{O} & 5.03770125 & -3.24884465 & 1.38356928 \\ \mathrm{O} & 5.20467111 & -1.93728972 & -2.96699346 \\ \mathrm{C} & 8.49614711 & 2.85599394 & -0.85857711 \\ \mathrm{H} & 9.33403335 & 2.18278986 & -1.09953476 \\ \mathrm{H} & 8.16604359 & 3.29196210 & -1.81391023 \\ \mathrm{C} & 8.99201683 & 3.93183005 & 0.09832670 \\ \mathrm{H} & 8.23425967 & 4.72933429 & 0.19783330 \\ \mathrm{H} & 9.89072184 & 4.40893778 & -0.32225438 \\ \mathrm{C} & 9.28315089 & 3.34501932 & 1.47373927 \\ \mathrm{H} & 9.69267625 & 4.11465079 & 2.14630840 \\ \mathrm{H} & 10.05629595 & 2.55881063 & 1.39016036 \\ \mathrm{C} & 8.01415633 & 2.76320660 & 2.07894275 \\ \mathrm{H} & 7.27606620 & 3.57703116 & 2.19300273 \\ \mathrm{H} & 8.18886299 & 2.35901749 & 3.08693036 \\ \mathrm{C} & 7.41716147 & 1.65268864 & 1.19768093 \\ \mathrm{H} & 7.96129089 & 0.71599192 & 1.37866466 \\ \mathrm{C} & 7.35966076 & 2.01650899 & -0.29090482 \\ \mathrm{H} & 7.30816975 & 1.07662007 & -0.87096856\end{array}$

Optimized single NDI acceptor (DA) structure of the triplet local excited state.

$\begin{array}{lrrr}\mathrm{C} & -4.87738470 & 2.50846907 & -0.69955081 \\ \mathrm{C} & -5.84817813 & 3.43920239 & -0.89869064 \\ \mathrm{C} & -7.12015667 & 2.74994606 & -0.81801743 \\ \mathrm{~N} & -6.90139977 & 1.41738044 & -0.58768956 \\ \mathrm{C} & -5.54740701 & 1.23810652 & -0.52086189 \\ \mathrm{C} & -5.83315161 & -3.45958615 & 0.19662326 \\ \mathrm{C} & -4.86195598 & -2.48588975 & -0.01550004 \\ \mathrm{C} & -5.54313973 & -1.24897959 & -0.08313846 \\ \mathrm{~N} & -6.89130328 & -1.44153724 & 0.07184805 \\ \mathrm{C} & -7.07649743 & -2.78800418 & 0.25035750 \\ \mathrm{C} & -4.89835375 & 0.01699270 & -0.30091136 \\ \mathrm{C} & -11.80272496 & -2.47053456 & 0.78955806 \\ \mathrm{C} & -10.83710666 & -3.42186328 & 0.87910184 \\ \mathrm{C} & -9.58177043 & -2.78515037 & 0.53742716 \\ \mathrm{~N} & -9.80650518 & -1.46335735 & 0.25346775 \\ \mathrm{C} & -11.15082802 & -1.24143051 & 0.38456875 \\ \mathrm{C} & -8.34307709 & -3.43460216 & 0.50046224 \\ \mathrm{C} & -10.89227207 & 3.36062735 & -0.81778735\end{array}$




\begin{tabular}{|c|c|c|c|}
\hline C & -11.85788312 & 2.41030147 & -0.50420077 \\
\hline C & -11.16877766 & 1.20601362 & -0.23199652 \\
\hline $\mathrm{N}$ & -9.81827387 & 1.39966276 & -0.36815743 \\
\hline $\mathrm{C}$ & -9.63922786 & 2.71248376 & -0.72468257 \\
\hline $\mathrm{C}$ & -8.37160080 & 3.36582354 & -0.93458299 \\
\hline $\mathrm{C}$ & -11.81069940 & -0.02917996 & 0.14602476 \\
\hline $\mathrm{Zn}$ & -8.35407577 & -0.02135571 & -0.16006750 \\
\hline $\mathrm{H}$ & -3.80211728 & 2.66629170 & -0.66739013 \\
\hline $\mathrm{H}$ & -5.70323278 & 4.50461104 & -1.06022571 \\
\hline $\mathrm{H}$ & -5.66286957 & -4.52786151 & 0.30325640 \\
\hline $\mathrm{H}$ & -3.79039621 & -2.63437450 & -0.12279451 \\
\hline $\mathrm{H}$ & -12.86346499 & -2.60278874 & 0.98731741 \\
\hline $\mathrm{H}$ & -10.97497517 & -4.46218422 & 1.16326846 \\
\hline $\mathrm{H}$ & -11.07215540 & 4.40089236 & -1.07675602 \\
\hline $\mathrm{H}$ & -12.93261073 & 2.56996708 & -0.47471045 \\
\hline $\mathrm{C}$ & -13.30025932 & 0.02299871 & 0.36377747 \\
\hline $\mathrm{H}$ & -13.74509683 & -0.95997944 & 0.16138041 \\
\hline $\mathrm{H}$ & -13.75200725 & 0.70476819 & -0.36986441 \\
\hline C & -13.67370405 & 0.47157547 & 1.77753110 \\
\hline $\mathrm{H}$ & -14.76719311 & 0.52006651 & 1.89469512 \\
\hline $\mathrm{H}$ & -13.28064013 & -0.22865178 & 2.53020076 \\
\hline $\mathrm{H}$ & -13.26153572 & 1.46692014 & 1.99989546 \\
\hline $\mathrm{C}$ & -8.40897283 & 4.84707517 & -1.20644919 \\
\hline $\mathrm{H}$ & -7.53241284 & 5.14009697 & -1.79943191 \\
\hline $\mathrm{H}$ & -9.27934161 & 5.07819727 & -1.83628511 \\
\hline C & -8.46506816 & 5.68492092 & 0.07208232 \\
\hline $\mathrm{H}$ & -7.57393177 & 5.51459802 & 0.69503486 \\
\hline $\mathrm{H}$ & -8.51355757 & 6.75817826 & -0.16775193 \\
\hline $\mathrm{H}$ & -9.34788007 & 5.42904281 & 0.67619022 \\
\hline $\mathrm{C}$ & -8.29825281 & -4.90495254 & 0.82294045 \\
\hline $\mathrm{H}$ & -9.24483111 & -5.38143612 & 0.53615454 \\
\hline $\mathrm{H}$ & -7.52841364 & -5.38749690 & 0.20455320 \\
\hline $\mathrm{C}$ & -8.01367420 & -5.17954332 & 2.30045624 \\
\hline $\mathrm{H}$ & -8.80287391 & -4.75534953 & 2.93959356 \\
\hline $\mathrm{H}$ & -7.96411802 & -6.26230447 & 2.49241715 \\
\hline $\mathrm{H}$ & -7.05718034 & -4.73342347 & 2.61023075 \\
\hline $\mathrm{C}$ & -3.41473619 & 0.01188393 & -0.29250777 \\
\hline $\mathrm{C}$ & -2.68310310 & 0.33980602 & -1.44134936 \\
\hline $\mathrm{C}$ & -2.71452061 & -0.33654722 & 0.87100297 \\
\hline $\mathrm{C}$ & -1.29036489 & 0.31634195 & -1.42893763 \\
\hline $\mathrm{H}$ & -3.21472468 & 0.60133484 & -2.35920178 \\
\hline $\mathrm{C}$ & -1.32345413 & -0.34855430 & 0.88666615 \\
\hline $\mathrm{H}$ & -3.27229299 & -0.59705814 & 1.77316873 \\
\hline C & -0.59394657 & -0.02766172 & -0.26504927 \\
\hline $\mathrm{H}$ & -0.73249674 & 0.56337350 & -2.33539013 \\
\hline $\mathrm{H}$ & -0.78997218 & -0.61633093 & 1.80185783 \\
\hline $\mathrm{C}$ & 0.89188923 & -0.06807702 & -0.24895247 \\
\hline $\mathrm{C}$ & 1.61794930 & 0.99746063 & 0.32576183 \\
\hline $\mathrm{C}$ & 1.56496777 & -1.17986860 & -0.79892138 \\
\hline $\mathrm{C}$ & 3.05453289 & 0.94073318 & 0.34914123 \\
\hline $\mathrm{C}$ & 0.97750923 & 2.15094401 & 0.87426671 \\
\hline $\mathrm{C}$ & 3.00066766 & -1.21646845 & -0.7692399 \\
\hline $\mathrm{C}$ & 0.87444277 & -2.29062675 & -1.3759386 \\
\hline $\mathrm{C}$ & 3.77855722 & 2.02770601 & $0.938144 \varepsilon$ \\
\hline
\end{tabular}




\begin{tabular}{|c|c|c|c|}
\hline C & 3.70751564 & -0.15877551 & -0.20119503 \\
\hline$C$ & 1.70849777 & 3.17217129 & 1.42102223 \\
\hline $\mathrm{H}$ & -0.11163876 & 2.20908068 & 0.84870517 \\
\hline C & 3.66970199 & -2.35694221 & -1.31917695 \\
\hline $\mathrm{C}$ & 1.55847213 & -3.35697795 & -1.89692618 \\
\hline $\mathrm{H}$ & -0.21627583 & -2.27916877 & -1.39429850 \\
\hline C & 3.12723087 & 3.11130705 & 1.45950288 \\
\hline $\mathrm{H}$ & 4.79430404 & -0.18888965 & -0.17832351 \\
\hline $\mathrm{H}$ & 1.20192030 & 4.04713994 & 1.83303215 \\
\hline C & 2.97928888 & -3.39575416 & -1.86993810 \\
\hline $\mathrm{H}$ & 1.01141674 & -4.19507955 & -2.33298706 \\
\hline $\mathrm{H}$ & 3.70377455 & 3.92755577 & 1.89793571 \\
\hline $\mathrm{H}$ & 3.51995261 & -4.25113966 & -2.27778360 \\
\hline $\mathrm{C}$ & 5.93908517 & 1.16287342 & 1.81669964 \\
\hline $\mathrm{C}$ & 6.00600736 & 2.62261372 & 0.00778013 \\
\hline $\mathrm{N}$ & 5.19904440 & 1.96392068 & 0.94305758 \\
\hline O & 5.46265070 & 0.44621452 & 2.66031973 \\
\hline O & 5.61399217 & 3.44094640 & -0.78322220 \\
\hline C & 5.66197854 & -2.80091621 & -0.03483435 \\
\hline C & 5.78358062 & -1.66257233 & -2.25282915 \\
\hline $\mathrm{C}$ & 7.06536296 & -2.40107922 & 0.23106381 \\
\hline $\mathrm{C}$ & 7.19306793 & -1.29620093 & -1.96923151 \\
\hline $\mathrm{C}$ & 7.77618088 & -1.64482938 & -0.72902832 \\
\hline C & 7.64879987 & -2.71010075 & 1.44698829 \\
\hline C & 7.90537114 & -0.54299532 & -2.88696242 \\
\hline $\mathrm{C}$ & 9.08629325 & -1.19458870 & -0.42665577 \\
\hline $\mathrm{C}$ & 8.94924201 & -2.26498930 & 1.74495728 \\
\hline $\mathrm{H}$ & 7.07210282 & -3.28239483 & 2.17514255 \\
\hline $\mathrm{C}$ & 9.20703134 & -0.10266707 & -2.58824254 \\
\hline $\mathrm{H}$ & 7.42872606 & -0.27887264 & -3.83244487 \\
\hline $\mathrm{C}$ & 9.65808811 & -1.51002144 & 0.82680637 \\
\hline $\mathrm{C}$ & 9.78835636 & -0.41298537 & -1.37095660 \\
\hline $\mathrm{H}$ & 9.40938740 & -2.48950074 & 2.70860717 \\
\hline $\mathrm{H}$ & 9.76819889 & 0.50627500 & -3.29883890 \\
\hline $\mathrm{C}$ & 11.00392005 & -0.99240087 & 1.17451080 \\
\hline C & 11.13690775 & 0.10892852 & -1.03923119 \\
\hline $\mathrm{N}$ & 11.63317107 & -0.18364710 & 0.22925830 \\
\hline 0 & 11.53570578 & -1.23846203 & 2.23814833 \\
\hline 0 & 11.77844478 & 0.78095709 & -1.82167689 \\
\hline $\mathrm{C}$ & 12.95293336 & 0.34864342 & 0.57302889 \\
\hline $\mathrm{H}$ & 13.04826210 & 1.31339147 & 0.06023654 \\
\hline $\mathrm{H}$ & 12.95289722 & 0.51887442 & 1.65648418 \\
\hline $\mathrm{C}$ & 14.07852951 & -0.58825664 & 0.17412060 \\
\hline $\mathrm{H}$ & 14.08242082 & -0.76044983 & -0.91177894 \\
\hline $\mathrm{H}$ & 15.04677994 & -0.14650753 & 0.45155224 \\
\hline $\mathrm{H}$ & 13.98726205 & -1.55589831 & 0.68805924 \\
\hline $\mathrm{N}$ & 5.10158784 & -2.34565731 & -1.23848698 \\
\hline 0 & 5.00475151 & -3.44131874 & 0.75281551 \\
\hline 0 & 5.22208228 & -1.34879703 & -3.27833496 \\
\hline $\mathrm{C}$ & 8.54907606 & 2.98029021 & -0.21968432 \\
\hline $\mathrm{H}$ & 9.40730678 & 2.36722285 & -0.53822258 \\
\hline $\mathrm{H}$ & 8.25317263 & 3.57549161 & -1.09713868 \\
\hline $\mathrm{C}$ & 8.98674046 & 3.87356728 & 0.93317326 \\
\hline $\mathrm{H}$ & 8.21179710 & 4.63304754 & 1.13998024 \\
\hline
\end{tabular}




$\begin{array}{rrrr}\mathrm{H} & 9.89329110 & 4.42688465 & 0.64313734 \\ \mathrm{C} & 9.23226870 & 3.05376573 & 2.19343807 \\ \mathrm{H} & 9.60073265 & 3.69498214 & 3.00920788 \\ \mathrm{H} & 10.02172697 & 2.30310753 & 2.00457145 \\ \mathrm{C} & 7.95039630 & 2.36084356 & 2.63090193 \\ \mathrm{H} & 7.19628247 & 3.13476220 & 2.86001742 \\ \mathrm{H} & 8.09214838 & 1.78295847 & 3.55602385 \\ \mathrm{C} & 7.40429098 & 1.42193218 & 1.54091414 \\ \mathrm{H} & 7.95117191 & 0.46980172 & 1.57578283 \\ \mathrm{C} & 7.40259555 & 2.04447702 & 0.13955431 \\ \mathrm{H} & 7.39174250 & 1.22370946 & -0.60071889\end{array}$

\section{Optimized single NDI acceptor (DA) structure of the triplet charge separated excited state.}

$\begin{array}{ll}\mathrm{C} & -4.87171529 \\ \mathrm{C} & -5.84022978 \\ \mathrm{C} & -7.10536717 \\ \mathrm{~N} & -6.89876542 \\ \mathrm{C} & -5.54916573 \\ \mathrm{C} & -5.81163887 \\ \mathrm{C} & -4.85418080 \\ \mathrm{C} & -5.54244867 \\ \mathrm{~N} & -6.88481699 \\ \mathrm{C} & -7.07979881 \\ \mathrm{C} & -4.89564868 \\ \mathrm{C} & -11.79333201 \\ \mathrm{C} & -10.82677871 \\ \mathrm{C} & -9.57381520 \\ \mathrm{~N} & -9.78580017 \\ \mathrm{C} & -11.12979978 \\ \mathrm{C} & -8.32323554 \\ \mathrm{C} & -10.88093529 \\ \mathrm{C} & -11.83528529 \\ \mathrm{C} & -11.14647765 \\ \mathrm{~N} & -9.80209512 \\ \mathrm{C} & -9.60973549 \\ \mathrm{C} & -8.36412252 \\ \mathrm{C} & -11.79054955 \\ \mathrm{Zn} & -8.34094461 \\ \mathrm{H} & -3.79709252 \\ \mathrm{H} & -5.68798835 \\ \mathrm{H} & -5.64835403 \\ \mathrm{H} & -3.78140400 \\ \mathrm{H} & -12.85830223 \\ \mathrm{H} & -10.97383617 \\ \mathrm{H} & -11.04825325 \\ \mathrm{H} & -12.90853931 \\ \mathrm{C} & -13.28406745 \\ \mathrm{H} & -13.71443609 \\ \mathrm{H} & -13.72720964 \\ \mathrm{C} & -13.68376821 \\ \mathrm{H} & -14.77916315 \\ \mathrm{H} & -13.28950951 \\ & \\ \mathrm{H} & \end{array}$

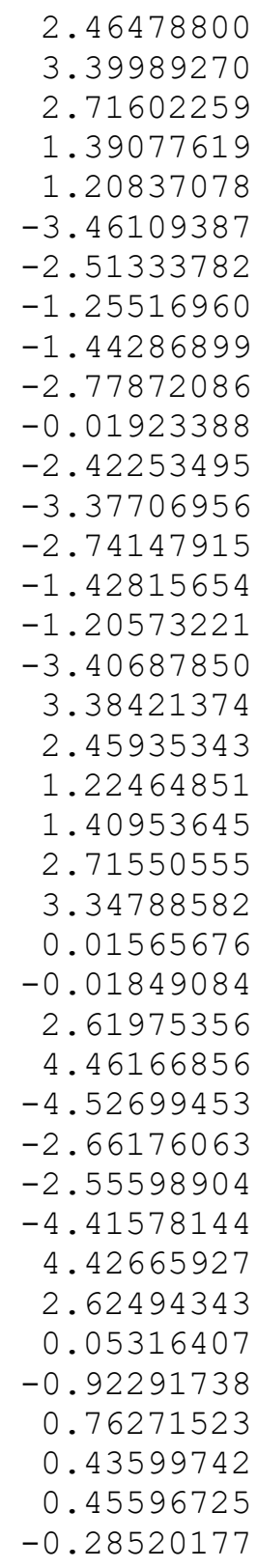

-0.70619529
-0.92623937
-0.84435603
-0.59783305
-0.52051253
0.29989431
0.08612815
-0.03387733
0.09262308
0.30761372
-0.27611615
0.73528343
0.85582228
0.54234996
0.24650094
0.34475644
0.53514908
-0.84757231
-0.54139931
-0.27102887
-0.40698718
-0.75857007
-0.97481771
0.08742945
-0.17354466
-0.66272022
-1.10061507
0.43229152
-0.00211870
0.90450618
1.13912447
-1.10449229
-0.50772800
0.27188960
0.01641409
-0.43809927
1.69887607
1.79454058
2.42985374
- 


\begin{tabular}{|c|c|c|c|}
\hline $\mathrm{H}$ & -13.29876747 & 1.43067045 & 1.96766122 \\
\hline $\mathrm{C}$ & -8.37299799 & 4.82184483 & -1.28006373 \\
\hline $\mathrm{H}$ & -7.50704112 & 5.07317678 & -1.90536128 \\
\hline $\mathrm{H}$ & -9.25404667 & 5.06674458 & -1.88704963 \\
\hline C & -8.36393826 & 5.68359533 & -0.01504380 \\
\hline $\mathrm{H}$ & -7.46998114 & 5.48773751 & 0.59501187 \\
\hline $\mathrm{H}$ & -8.36780051 & 6.75026304 & -0.28237094 \\
\hline $\mathrm{H}$ & -9.24634503 & 5.48400232 & 0.61043344 \\
\hline $\mathrm{C}$ & -8.31262939 & -4.87323299 & 0.87304713 \\
\hline $\mathrm{H}$ & -9.23933359 & -5.34246751 & 0.51979869 \\
\hline $\mathrm{H}$ & -7.50332725 & -5.37273153 & 0.32569602 \\
\hline C & -8.14542272 & -5.12218003 & 2.37423581 \\
\hline $\mathrm{H}$ & -8.96319959 & -4.66312249 & 2.94912384 \\
\hline $\mathrm{H}$ & -8.14500626 & -6.20196011 & 2.58222513 \\
\hline $\mathrm{H}$ & -7.19836388 & -4.70255329 & 2.74388870 \\
\hline $\mathrm{C}$ & -3.41561232 & -0.02109193 & -0.26513061 \\
\hline $\mathrm{C}$ & -2.68635489 & 0.32985532 & -1.40992429 \\
\hline $\mathrm{C}$ & -2.72120699 & -0.38494480 & 0.89773508 \\
\hline $\mathrm{C}$ & -1.29543857 & 0.30233588 & -1.39347970 \\
\hline $\mathrm{H}$ & -3.21453046 & 0.60177905 & -2.3267009 \\
\hline C & -1.33086666 & -0.38746862 & 0.9156719 \\
\hline $\mathrm{H}$ & -3.27756891 & -0.65171500 & 1.7992145. \\
\hline C & -0.59972165 & -0.05207259 & -0.2308429 \\
\hline $\mathrm{H}$ & -0.73420978 & 0.55780852 & -2.2949612 \\
\hline $\mathrm{H}$ & -0.79707791 & -0.66053799 & 1.8286021 \\
\hline C & 0.88555714 & -0.08323446 & -0.2114542 \\
\hline C & 1.59704527 & 0.96263756 & 0.4140792 \\
\hline C & 1.56541887 & -1.16495435 & -0.8101646 \\
\hline C & 3.03363433 & 0.91514208 & 0.4376350 \\
\hline C & 0.95062349 & 2.08937503 & 1.0100772 \\
\hline $\mathrm{C}$ & 3.00112036 & -1.18808156 & -0.7821674 \\
\hline $\mathrm{C}$ & 0.88920181 & -2.25899347 & -1.4342939 \\
\hline C & 3.75204345 & 1.97633470 & 1.0804727 \\
\hline C & 3.69644205 & -0.14992322 & -0.1660935 \\
\hline C & 1.67609858 & 3.08867090 & $1.6034524^{\circ}$ \\
\hline $\mathrm{H}$ & -0.13850309 & 2.14997190 & 0.98440031 \\
\hline C & 3.69560955 & -2.28987334 & -1.38039766 \\
\hline $\mathrm{C}$ & 1.59244773 & -3.29038354 & -1.9982654 \\
\hline $\mathrm{H}$ & -0.20151302 & -2.26758126 & -1.45529388 \\
\hline $\mathrm{C}$ & 3.09510012 & 3.03282409 & 1.6477236 \\
\hline $\mathrm{H}$ & 4.78388517 & -0.17693217 & -0.15376402 \\
\hline $\mathrm{H}$ & 1.16352770 & 3.94277172 & 2.05105900 \\
\hline $\mathrm{C}$ & 3.01387460 & -3.31085682 & -1.97395430 \\
\hline $\mathrm{H}$ & 1.05792068 & -4.11751532 & -2.47060903 \\
\hline $\mathrm{H}$ & 3.66697740 & 3.82871723 & 2.12718725 \\
\hline $\mathrm{H}$ & 3.56616104 & -4.13886190 & -2.42012702 \\
\hline $\mathrm{C}$ & 5.89029858 & 1.03816672 & 1.92093133 \\
\hline $\mathrm{C}$ & 5.98920344 & 2.58967138 & 0.1860787 \\
\hline $\mathrm{N}$ & 5.16860323 & 1.90377155 & 1.09099608 \\
\hline 0 & 5.39040605 & 0.32796155 & 2.7567209 \\
\hline 0 & 5.61209152 & 3.47432009 & -0.5404855 \\
\hline C & 5.69121104 & -2.73718635 & -0.1122140 \\
\hline C & 5.77913606 & -1.52496787 & -2.3137096 \\
\hline $\mathrm{C}$ & 7.07034294 & -2.34567497 & 0.1351983 \\
\hline
\end{tabular}




$\begin{array}{rrrr}\mathrm{C} & 7.16381549 & -1.17341157 & -2.04124193 \\ \mathrm{C} & 7.76311957 & -1.56146834 & -0.81785602 \\ \mathrm{C} & 7.68733678 & -2.69287182 & 1.35339110 \\ \mathrm{C} & 7.87989391 & -0.38097166 & -2.96186107 \\ \mathrm{C} & 9.08930242 & -1.11342198 & -0.51863305 \\ \mathrm{C} & 8.96670782 & -2.25658646 & 1.64240020 \\ \mathrm{H} & 7.11703034 & -3.29020525 & 2.06521982 \\ \mathrm{C} & 9.16398081 & 0.04195818 & -2.67495425 \\ \mathrm{H} & 7.38573475 & -0.09778544 & -3.89195323 \\ \mathrm{C} & 9.67443519 & -1.45725724 & 0.72250515 \\ \mathrm{C} & 9.77629355 & -0.30597504 & -1.45478461 \\ \mathrm{H} & 9.44776545 & -2.50119027 & 2.59008482 \\ \mathrm{H} & 9.72392485 & 0.66767135 & -3.37091605 \\ \mathrm{C} & 10.99410932 & -0.94564362 & 1.06778556 \\ \mathrm{C} & 11.10280979 & 0.20455560 & -1.13635294 \\ \mathrm{~N} & 11.61272472 & -0.11974316 & 0.12421198 \\ \mathrm{O} & 11.55683778 & -1.19173575 & 2.13266336 \\ \mathrm{O} & 11.75687176 & 0.90940871 & -1.90272971 \\ \mathrm{C} & 12.92684603 & 0.41098828 & 0.46613372 \\ \mathrm{H} & 13.01815340 & 1.38392321 & -0.03230902 \\ \mathrm{H} & 12.94004075 & 0.55650024 & 1.55341171 \\ \mathrm{C} & 14.05498314 & -0.51257273 & 0.04025578 \\ \mathrm{H} & 14.04836048 & -0.65946386 & -1.04954760 \\ \mathrm{H} & 15.02710217 & -0.07963795 & 0.32102447 \\ \mathrm{H} & 13.96293736 & -1.49255914 & 0.53056675 \\ \mathrm{~N} & 5.12138440 & -2.25789151 & -1.30849050 \\ \mathrm{O} & 5.00995086 & -3.39822646 & 0.66045747 \\ \mathrm{O} & 5.16366119 & -1.18699036 & -3.31804452 \\ \mathrm{C} & 8.54338253 & 2.84489348 & -0.06789179 \\ \mathrm{H} & 9.35140280 & 2.20812897 & -0.45746675 \\ \mathrm{H} & 8.25772493 & 3.51847305 & -0.89029099 \\ \mathrm{C} & 9.06730378 & 3.62249832 & 1.13184538 \\ \mathrm{H} & 8.34719552 & 4.40868421 & 1.42332028 \\ \mathrm{H} & 9.99753187 & 4.14093154 & 0.85121944 \\ \mathrm{C} & 9.30243813 & 2.69575265 & 2.31794222 \\ \mathrm{H} & 9.73826862 & 3.24884139 & 3.16489906 \\ \mathrm{H} & 10.03318143 & 1.91378088 & 2.04394566 \\ \mathrm{C} & 7.99374888 & 2.05162840 & 2.75018244 \\ \mathrm{H} & 7.29742491 & 2.85060469 & 3.06342731 \\ \mathrm{H} & 8.13082817 & 1.39631548 & 3.62297167 \\ \mathrm{C} & 7.35731227 & 1.23055706 & 1.61523097 \\ \mathrm{H} & 7.84460509 & 0.24786098 & 1.56396881 \\ \mathrm{C} & 7.35730518 & 1.94658087 & 0.25755632 \\ \mathrm{H} & 7.29467382 & 1.17436851 & -0.53186034 \\ & & & \end{array}$

\section{Optimized single NDI acceptor (DA) structure of the singlet local excited state.}

$\begin{array}{llll}\mathrm{C} & -4.87608401 & 2.47722294 & -0.70176049 \\ \mathrm{C} & -5.85075533 & 3.41802296 & -0.91058488 \\ \mathrm{C} & -7.11370886 & 2.73882210 & -0.82112052 \\ \mathrm{~N} & -6.90142972 & 1.40526267 & -0.57854128 \\ \mathrm{C} & -5.54922082 & 1.22026690 & -0.51063564\end{array}$




\begin{tabular}{|c|c|c|c|}
\hline C & -5.82295283 & -3.45512105 & 0.31744628 \\
\hline C & -4.86405934 & -2.50653077 & 0.10968719 \\
\hline $\mathrm{C}$ & -5.54817818 & -1.24137387 & -0.01952753 \\
\hline $\mathrm{N}$ & -6.89652832 & -1.43466398 & 0.10002113 \\
\hline $\mathrm{C}$ & -7.09589257 & -2.77883635 & 0.31406601 \\
\hline $\mathrm{C}$ & -4.89822708 & -0.01501373 & -0.26733379 \\
\hline C & -11.81117243 & -2.42947964 & 0.73397139 \\
\hline C & -10.84067967 & -3.38985650 & 0.84648520 \\
\hline C & -9.58900518 & -2.75651243 & 0.53832155 \\
\hline $\mathrm{N}$ & -9.80388972 & -1.43291074 & 0.25033141 \\
\hline C & -11.14958715 & -1.20919348 & 0.35007433 \\
\hline $\mathrm{C}$ & -8.33396630 & -3.41499224 & 0.52782594 \\
\hline $\mathrm{C}$ & -10.89105898 & 3.39615326 & -0.79438847 \\
\hline $\mathrm{C}$ & -11.84529098 & 2.46895487 & -0.49385418 \\
\hline $\mathrm{C}$ & -11.16043841 & 1.22171667 & -0.24265310 \\
\hline $\mathrm{N}$ & -9.81013285 & 1.41132047 & -0.38415587 \\
\hline C & -9.61543256 & 2.72892301 & -0.72340581 \\
\hline C & -8.37815032 & 3.36665093 & -0.94136149 \\
\hline $\mathrm{C}$ & -11.80965348 & 0.01901509 & 0.09795206 \\
\hline $\mathrm{Zn}$ & -8.35371562 & -0.01288221 & -0.16058426 \\
\hline $\mathrm{H}$ & -3.80085971 & 2.63327286 & -0.66971450 \\
\hline $\mathrm{H}$ & -5.70013370 & 4.48094915 & -1.08203535 \\
\hline $\mathrm{H}$ & -5.65944934 & -4.52101343 & 0.45491363 \\
\hline $\mathrm{H}$ & -3.79034452 & -2.65699078 & 0.03079721 \\
\hline $\mathrm{H}$ & -12.87646471 & -2.56055329 & 0.90590752 \\
\hline $\mathrm{H}$ & -10.98701056 & -4.43027532 & 1.12568346 \\
\hline $\mathrm{H}$ & -11.05884415 & 4.44309308 & -1.03443744 \\
\hline $\mathrm{H}$ & -12.91845540 & 2.63596728 & -0.44875937 \\
\hline $\mathrm{C}$ & -13.30677826 & 0.04789590 & 0.27174383 \\
\hline $\mathrm{H}$ & -13.73075788 & -0.92229618 & -0.02346858 \\
\hline $\mathrm{H}$ & -13.74907307 & 0.77711079 & -0.42072028 \\
\hline $\mathrm{C}$ & -13.73759141 & 0.37896560 & 1.70160804 \\
\hline $\mathrm{H}$ & -14.83526578 & 0.38086532 & 1.79089762 \\
\hline $\mathrm{H}$ & -13.33796400 & -0.35655361 & 2.41614405 \\
\hline $\mathrm{H}$ & -13.36799936 & 1.37032500 & 2.00480598 \\
\hline $\mathrm{C}$ & -8.38893564 & 4.84581099 & -1.23273945 \\
\hline $\mathrm{H}$ & -7.53228901 & 5.09921788 & -1.87272899 \\
\hline $\mathrm{H}$ & -9.27828093 & 5.10074012 & -1.82519718 \\
\hline $\mathrm{C}$ & -8.35528314 & 5.70704270 & 0.03116729 \\
\hline $\mathrm{H}$ & -7.45582375 & 5.49827132 & 0.62962017 \\
\hline $\mathrm{H}$ & -8.35412615 & 6.77776523 & -0.22551423 \\
\hline $\mathrm{H}$ & -9.23058015 & 5.50858842 & 0.66778919 \\
\hline C & -8.32286395 & -4.88785989 & 0.84837187 \\
\hline $\mathrm{H}$ & -9.24449132 & -5.35315996 & 0.47241262 \\
\hline $\mathrm{H}$ & -7.50632609 & -5.38039402 & 0.30313331 \\
\hline $\mathrm{C}$ & -8.17910215 & -5.17259682 & 2.34445354 \\
\hline $\mathrm{H}$ & -9.00295339 & -4.71894939 & 2.91568190 \\
\hline $\mathrm{H}$ & -8.18481880 & -6.25644161 & 2.53810731 \\
\hline $\mathrm{H}$ & -7.23690942 & -4.76024796 & 2.73569283 \\
\hline $\mathrm{C}$ & -3.41687435 & -0.01804300 & -0.26550937 \\
\hline $\mathrm{C}$ & -2.68753379 & 0.32910015 & -1.41292078 \\
\hline C & -2.70528870 & -0.37569911 & 0.89005398 \\
\hline $\mathrm{C}$ & -1.29584458 & 0.30594040 & -1.4088095 \\
\hline $\mathrm{H}$ & -3.22369086 & 0.60186997 & -2.3248550 \\
\hline
\end{tabular}




\begin{tabular}{|c|c|c|c|}
\hline C & -1.31388340 & -0.38799252 & 0.89790863 \\
\hline $\mathrm{H}$ & -3.25635367 & -0.63704032 & 1.79638885 \\
\hline C & -0.59063261 & -0.05224371 & -0.25296282 \\
\hline $\mathrm{H}$ & -0.74355555 & 0.56450142 & -2.31560621 \\
\hline $\mathrm{H}$ & -0.77559922 & -0.66343998 & 1.80814089 \\
\hline C & 0.89484997 & -0.08687142 & -0.24422124 \\
\hline C & 1.61874898 & 0.96651587 & 0.35603779 \\
\hline C & 1.57137460 & -1.18095230 & -0.82543142 \\
\hline C & 3.05564564 & 0.91582727 & 0.37241462 \\
\hline $\mathrm{C}$ & 0.97563229 & 2.10321794 & 0.93541657 \\
\hline C & 3.00719321 & -1.21329630 & -0.79885343 \\
\hline C & 0.88414554 & -2.27959176 & -1.42865549 \\
\hline C & 3.77736587 & 1.99165403 & 0.98417458 \\
\hline C & 3.71142288 & -0.16715440 & -0.20661484 \\
\hline $\mathrm{C}$ & 1.70435865 & 3.11354506 & 1.50525042 \\
\hline $\mathrm{H}$ & -0.11377993 & 2.15799573 & 0.91432733 \\
\hline C & 3.67936194 & -2.33854059 & -1.37559604 \\
\hline C & 1.57105369 & -3.33068356 & -1.97650651 \\
\hline $\mathrm{H}$ & -0.20664627 & -2.27132230 & -1.44504466 \\
\hline C & 3.12342899 & 3.05858528 & 1.53584559 \\
\hline $\mathrm{H}$ & 4.79827365 & -0.19361281 & -0.18664833 \\
\hline $\mathrm{H}$ & 1.19545589 & 3.97578878 & 1.94055152 \\
\hline C & 2.99189543 & -3.36606086 & -1.95084571 \\
\hline $\mathrm{H}$ & 1.02626649 & -4.15970212 & -2.43235773 \\
\hline $\mathrm{H}$ & 3.69818811 & 3.86688262 & 1.99106830 \\
\hline $\mathrm{H}$ & 3.53493576 & -4.21019320 & -2.37851675 \\
\hline C & 5.95046889 & 1.12438574 & 1.83059994 \\
\hline C & 5.99360001 & 2.61741757 & 0.04843964 \\
\hline $\mathrm{N}$ & 5.19834805 & 1.93653319 & 0.97790596 \\
\hline O & 5.48560625 & 0.38784759 & 2.66353850 \\
\hline O & 5.58982332 & 3.44677793 & -0.72496413 \\
\hline C & 5.66932834 & -2.80140281 & -0.09558279 \\
\hline C & 5.79448992 & -1.62351632 & -2.29256802 \\
\hline C & 7.07082280 & -2.40301203 & 0.18172656 \\
\hline C & 7.20262386 & -1.25962613 & -1.99857055 \\
\hline C & 7.78284428 & -1.62865764 & -0.76290756 \\
\hline C & 7.65132235 & -2.73238471 & 1.39368669 \\
\hline C & 7.91631718 & -0.48935233 & -2.90095093 \\
\hline C & 9.09136346 & -1.18169140 & -0.44893257 \\
\hline C & 8.95033428 & -2.29084746 & 1.70293904 \\
\hline $\mathrm{H}$ & 7.07351679 & -3.31827289 & 2.11003793 \\
\hline C & 9.21616081 & -0.05169221 & -2.59057026 \\
\hline $\mathrm{H}$ & 7.44204630 & -0.20977718 & -3.84318685 \\
\hline C & 9.66048867 & -1.51884921 & 0.80011285 \\
\hline $\mathrm{C}$ & 9.79452434 & -0.38204202 & -1.37711991 \\
\hline $\mathrm{H}$ & 9.40829359 & -2.53179806 & 2.66365538 \\
\hline $\mathrm{H}$ & 9.77816435 & 0.57071219 & -3.28873128 \\
\hline $\mathrm{C}$ & 11.00484193 & -1.00599724 & 1.16033519 \\
\hline C & 11.14081503 & 0.13708096 & -1.03198557 \\
\hline $\mathrm{N}$ & 11.63473606 & -0.17844011 & 0.23191877 \\
\hline O & 11.53486343 & -1.27137841 & 2.22020752 \\
\hline O & 11.78255812 & 0.82547267 & -1.79992135 \\
\hline C & 12.95250520 & 0.35001511 & 0.58898462 \\
\hline $\mathrm{H}$ & 13.04728154 & 1.32413913 & 0.09415173 \\
\hline
\end{tabular}




$\begin{array}{rrrr}\mathrm{H} & 12.94923663 & 0.50045827 & 1.67536282 \\ \mathrm{C} & 14.08100537 & -0.57726394 & 0.17608571 \\ \mathrm{H} & 14.08813106 & -0.72948265 & -0.91278101 \\ \mathrm{H} & 15.04764758 & -0.13887575 & 0.46424097 \\ \mathrm{H} & 13.99015538 & -1.55437029 & 0.67187479 \\ \mathrm{~N} & 5.11114888 & -2.32591379 & -1.29244733 \\ \mathrm{O} & 5.01139062 & -3.45750609 & 0.67845534 \\ \mathrm{O} & 5.23535297 & -1.29270314 & -3.31399086 \\ \mathrm{C} & 8.53229770 & 2.99782826 & -0.19089885 \\ \mathrm{H} & 9.39283833 & 2.39701855 & -0.52625851 \\ \mathrm{H} & 8.22566811 & 3.60629551 & -1.05548609 \\ \mathrm{C} & 8.97116900 & 3.87396824 & 0.97459157 \\ \mathrm{H} & 8.19198275 & 4.62377446 & 1.19996649 \\ \mathrm{H} & 9.87151328 & 4.43922227 & 0.68825877 \\ \mathrm{C} & 9.23161803 & 3.03411995 & 2.21856537 \\ \mathrm{H} & 9.60043345 & 3.66391358 & 3.04303726 \\ \mathrm{H} & 10.02588355 & 2.29345079 & 2.01126293 \\ \mathrm{C} & 7.95830088 & 2.32323889 & 2.65237738 \\ \mathrm{H} & 7.19986865 & 3.08687275 & 2.90080883 \\ \mathrm{H} & 8.11121712 & 1.72996219 & 3.56591933 \\ \mathrm{C} & 7.41163454 & 1.39977309 & 1.54950005 \\ \mathrm{H} & 7.96595390 & 0.45151800 & 1.56349974 \\ \mathrm{C} & 7.39532286 & 2.04724039 & 0.15961733 \\ \mathrm{H} & 7.38553377 & 1.23977007 & -0.59514916\end{array}$

Optimized single NDI acceptor (DA) structure of the singlet charge separated excited state.

$\begin{array}{lr}\text { C } & -4.87191890 \\ \text { C } & -5.84055375 \\ \text { C } & -7.10559715 \\ \text { N } & -6.89890475 \\ \text { C } & -5.54921796 \\ \text { C } & -5.81135247 \\ \text { C } & -4.85400845 \\ \text { C } & -5.54244973 \\ \text { N } & -6.88473439 \\ \text { C } & -7.07959915 \\ \text { C } & -4.89569699 \\ \text { C } & -11.79296900 \\ \text { C } & -10.82633118 \\ \text { C } & -9.57354088 \\ \text { N } & -9.78563697 \\ \text { C } & -11.12966490 \\ \text { C } & -8.32286485 \\ \text { C } & -10.88130947 \\ \text { C } & -11.83552422 \\ \text { C } & -11.14650390 \\ \text { N } & -9.80221615 \\ \text { C } & -9.60998627 \\ \text { C } & -8.36452332 \\ \text { C } & -11.79047668 \\ \text { Zn } & -8.34091222 \\ \text { H } & -3.79731701 \\ \text { H } & -5.68844143 \\ & \end{array}$

$\begin{array}{rr}2.46512715 & -0.70592145 \\ 3.40010020 & -0.92602393 \\ 2.71606377 & -0.84419697 \\ 1.39094181 & -0.59760609 \\ 1.20863496 & -0.52022053 \\ -3.46104464 & 0.29894954 \\ -2.51310503 & 0.08549049 \\ -1.25500167 & -0.03409689 \\ -1.44283585 & 0.09235923 \\ -2.77883150 & 0.30695021 \\ -0.01887429 & -0.27600369 \\ -2.42278667 & 0.73619808 \\ -3.37727213 & 0.85637904 \\ -2.74165139 & 0.54225164 \\ -1.42845872 & 0.24636442 \\ -1.20599387 & 0.34519429 \\ -3.40707803 & 0.53453736 \\ 3.38371330 & -0.84819346 \\ 2.45874753 & -0.54189573 \\ 1.22428357 & -0.27102180 \\ 1.40933916 & -0.40679579 \\ 2.71532110 & -0.75875230 \\ 3.34780428 & -0.97484543 \\ 0.01522286 & 0.08786694 \\ -0.01845238 & -0.17353808 \\ 2.62023229 & -0.66238125 \\ 4.46187800 & -1.10049649\end{array}$




\begin{tabular}{|c|c|c|c|}
\hline $\mathrm{H}$ & -5.64794931 & -4.52697906 & 0.43092709 \\
\hline $\mathrm{H}$ & -3.78121225 & -2.66135207 & -0.00282517 \\
\hline $\mathrm{H}$ & -12.85785254 & -2.55623813 & 0.90597263 \\
\hline $\mathrm{H}$ & -10.97318740 & -4.41596469 & 1.13986052 \\
\hline $\mathrm{H}$ & -11.04881127 & 4.42602861 & -1.10551572 \\
\hline $\mathrm{H}$ & -12.90882342 & 2.62410294 & -0.50858210 \\
\hline C & -13.28395103 & 0.05282298 & 0.27264365 \\
\hline $\mathrm{H}$ & -13.71439005 & -0.92346018 & 0.01808703 \\
\hline $\mathrm{H}$ & -13.72726589 & 0.76178973 & -0.43783105 \\
\hline C & -13.68329544 & 0.43681412 & 1.69942520 \\
\hline $\mathrm{H}$ & -14.77866700 & 0.45693064 & 1.79534515 \\
\hline $\mathrm{H}$ & -13.28891763 & -0.28387941 & 2.43083722 \\
\hline $\mathrm{H}$ & -13.29816548 & 1.43165541 & 1.96739491 \\
\hline C & -8.37342890 & 4.82172994 & -1.28021210 \\
\hline $\mathrm{H}$ & -7.50753754 & 5.07301044 & -1.9056320 \\
\hline $\mathrm{H}$ & -9.25455140 & 5.06665473 & -1.88706939 \\
\hline C & -8.36422269 & 5.68349203 & -0.01517716 \\
\hline $\mathrm{H}$ & -7.47027801 & 5.48756961 & 0.59487206 \\
\hline $\mathrm{H}$ & -8.36801150 & 6.75016652 & -0.28248402 \\
\hline $\mathrm{H}$ & -9.24664597 & 5.48394495 & 0.61029014 \\
\hline $\mathrm{C}$ & -8.31226675 & -4.87354498 & 0.87192679 \\
\hline $\mathrm{H}$ & -9.23895904 & -5.34263119 & 0.5184145 \\
\hline $\mathrm{H}$ & -7.50292079 & -5.37284551 & 0.3244654 \\
\hline $\mathrm{C}$ & -8.14513739 & -5.12306621 & 2.3730331 \\
\hline $\mathrm{H}$ & -8.96290261 & -4.66422047 & 2.9481045 \\
\hline $\mathrm{H}$ & -8.14474421 & -6.20292760 & 2.5806014 \\
\hline $\mathrm{H}$ & -7.19807611 & -4.70359833 & 2.7428584 \\
\hline $\mathrm{C}$ & -3.41565509 & -0.02071602 & -0.2649699 \\
\hline C & -2.68634656 & 0.33037909 & -1.4096790 \\
\hline C & -2.72127384 & -0.38477369 & 0.8978455 \\
\hline C & -1.29542170 & 0.30290138 & -1.3931788 \\
\hline $\mathrm{H}$ & -3.21449920 & 0.60237791 & -2.3264503 \\
\hline C & -1.33093278 & -0.38737767 & 0.9158107 \\
\hline $\mathrm{H}$ & -3.27767117 & -0.65172498 & 1.7992537 \\
\hline $\mathrm{C}$ & -0.59974809 & -0.05175744 & -0.2305993 \\
\hline $\mathrm{H}$ & -0.73417243 & 0.55850275 & -2.2946049 \\
\hline $\mathrm{H}$ & -0.79718462 & -0.66070787 & 1.8286823 \\
\hline C & 0.88554399 & -0.08296407 & -0.2112699 \\
\hline C & 1.59709613 & 0.96301173 & 0.4139739 \\
\hline C & 1.56533118 & -1.16485734 & -0.8097393 \\
\hline C & 3.03369088 & 0.91544661 & 0.43751263 \\
\hline C & 0.95074434 & 2.08992185 & 1.00974357 \\
\hline C & 3.00103308 & -1.18801683 & -0.78185139 \\
\hline C & 0.88903231 & -2.25900231 & -1.4335862 \\
\hline C & 3.75217035 & 1.97672497 & 1.08015671 \\
\hline C & 3.69641308 & -0.14974894 & -0.16603668 \\
\hline $\mathrm{C}$ & 1.67628798 & 3.08932617 & 1.60283439 \\
\hline $\mathrm{H}$ & -0.13838313 & 2.15053640 & 0.98410643 \\
\hline C & 3.69545706 & -2.28995589 & -1.37988858 \\
\hline C & 1.59220580 & -3.29053435 & -1.99737217 \\
\hline $\mathrm{H}$ & -0.20168622 & -2.26754663 & -1.45451083 \\
\hline $\mathrm{C}$ & 3.09529155 & 3.03338424 & 1.64713213 \\
\hline $\mathrm{H}$ & 4.78385775 & -0.17677948 & -0.15380978 \\
\hline $\mathrm{H}$ & 1.16379304 & 3.94358327 & 2.0502294 \\
\hline
\end{tabular}




\begin{tabular}{|c|c|c|c|}
\hline C & 3.01363389 & -3.31103654 & -1.97315432 \\
\hline $\mathrm{H}$ & 1.05763363 & -4.11777968 & -2.46946578 \\
\hline $\mathrm{H}$ & 3.66720775 & 3.82934933 & 2.12643077 \\
\hline $\mathrm{H}$ & 3.56584154 & -4.13917349 & -2.41918152 \\
\hline $\mathrm{C}$ & 5.89029392 & 1.03837641 & 1.92078268 \\
\hline $\mathrm{C}$ & 5.98943157 & 2.58977862 & 0.18585124 \\
\hline $\mathrm{N}$ & 5.16871118 & 1.90403113 & 1.09076080 \\
\hline 0 & 5.39030275 & 0.32831313 & 2.75662802 \\
\hline 0 & 5.61247438 & 3.47441576 & -0.54082790 \\
\hline $\mathrm{C}$ & 5.69103916 & -2.73706286 & -0.11166600 \\
\hline $\mathrm{C}$ & 5.77892994 & -1.52513973 & -2.31333870 \\
\hline $\mathrm{C}$ & 7.07022652 & -2.34558469 & 0.13560527 \\
\hline C & 7.16364641 & -1.17368979 & -2.04102118 \\
\hline $\mathrm{C}$ & 7.76298457 & -1.56157209 & -0.81759424 \\
\hline $\mathrm{C}$ & 7.68724539 & -2.69260479 & 1.3538378 \\
\hline $\mathrm{C}$ & 7.87974759 & -0.38147806 & -2.96183643 \\
\hline $\mathrm{C}$ & 9.08919568 & -1.11349551 & -0.51849165 \\
\hline $\mathrm{C}$ & 8.96662686 & -2.25628630 & 1.64274382 \\
\hline $\mathrm{H}$ & 7.11693710 & -3.28979332 & 2.06578528 \\
\hline C & 9.16387297 & 0.04141435 & -2.6750805 \\
\hline $\mathrm{H}$ & 7.38554800 & -0.09841147 & -3.8919413 \\
\hline C & 9.67433824 & -1.45709669 & 0.7226994 \\
\hline $\mathrm{C}$ & 9.77619237 & -0.30628103 & -1.4548313 \\
\hline $\mathrm{H}$ & 9.44769639 & -2.50070711 & 2.5904693 \\
\hline $\mathrm{H}$ & 9.72385394 & 0.66692272 & -3.37119564 \\
\hline C & 10.99407138 & -0.94552535 & 1.0678239 \\
\hline C & 11.10280153 & 0.20411774 & -1.1366096 \\
\hline $\mathrm{N}$ & 11.61269292 & -0.11985735 & 0.1240513 \\
\hline 0 & 11.55688705 & -1.19157999 & 2.1326637 \\
\hline 0 & 11.75698029 & 0.90861202 & -1.9032207 \\
\hline $\mathrm{C}$ & 12.92695446 & 0.41067824 & 0.4656698 \\
\hline $\mathrm{H}$ & 13.01825188 & 1.38367754 & -0.0326545 \\
\hline $\mathrm{H}$ & 12.94043435 & 0.55603726 & 1.5529611 \\
\hline $\mathrm{C}$ & 14.05488328 & -0.51294827 & 0.0394003 \\
\hline $\mathrm{H}$ & 14.04800720 & -0.65967091 & -1.0504194 \\
\hline $\mathrm{H}$ & 15.02709952 & -0.08014391 & 0.32004542 \\
\hline $\mathrm{H}$ & 13.96283650 & -1.49299613 & 0.52959350 \\
\hline $\mathrm{N}$ & 5.12122113 & -2.25806865 & -1.30803616 \\
\hline 0 & 5.00980419 & -3.39791341 & 0.66118249 \\
\hline 0 & 5.16335902 & -1.18725246 & -3.31763260 \\
\hline $\mathrm{C}$ & 8.54368136 & 2.84464428 & -0.06800833 \\
\hline $\mathrm{H}$ & 9.35162325 & 2.20762470 & -0.45735181 \\
\hline $\mathrm{H}$ & 8.25821902 & 3.51805261 & -0.8906187 \\
\hline $\mathrm{C}$ & 9.06757313 & 3.62243920 & 1.1316022 \\
\hline $\mathrm{H}$ & 8.34754197 & 4.40879433 & 1.42281152 \\
\hline $\mathrm{H}$ & 9.99791039 & 4.14066877 & 0.85096501 \\
\hline $\mathrm{C}$ & 9.30246724 & 2.69589543 & 2.31791281 \\
\hline $\mathrm{H}$ & 9.73817305 & 3.24911449 & 3.16484883 \\
\hline $\mathrm{H}$ & 10.03323721 & 1.91386604 & 2.04416301 \\
\hline $\mathrm{C}$ & 7.99369866 & 2.05187941 & 2.7500658 \\
\hline $\mathrm{H}$ & 7.29732410 & 2.85091568 & 3.0630548 \\
\hline $\mathrm{H}$ & 8.13062846 & 1.39673797 & 3.62300375 \\
\hline $\mathrm{C}$ & 7.35733984 & 1.23065615 & 1.6151690 \\
\hline $\mathrm{H}$ & 7.84457138 & 0.24791076 & 1.5640962 \\
\hline
\end{tabular}




$\begin{array}{lllr}\mathrm{C} & 7.35746144 & 1.94652981 & 0.25743991 \\ \mathrm{H} & 7.29477892 & 1.17430419 & -0.53196484\end{array}$

Optimized double NDI acceptor $\left(\mathrm{DA}_{2}\right)$ structure on the ground state.

\begin{tabular}{|c|c|c|c|}
\hline C & -6.24743649 & 2.39749223 & -0.88758264 \\
\hline $\mathrm{C}$ & -7.25338027 & 3.25283152 & -1.22299335 \\
\hline C & -8.49341407 & 2.52256022 & -1.08946585 \\
\hline $\mathrm{N}$ & -8.22221951 & 1.24191243 & -0.68700966 \\
\hline $\mathrm{C}$ & -6.86601982 & 1.13366870 & -0.56510148 \\
\hline $\mathrm{C}$ & -6.90065871 & -3.40863470 & 0.78417935 \\
\hline $\mathrm{C}$ & -5.99075780 & -2.43060549 & 0.51677656 \\
\hline $\mathrm{C}$ & -6.74138318 & -1.24538100 & 0.17715082 \\
\hline $\mathrm{N}$ & -8.07876263 & -1.51691200 & 0.23415668 \\
\hline $\mathrm{C}$ & -8.21219901 & -2.82814251 & 0.60755054 \\
\hline $\mathrm{C}$ & -6.15773835 & -0.01573401 & -0.17449139 \\
\hline $\mathrm{C}$ & -12.94571813 & -2.76692787 & 0.62542054 \\
\hline $\mathrm{C}$ & -11.93590464 & -3.63841162 & 0.90169159 \\
\hline $\mathrm{C}$ & -10.69642140 & -2.94380638 & 0.64597942 \\
\hline $\mathrm{N}$ & -10.97121436 & -1.67041465 & 0.22775548 \\
\hline $\mathrm{C}$ & -12.33260718 & -1.53239613 & 0.19593311 \\
\hline $\mathrm{C}$ & -9.42054716 & -3.51752804 & 0.80769635 \\
\hline $\mathrm{C}$ & -12.28369956 & 2.96299419 & -1.29641537 \\
\hline $\mathrm{C}$ & -13.19726217 & 2.00435579 & -0.97732201 \\
\hline $\mathrm{C}$ & -12.45664977 & 0.82556911 & -0.59469654 \\
\hline $\mathrm{N}$ & -11.11584893 & 1.08746660 & -0.68251148 \\
\hline $\mathrm{C}$ & -10.97637230 & 2.38038872 & -1.10823832 \\
\hline $\mathrm{C}$ & -9.76692870 & 3.06988471 & -1.32060783 \\
\hline $\mathrm{C}$ & -13.04955975 & -0.38625134 & -0.19466290 \\
\hline $\mathrm{Zn}$ & -9.59632090 & -0.21633437 & -0.23295590 \\
\hline $\mathrm{H}$ & -5.18076186 & 2.60634118 & -0.85545930 \\
\hline $\mathrm{H}$ & -7.14805251 & 4.29457827 & -1.51605064 \\
\hline $\mathrm{H}$ & -6.68360624 & -4.43380133 & 1.07390514 \\
\hline $\mathrm{H}$ & -4.90636575 & -2.50865695 & 0.53674541 \\
\hline $\mathrm{H}$ & -14.01156615 & -2.96207191 & 0.71386329 \\
\hline $\mathrm{H}$ & -12.04133310 & -4.66169438 & 1.25342506 \\
\hline $\mathrm{H}$ & -12.49649533 & 3.97734937 & -1.62463338 \\
\hline $\mathrm{H}$ & -14.27925316 & 2.10755524 & -1.00163385 \\
\hline C & -14.55893493 & -0.43798283 & -0.11245029 \\
\hline $\mathrm{H}$ & -14.90789436 & -1.45352569 & -0.34262099 \\
\hline $\mathrm{H}$ & -14.99427291 & 0.19492717 & -0.89729920 \\
\hline $\mathrm{C}$ & -15.09993352 & -0.00788468 & 1.24988573 \\
\hline $\mathrm{H}$ & -16.19975096 & -0.05830283 & 1.27125278 \\
\hline $\mathrm{H}$ & -14.71340473 & -0.65502866 & 2.05187598 \\
\hline $\mathrm{H}$ & -14.80138812 & 1.02471670 & 1.48651327 \\
\hline $\mathrm{C}$ & -9.84624491 & 4.51948724 & -1.74487225 \\
\hline $\mathrm{H}$ & -8.97507829 & 4.77019226 & -2.36508118 \\
\hline $\mathrm{H}$ & -10.71344064 & 4.66442670 & -2.40353696 \\
\hline $\mathrm{C}$ & -9.93094052 & 5.48838840 & -0.56686107 \\
\hline $\mathrm{H}$ & -9.05026565 & 5.39496777 & 0.08659120 \\
\hline $\mathrm{H}$ & -9.98647942 & 6.53090992 & -0.91704231 \\
\hline $\mathrm{H}$ & -10.82086193 & 5.28769938 & 0.04881875 \\
\hline $\mathrm{C}$ & -9.34536393 & -4.94489067 & 1.301657 \\
\hline
\end{tabular}




\begin{tabular}{|c|c|c|c|}
\hline $\mathrm{H}$ & -10.20010648 & -5.51638241 & 0.91546790 \\
\hline $\mathrm{H}$ & -8.46040426 & -5.43696836 & 0.87602990 \\
\hline $\mathrm{C}$ & -9.30434820 & -5.05082941 & 2.82506957 \\
\hline $\mathrm{H}$ & -10.20193727 & -4.60297059 & 3.27802161 \\
\hline $\mathrm{H}$ & -9.24913496 & -6.10275319 & 3.14596104 \\
\hline $\mathrm{H}$ & -8.42968610 & -4.52316889 & 3.23480023 \\
\hline C & -4.67239724 & 0.07391399 & -0.12703995 \\
\hline C & -3.92336933 & 0.23039938 & -1.30035397 \\
\hline C & -3.99003942 & -0.00166453 & 1.09409763 \\
\hline $\mathrm{C}$ & -2.53360806 & 0.30436308 & -1.25515204 \\
\hline $\mathrm{H}$ & -4.44035620 & 0.28652410 & -2.26121758 \\
\hline $\mathrm{C}$ & -2.60070523 & 0.07462788 & 1.14125261 \\
\hline $\mathrm{H}$ & -4.56035870 & -0.11863306 & 2.01860511 \\
\hline $\mathrm{C}$ & -1.85351684 & 0.22631866 & -0.03345376 \\
\hline $\mathrm{H}$ & -1.96351721 & 0.42274275 & -2.17986176 \\
\hline $\mathrm{H}$ & -2.08342394 & 0.01364021 & 2.10187742 \\
\hline C & -0.37091781 & 0.29700704 & 0.01693495 \\
\hline C & 0.26126303 & 1.44342360 & 0.54764379 \\
\hline $\mathrm{C}$ & 0.40018794 & -0.78629014 & -0.46200420 \\
\hline $\mathrm{C}$ & 1.69485748 & 1.48490390 & 0.61718352 \\
\hline $\mathrm{C}$ & -0.46268889 & 2.58458300 & 1.01439683 \\
\hline C & 1.83408579 & -0.70009554 & -0.42392284 \\
\hline C & -0.18511729 & -1.98540837 & -0.97351934 \\
\hline C & 2.32737451 & 2.63178904 & 1.19144131 \\
\hline C & 2.44413803 & 0.42150500 & 0.12405506 \\
\hline $\mathrm{C}$ & 0.18719639 & 3.67125491 & 1.53826968 \\
\hline $\mathrm{H}$ & -1.55129468 & 2.58178602 & 0.94534226 \\
\hline $\mathrm{C}$ & 2.60762112 & -1.78103718 & -0.94596039 \\
\hline $\mathrm{C}$ & 0.59510420 & -3.00949644 & -1.44213162 \\
\hline $\mathrm{H}$ & -1.27178305 & -2.07878951 & -0.98405965 \\
\hline $\mathrm{C}$ & 1.60522489 & 3.69666206 & 1.64166762 \\
\hline $\mathrm{H}$ & 3.53056824 & 0.45949350 & 0.16438058 \\
\hline $\mathrm{H}$ & -0.38643183 & 4.53258576 & 1.88614036 \\
\hline $\mathrm{C}$ & 2.01209232 & -2.90521846 & -1.44170242 \\
\hline $\mathrm{H}$ & 0.12867109 & -3.91887940 & -1.82599310 \\
\hline $\mathrm{H}$ & 2.11611848 & 4.55666599 & 2.07732671 \\
\hline $\mathrm{H}$ & 2.62668112 & -3.71625282 & -1.83631334 \\
\hline $\mathrm{C}$ & 4.74127739 & -2.02838089 & 0.18620523 \\
\hline $\mathrm{C}$ & 4.59812547 & -1.04859287 & -2.10261186 \\
\hline $\mathrm{C}$ & 6.22048078 & -1.92993616 & 0.11831637 \\
\hline C & 6.07776322 & -0.96165381 & -2.14245528 \\
\hline C & 6.84098027 & -1.43543246 & -1.05192750 \\
\hline $\mathrm{C}$ & 6.99148844 & -2.36653558 & 1.18144291 \\
\hline $\mathrm{C}$ & 6.70915671 & -0.46094749 & -3.26799785 \\
\hline $\mathrm{C}$ & 8.25589244 & -1.43736780 & -1.14364287 \\
\hline $\mathrm{C}$ & 8.39530831 & -2.37555505 & 1.08722954 \\
\hline $\mathrm{H}$ & 6.49016179 & -2.73041083 & 2.07984350 \\
\hline $\mathrm{C}$ & 8.11253573 & -0.46078621 & -3.35614017 \\
\hline $\mathrm{H}$ & 6.09689456 & -0.09171284 & -4.09251198 \\
\hline $\mathrm{C}$ & 9.02161186 & -1.93972747 & -0.06737067 \\
\hline $\mathrm{C}$ & 8.87868829 & -0.96136106 & -2.31847475 \\
\hline $\mathrm{H}$ & 9.00912476 & -2.74612485 & 1.91007517 \\
\hline $\mathrm{H}$ & 8.61754434 & -0.08926256 & -4.24939666 \\
\hline C & 10.49694131 & -2.04252017 & -0.196658 \\
\hline
\end{tabular}




$\begin{array}{rrrr}\mathrm{C} & 10.35412156 & -1.03724613 & -2.45498206 \\ \mathrm{~N} & 11.06277250 & -1.56786705 & -1.37637442 \\ \mathrm{O} & 11.18472034 & -2.52936619 & 0.67895137 \\ \mathrm{O} & 10.92764608 & -0.68432822 & -3.46398556 \\ \mathrm{C} & 12.50978948 & -1.71141228 & -1.54213441 \\ \mathrm{H} & 12.85215351 & -0.83880189 & -2.11210641 \\ \mathrm{H} & 12.94846070 & -1.67824587 & -0.53863619 \\ \mathrm{C} & 12.88408687 & -3.00384552 & -2.24509591 \\ \mathrm{H} & 12.44582143 & -3.04851087 & -3.25216195 \\ \mathrm{H} & 13.97721292 & -3.06883296 & -2.34706328 \\ \mathrm{H} & 12.54451992 & -3.87715934 & -1.66940525 \\ \mathrm{~N} & 4.03821537 & -1.64847083 & -0.96450194 \\ \mathrm{O} & 4.15700050 & -2.43353869 & 1.16640537 \\ \mathrm{O} & 3.89542137 & -0.65236202 & -3.00490603 \\ \mathrm{C} & 4.47901173 & 2.87729846 & 0.12571624 \\ \mathrm{C} & 4.27065159 & 1.94254072 & 2.43129274 \\ \mathrm{C} & 5.90570705 & 2.47304642 & 0.10868849 \\ \mathrm{C} & 5.70440872 & 1.57014562 & 2.39369829 \\ \mathrm{C} & 6.47229867 & 1.83905002 & 1.23833931 \\ \mathrm{C} & 6.67354091 & 2.70262020 & -1.01979720 \\ \mathrm{C} & 6.28084218 & 0.94167290 & 3.48389175 \\ \mathrm{C} & 7.84167032 & 1.47420317 & 1.21570130 \\ \mathrm{C} & 8.03153975 & 2.33936461 & -1.04137204 \\ \mathrm{H} & 6.20736507 & 3.17905903 & -1.88383051 \\ \mathrm{C} & 7.64087232 & 0.58774984 & 3.46295856 \\ \mathrm{H} & 5.66131183 & 0.73501360 & 4.35813578 \\ \mathrm{C} & 8.61449401 & 1.74812571 & 0.06581185 \\ \mathrm{C} & 8.41615520 & 0.85717631 & 2.34879204 \\ \mathrm{H} & 8.64879845 & 2.53060864 & -1.92049147 \\ \mathrm{H} & 8.10724030 & 0.10608742 & 4.32400591 \\ \mathrm{C} & 10.06189581 & 1.42819516 & 0.05725576 \\ \mathrm{C} & 9.86130778 & 0.52737318 & 2.35471576 \\ \mathrm{~N} & 3.75703899 & 2.57246229 & 1.28762721 \\ \mathrm{~N} & 10.58984282 & 0.84920092 & 1.20917876 \\ \mathrm{O} & 3.55885053 & 1.70266875 & 3.38023692 \\ \mathrm{O} & 3.94080007 & 3.41388675 & -0.81654571 \\ \mathrm{O} & 10.76475924 & 1.66231454 & -0.90625736 \\ \mathrm{O} & 10.40317232 & 0.01388055 & 3.31177193 \\ \mathrm{C} & 12.03645245 & 0.62930973 & 1.26083954 \\ \mathrm{H} & 12.37073689 & 0.51012475 & 0.22516349 \\ \mathrm{H} & 12.19973834 & -0.31199236 & 1.79854206 \\ \mathrm{C} & 12.76484752 & 1.78251367 & 1.92702111 \\ \mathrm{H} & 13.84546228 & 1.57923452 & 1.94264576 \\ \mathrm{H} & 12.60391985 & 2.72155392 & 1.37743565 \\ \mathrm{H} & 12.43026134 & 1.91625502 & 2.96555084 \\ & & & \end{array}$

\section{Optimized double NDI acceptor $\left(\mathrm{DA}_{2}\right)$ structure of the triplet local excited state.}

$\begin{array}{llll}\text { C } & -6.256238 & 2.394220 & -0.806962 \\ \text { C } & -7.283483 & 3.266701 & -1.152917 \\ \text { C } & -8.487496 & 2.528942 & -1.070265\end{array}$




\begin{tabular}{|c|c|c|c|}
\hline $\mathrm{N}$ & -8.223780 & 1.238701 & -0.693175 \\
\hline $\mathrm{C}$ & -6.864179 & 1.145638 & -0.54013 \\
\hline C & -6.878041 & -3.449603 & 0.73515 \\
\hline C & -5.969129 & -2.471221 & 0.48045 \\
\hline C & -6.720371 & -1.271579 & 0.17874 \\
\hline $\mathrm{N}$ & -8.059522 & -1.536723 & 0.24713 \\
\hline C & -8.192114 & -2.857161 & 0.58980 \\
\hline C & -6.147414 & -0.038479 & -0.15817 \\
\hline $\mathrm{C}$ & -12.949369 & -2.724098 & 0.6784 \\
\hline $\mathrm{C}$ & -11.919247 & -3.617428 & $0.9508^{\circ}$ \\
\hline $\mathrm{C}$ & -10.710378 & -2.944075 & 0.6588 \\
\hline $\mathrm{N}$ & -10.977780 & -1.670924 & 0.22561 \\
\hline $\mathrm{C}$ & -12.341627 & -1.529917 & 0.22535 \\
\hline C & -9.401214 & -3.533146 & 0.79012 \\
\hline $\mathrm{C}$ & -12.316716 & 2.973885 & -1.34910 \\
\hline C & -13.228368 & 2.021839 & -1.02054 \\
\hline C & -12.485950 & 0.848141 & -0.60762 \\
\hline $\mathrm{N}$ & -11.144551 & 1.110023 & -0.684 \\
\hline C & -11.005595 & 2.397288 & -1.1350 \\
\hline $\mathrm{C}$ & -9.797772 & 3.074687 & -1.3359 \\
\hline $\mathrm{C}$ & -13.067610 & -0.356141 & -0.18992 \\
\hline $\mathrm{Zn}$ & -9.600225 & -0.218003 & -0.235 \\
\hline $\mathrm{H}$ & -5.194059 & 2.616902 & -0.743 \\
\hline $\mathrm{H}$ & -7.177731 & 4.315527 & -1.4187 \\
\hline $\mathrm{H}$ & -6.663086 & -4.482875 & 0.9961 \\
\hline $\mathrm{H}$ & -4.885046 & -2.554456 & 0.4818 \\
\hline $\mathrm{H}$ & -14.013532 & -2.913230 & 0.79432 \\
\hline $\mathrm{H}$ & -12.028097 & -4.634207 & 1.3196 \\
\hline $\mathrm{H}$ & -12.527384 & 3.981607 & -1.69839 \\
\hline $\mathrm{H}$ & -14.310396 & 2.120805 & -1.05634 \\
\hline C & -14.569719 & -0.420302 & -0.09487 \\
\hline $\mathrm{H}$ & -14.906631 & -1.438892 & -0.33067 \\
\hline $\mathrm{H}$ & -15.021654 & 0.222383 & -0.86181 \\
\hline $\mathrm{C}$ & -15.092178 & -0.012483 & 1.28368 \\
\hline $\mathrm{H}$ & -16.189514 & -0.090814 & 1.32294 \\
\hline $\mathrm{H}$ & -14.675205 & -0.655442 & 2.07284 \\
\hline $\mathrm{H}$ & -14.815599 & 1.026403 & 1.51911 \\
\hline C & -9.855372 & 4.514647 & -1.77464 \\
\hline $\mathrm{H}$ & -8.976660 & 4.739600 & -2.39472 \\
\hline $\mathrm{H}$ & -10.724037 & 4.671346 & -2.42837 \\
\hline C & -9.919388 & 5.492307 & $-0.5997 \varepsilon$ \\
\hline $\mathrm{H}$ & -9.050343 & 5.374182 & 0.06407 \\
\hline $\mathrm{H}$ & -9.935606 & 6.532460 & -0.95974 \\
\hline $\mathrm{H}$ & -10.824385 & 5.325676 & 0.00379 \\
\hline C & -9.343661 & -4.964004 & 1.25753 \\
\hline $\mathrm{H}$ & -10.206467 & -5.513164 & 0.85655 \\
\hline $\mathrm{H}$ & -8.458587 & -5.459451 & 0.83652 \\
\hline C & -9.317929 & -5.087927 & 2.78203 \\
\hline $\mathrm{H}$ & -10.204801 & -4.620104 & 3.23438 \\
\hline $\mathrm{H}$ & -9.296597 & -6.145701 & 3.08611 \\
\hline $\mathrm{H}$ & -8.429226 & -4.593877 & 3.20299 \\
\hline C & -4.667007 & 0.059281 & $-0.1152 \varepsilon$ \\
\hline C & -3.927726 & 0.282188 & -1.28480 \\
\hline $\mathrm{C}$ & -3.978245 & -0.074187 & 1.09740 \\
\hline
\end{tabular}




\begin{tabular}{|c|c|c|c|}
\hline $\mathrm{C}$ & -2.538384 & 0.353560 & -1.24409 \\
\hline $\mathrm{H}$ & -4.451512 & 0.390155 & -2.23716 \\
\hline$c$ & -2.589182 & 0.009573 & 1.13994 \\
\hline $\mathrm{H}$ & -4.542190 & -0.239014 & 2.01843 \\
\hline C & -1.851075 & 0.219517 & -0.0308 \\
\hline $\mathrm{H}$ & -1.973702 & 0.516978 & -2.16517 \\
\hline $\mathrm{H}$ & -2.065465 & -0.092856 & 2.09345 \\
\hline $\mathrm{C}$ & -0.368226 & 0.293436 & 0.015 \\
\hline C & 0.261860 & 1.434833 & 0.5579 \\
\hline C & 0.403357 & -0.782836 & -0.47701 \\
\hline C & 1.695463 & 1.478844 & 0.62498 \\
\hline C & -0.464975 & 2.567387 & $1.0406 \varepsilon$ \\
\hline $\mathrm{C}$ & 1.837118 & -0.696207 & -0.43685 \\
\hline C & -0.181877 & -1.974642 & -1.00512 \\
\hline $\mathrm{C}$ & 2.326000 & 2.621546 & 1.2095 \\
\hline C & 2.445915 & 0.421101 & 0.1212 \\
\hline $\mathrm{C}$ & 0.183141 & 3.650225 & 1.5744 \\
\hline $\mathrm{H}$ & -1.553931 & 2.560824 & 0.975 \\
\hline C & 2.611162 & -1.772244 & $-0.968 c$ \\
\hline C & 0.598884 & -2.993517 & -1.4840 \\
\hline $\mathrm{H}$ & -1.268754 & -2.065849 & $-1.0207^{-}$ \\
\hline C & 1.601524 & 3.679186 & 1.6730 \\
\hline $\mathrm{H}$ & 3.532352 & 0.460446 & 0.1612 \\
\hline $\mathrm{H}$ & -0.392085 & 4.505429 & 1.9345 \\
\hline C & 2.016019 & -2.890570 & -1.477 \\
\hline $\mathrm{H}$ & 0.133053 & -3.897474 & -1.881 \\
\hline $\mathrm{H}$ & 2.110890 & 4.536034 & 2.116 \\
\hline $\mathrm{H}$ & 2.631119 & -3.697808 & -1.87 \\
\hline C & 4.740683 & -2.028106 & 0.16 \\
\hline C & 4.605845 & -1.034592 & -2.11 \\
\hline $\mathrm{C}$ & 6.220084 & -1.928059 & 0 . \\
\hline C & 6.085664 & -0.949758 & -2 . \\
\hline C & 6.844847 & -1.428802 & -1 \\
\hline C & 6.987292 & -2.368413 & 1.1725 \\
\hline C & 6.721148 & -0.445525 & -3.27053 \\
\hline C & 8.260072 & -1.431374 & -1.14489 \\
\hline C & 8.391466 & -2.376669 & 1.08346 \\
\hline $\mathrm{H}$ & 6.482763 & -2.735907 & 2.06763 \\
\hline C & 8.124761 & -0.447401 & -3.35465 \\
\hline $\mathrm{H}$ & 6.111870 & -0.072128 & -4.09539 \\
\hline C & 9.021912 & -1.937355 & -0.0675 \\
\hline C & 8.887049 & -0.952578 & -2.31638 \\
\hline $\mathrm{H}$ & 9.002349 & -2.749806 & 1.90733 \\
\hline $\mathrm{H}$ & 8.632993 & -0.073808 & -4.24521 \\
\hline C & 10.497649 & -2.040283 & -0.1920 \\
\hline C & 10.362672 & -1.031434 & -2.44933 \\
\hline $\mathrm{N}$ & 11.067535 & -1.564503 & -1.36941 \\
\hline O & 11.182417 & -2.528527 & 0.6851 \\
\hline O & 10.939388 & -0.678921 & -3.45662 \\
\hline C & 12.514573 & -1.711840 & -1.5319 \\
\hline $\mathrm{H}$ & 12.860407 & -0.840561 & -2.10182 \\
\hline $\mathrm{H}$ & 12.951277 & -1.679157 & -0.52756 \\
\hline C & 12.886913 & -3.005676 & -2.2333 \\
\hline $\mathrm{H}$ & 12.450565 & -3.049795 & -3.24128 \\
\hline
\end{tabular}

S38 


$\begin{array}{rrrr}\mathrm{H} & 13.980072 & -3.073585 & -2.333060 \\ \mathrm{H} & 12.543910 & -3.877758 & -1.657837 \\ \mathrm{~N} & 4.041832 & -1.640808 & -0.981123 \\ \mathrm{O} & 4.152774 & -2.440104 & 1.144898 \\ \mathrm{O} & 3.906231 & -0.631801 & -3.015596 \\ \mathrm{C} & 4.475551 & 2.877888 & 0.141773 \\ \mathrm{C} & 4.272868 & 1.930443 & 2.442692 \\ \mathrm{C} & 5.902702 & 2.475320 & 0.119649 \\ \mathrm{C} & 5.707619 & 1.562438 & 2.401230 \\ \mathrm{C} & 6.472718 & 1.837647 & 1.245496 \\ \mathrm{C} & 6.667703 & 2.710344 & -1.009637 \\ \mathrm{C} & 6.287491 & 0.931187 & 3.487992 \\ \mathrm{C} & 7.842655 & 1.475264 & 1.218667 \\ \mathrm{C} & 8.026093 & 2.348738 & -1.035723 \\ \mathrm{H} & 6.199019 & 3.189642 & -1.870734 \\ \mathrm{C} & 7.648264 & 0.580495 & 3.463234 \\ \mathrm{H} & 5.669995 & 0.719793 & 4.362551 \\ \mathrm{C} & 8.612435 & 1.754295 & 0.067955 \\ \mathrm{C} & 8.420677 & 0.855455 & 2.348442 \\ \mathrm{H} & 8.641037 & 2.543914 & -1.915601 \\ \mathrm{H} & 8.117467 & 0.096921 & 4.321666 \\ \mathrm{C} & 10.060217 & 1.436199 & 0.054921 \\ \mathrm{C} & 9.866579 & 0.528949 & 2.350512 \\ \mathrm{~N} & 3.755950 & 2.564945 & 1.303097 \\ \mathrm{~N} & 10.591790 & 0.854857 & 1.204024 \\ \mathrm{O} & 3.563104 & 1.683336 & 3.391322 \\ \mathrm{O} & 3.934849 & 3.419404 & -0.796195 \\ \mathrm{O} & 10.760458 & 1.673850 & -0.909618 \\ \mathrm{O} & 10.411703 & 0.014643 & 3.305252 \\ \mathrm{C} & 12.038945 & 0.637770 & 1.251838 \\ \mathrm{H} & 12.371041 & 0.521387 & 0.215142 \\ \mathrm{H} & 12.205304 & -0.304222 & 1.787417 \\ \mathrm{C} & 12.766591 & 1.791044 & 1.918724 \\ \mathrm{H} & 13.847661 & 1.589972 & 1.931278 \\ \mathrm{H} & 12.602386 & 2.730918 & 1.371537 \\ \mathrm{H} & 12.434258 & 1.921853 & 2.958352 \\ & & & \end{array}$

\section{Optimized double NDI acceptor $\left(\mathrm{DA}_{2}\right)$ structure of the triplet charge separated excited state.}

$\begin{array}{lrrr}\mathrm{C} & 6.313449 & -2.478316 & -0.864007 \\ \mathrm{C} & 7.344476 & -3.274068 & -1.269658 \\ \mathrm{C} & 8.544006 & -2.482295 & -1.174917 \\ \mathrm{~N} & 8.235876 & -1.229578 & -0.727113 \\ \mathrm{C} & 6.885701 & -1.197109 & -0.544398 \\ \mathrm{C} & 6.780965 & 3.291268 & 1.002398 \\ \mathrm{C} & 5.898727 & 2.303354 & 0.676907 \\ \mathrm{C} & 6.685160 & 1.152556 & 0.318284 \\ \mathrm{~N} & 8.012357 & 1.447561 & 0.409099 \\ \mathrm{C} & 8.101763 & 2.744650 & 0.829084 \\ \mathrm{C} & 6.138054 & -0.085975 & -0.091581 \\ \mathrm{C} & 12.846173 & 2.849886 & 0.789119 \\ \mathrm{C} & 11.810965 & 3.653121 & 1.166952 \\ \mathrm{C} & 10.598445 & 2.943126 & 0.854691\end{array}$




\begin{tabular}{|c|c|c|c|}
\hline $\mathrm{N}$ & 10.903210 & 1.731198 & 0.307111 \\
\hline C & 12.264515 & 1.649637 & 0.247324 \\
\hline C & 9.297985 & 3.456068 & 1.072176 \\
\hline C & 12.344758 & -2.715330 & -1.616186 \\
\hline $\mathrm{C}$ & 13.229925 & -1.736294 & -1.273372 \\
\hline C & 12.456003 & -0.639251 & -0.754392 \\
\hline $\mathrm{N}$ & 11.129064 & -0.957576 & -0.78599 \\
\hline $\mathrm{C}$ & 11.031303 & -2.216060 & -1.30510 \\
\hline C & 9.837684 & -2.952228 & -1.49297 \\
\hline $\mathrm{C}$ & 13.011725 & 0.568093 & -0.27224 \\
\hline $\mathrm{Zn}$ & 9.566973 & 0.250300 & -0.20566 \\
\hline $\mathrm{H}$ & 5.263493 & -2.744504 & -0.7769 \\
\hline $\mathrm{H}$ & 7.276729 & -4.312936 & -1.58128 \\
\hline $\mathrm{H}$ & 6.532838 & 4.300849 & 1.318 \\
\hline $\mathrm{H}$ & 4.813953 & 2.362134 & 0.6632 \\
\hline $\mathrm{H}$ & 13.905592 & 3.072193 & 0.8834 \\
\hline $\mathrm{H}$ & 11.887241 & 4.637235 & 1.62104 \\
\hline $\mathrm{H}$ & 12.585196 & -3.685960 & -2.04101 \\
\hline $\mathrm{H}$ & 14.311068 & -1.778331 & -1.37225 \\
\hline C & 14.512843 & 0.679599 & -0.24240 \\
\hline $\mathrm{H}$ & 14.808910 & 1.727644 & -0.37405 \\
\hline $\mathrm{H}$ & 14.939543 & 0.145461 & -1.10074 \\
\hline C & 15.114140 & 0.132549 & 1.05448 \\
\hline $\mathrm{H}$ & 16.209258 & 0.232250 & 1.03745 \\
\hline $\mathrm{H}$ & 14.734731 & 0.677775 & 1.93122 \\
\hline $\mathrm{H}$ & 14.869655 & -0.931221 & 1.19019 \\
\hline C & 9.962803 & -4.367202 & -1.99066 \\
\hline $\mathrm{H}$ & 9.067530 & -4.637642 & -2.56408 \\
\hline $\mathrm{H}$ & 10.797580 & -4.436941 & -2.69974 \\
\hline $\mathrm{C}$ & 10.170261 & -5.370089 & -0.85307 \\
\hline $\mathrm{H}$ & 9.327707 & -5.351263 & -0.14632 \\
\hline $\mathrm{H}$ & 10.255146 & -6.389642 & -1.25628 \\
\hline $\mathrm{H}$ & 11.087113 & -5.147806 & -0.28757 \\
\hline $\mathrm{C}$ & 9.180378 & 4.831827 & $1.6707 \varepsilon$ \\
\hline $\mathrm{H}$ & 10.026449 & 5.451794 & 1.3497 \\
\hline $\mathrm{H}$ & 8.285645 & 5.330442 & 1.27726 \\
\hline $\mathrm{C}$ & 9.115620 & 4.795436 & 3.19974 \\
\hline $\mathrm{H}$ & 10.019525 & 4.336394 & 3.62668 \\
\hline $\mathrm{H}$ & 9.028094 & 5.815660 & 3.60066 \\
\hline $\mathrm{H}$ & 8.248147 & 4.215833 & 3.54792 \\
\hline C & 4.665496 & -0.219417 & -0.0532 \\
\hline C & 3.944645 & -0.472292 & -1.22964 \\
\hline C & 3.964502 & -0.070183 & 1.15245 \\
\hline C & 2.556105 & -0.543689 & -1.20304 \\
\hline $\mathrm{H}$ & 4.476939 & -0.583228 & -2.17715 \\
\hline C & 2.577891 & -0.166623 & 1.1774 \\
\hline $\mathrm{H}$ & 4.513753 & 0.110974 & 2.07918 \\
\hline C & 1.852854 & -0.388945 & -0.00108 \\
\hline $\mathrm{H}$ & 2.001828 & -0.718653 & -2.12776 \\
\hline $\mathrm{H}$ & 2.040603 & -0.055233 & 2.12180 \\
\hline $\mathrm{C}$ & 0.369791 & -0.437756 & 0.02552 \\
\hline C & -0.286833 & -1.528770 & 0.63625 \\
\hline C & -0.369346 & 0.620699 & -0.54929 \\
\hline $\mathrm{C}$ & -1.721458 & -1.537778 & 0.68910 \\
\hline
\end{tabular}




\begin{tabular}{|c|c|c|c|}
\hline C & 0.407481 & -2.644423 & 1.199063 \\
\hline C & -1.804870 & 0.565747 & -0.518505 \\
\hline C & 0.242151 & 1.765968 & -1.146476 \\
\hline C & -2.396393 & -2.623632 & 1.33329 \\
\hline $\mathrm{C}$ & -2.441238 & -0.498401 & 0.10830 \\
\hline C & -0.277605 & -3.672794 & 1.791392 \\
\hline $\mathrm{H}$ & 1.496769 & -2.675175 & 1.14872 \\
\hline C & -2.556108 & 1.619469 & -1.12462 \\
\hline C & -0.515959 & 2.764662 & -1.69942 \\
\hline $\mathrm{H}$ & 1.330018 & 1.844460 & -1.15608 \\
\hline C & -1.697168 & -3.663630 & 1.8711 \\
\hline $\mathrm{H}$ & -3.527584 & -0.518198 & $0.141 \mathrm{~s}$ \\
\hline $\mathrm{H}$ & 0.271443 & -4.517081 & 2.2141 \\
\hline $\mathrm{C}$ & -1.934439 & 2.690659 & -1.6999 \\
\hline $\mathrm{H}$ & -0.028983 & 3.632261 & -2.1495 \\
\hline $\mathrm{H}$ & -2.231927 & -4.477921 & 2.3 \\
\hline $\mathrm{H}$ & -2.530400 & 3.481678 & -2.158 \\
\hline C & -4.671082 & 1.874767 & 0.032 \\
\hline C & -4.569499 & 0.988588 & -2.3029 \\
\hline C & -6.145436 & 1.772657 & -0.01157 \\
\hline C & -6.047678 & 0.942527 & -2.33196 \\
\hline C & -6.786876 & 1.362562 & -1.20471 \\
\hline C & -6.897773 & 2.135063 & 1.09299 \\
\hline C & -6.703139 & 0.532013 & -3.48314 \\
\hline C & -8.202476 & 1.398162 & -1.27825 \\
\hline C & -8.300901 & 2.176109 & 1.01728 \\
\hline $\mathrm{H}$ & -6.381350 & 2.415743 & 2.01226 \\
\hline $\mathrm{C}$ & -8.103748 & 0.562378 & -3.55142 \\
\hline $\mathrm{H}$ & -6.107659 & 0.200675 & -4.33533 \\
\hline $\mathrm{C}$ & -8.945917 & 1.849266 & -0.16390 \\
\hline C & -8.848413 & 1.006577 & -2.47043 \\
\hline $\mathrm{H}$ & -8.898392 & 2.494409 & 1.87311 \\
\hline $\mathrm{H}$ & -8.628691 & 0.253959 & -4.45687 \\
\hline $\mathrm{C}$ & -10.412006 & 2.027875 & -0.2823 \\
\hline $\mathrm{C}$ & -10.322818 & 1.111265 & -2.5786 \\
\hline $\mathrm{N}$ & -11.001976 & 1.628767 & -1.4774 \\
\hline 0 & -11.075086 & 2.525500 & 0.60826 \\
\hline O & -10.919916 & 0.812376 & -3.59448 \\
\hline C & -12.446689 & 1.798044 & -1.61034 \\
\hline $\mathrm{H}$ & -12.813127 & 0.947678 & -2.1986 \\
\hline $\mathrm{H}$ & -12.866034 & 1.739483 & -0.5997 \\
\hline C & -12.817775 & 3.116946 & -2.2649 \\
\hline $\mathrm{H}$ & -12.397298 & 3.188116 & -3.2782 \\
\hline $\mathrm{H}$ & -13.911847 & 3.201040 & -2.3438 \\
\hline $\mathrm{H}$ & -12.453772 & 3.966641 & -1.668 \\
\hline $\mathrm{N}$ & -3.986621 & 1.516330 & -1.139 \\
\hline O & -4.059379 & 2.266341 & 1.003 \\
\hline O & -3.877776 & 0.628681 & -3.2314 \\
\hline C & -4.527066 & -2.797260 & $0.2255^{\prime}$ \\
\hline $\mathrm{C}$ & -4.336146 & -1.931308 & 2.5781 \\
\hline $\mathrm{C}$ & -5.915311 & -2.364126 & 0.19994 \\
\hline C & -5.742891 & -1.560466 & 2.53251 \\
\hline C & -6.490675 & -1.772729 & 1.35062 \\
\hline C & -6.678479 & -2.524137 & -0.97197 \\
\hline
\end{tabular}




$\begin{array}{lrrr}\mathrm{C} & -6.348089 & -0.967737 & 3.656798 \\ \mathrm{C} & -7.867015 & -1.388560 & 1.320060 \\ \mathrm{C} & -8.007961 & -2.143756 & -1.001914 \\ \mathrm{H} & -6.195563 & -2.962945 & -1.846136 \\ \mathrm{C} & -7.683094 & -0.606770 & 3.629274 \\ \mathrm{H} & -5.737286 & -0.808814 & 4.546549 \\ \mathrm{C} & -8.618540 & -1.596338 & 0.141156 \\ \mathrm{C} & -8.454737 & -0.816385 & 2.471266 \\ \mathrm{H} & -8.615067 & -2.270747 & -1.898841 \\ \mathrm{H} & -8.167571 & -0.160316 & 4.498783 \\ \mathrm{C} & -10.035620 & -1.262877 & 0.115410 \\ \mathrm{C} & -9.875508 & -0.485012 & 2.471248 \\ \mathrm{~N} & -3.818777 & -2.530081 & 1.412295 \\ \mathrm{~N} & -10.582243 & -0.746442 & 1.293999 \\ \mathrm{O} & -3.602779 & -1.737823 & 3.538944 \\ \mathrm{O} & -3.950571 & -3.330088 & -0.714477 \\ \mathrm{O} & -10.741076 & -1.422438 & -0.879474 \\ \mathrm{O} & -10.456837 & -0.013241 & 3.444512 \\ \mathrm{C} & -12.021748 & -0.516401 & 1.328095 \\ \mathrm{H} & -12.337285 & -0.348716 & 0.293078 \\ \mathrm{H} & -12.192414 & 0.397165 & 1.909789 \\ \mathrm{C} & -12.774232 & -1.693446 & 1.924563 \\ \mathrm{H} & -13.855398 & -1.488415 & 1.927479 \\ \mathrm{H} & -12.602029 & -2.607098 & 1.336376 \\ \mathrm{H} & -12.458778 & -1.875745 & 2.961913 \\ & & & \end{array}$

Optimized double NDI acceptor ( $\left.\mathrm{DA}_{2}\right)$ structure of the singlet local excited state.

$\begin{array}{lrrr}\mathrm{C} & 6.026225 & -2.480868 & -0.311599 \\ \mathrm{C} & 6.949428 & -3.478982 & -0.428380 \\ \mathrm{C} & 8.248588 & -2.854376 & -0.444570 \\ \mathrm{~N} & 8.100023 & -1.490436 & -0.348753 \\ \mathrm{C} & 6.757717 & -1.236202 & -0.277873 \\ \mathrm{C} & 7.232798 & 3.479783 & 0.061484 \\ \mathrm{C} & 6.225101 & 2.556536 & -0.041575 \\ \mathrm{C} & 6.850778 & 1.261323 & -0.051595 \\ \mathrm{~N} & 8.207087 & 1.402269 & 0.033613 \\ \mathrm{C} & 8.468018 & 2.747850 & 0.106217 \\ \mathrm{C} & 6.153841 & 0.031976 & -0.164005 \\ \mathrm{C} & 13.179697 & 2.217685 & 0.390076 \\ \mathrm{C} & 12.257235 & 3.220649 & 0.449444 \\ \mathrm{C} & 10.963557 & 2.618096 & 0.246476 \\ \mathrm{~N} & 11.114869 & 1.263070 & 0.073844 \\ \mathrm{C} & 12.456407 & 0.990677 & 0.146277 \\ \mathrm{C} & 9.751759 & 3.335909 & 0.224663 \\ \mathrm{C} & 11.987198 & -3.693514 & -0.477446 \\ \mathrm{C} & 12.993299 & -2.776748 & -0.322884 \\ \mathrm{C} & 12.366993 & -1.487610 & -0.183779 \\ \mathrm{~N} & 11.008143 & -1.632090 & -0.247389 \\ \mathrm{C} & 10.749357 & -2.967097 & -0.428816 \\ \mathrm{C} & 9.465200 & -3.558610 & -0.526991 \\ \mathrm{C} & 13.067344 & -0.269641 & 0.000165 \\ \mathrm{Zn} & 9.607764 & -0.112556 & -0.131249 \\ \mathrm{H} & 4.946426 & -2.583081 & -0.239274\end{array}$




\begin{tabular}{|c|c|c|c|}
\hline $\mathrm{H}$ & 6.746947 & -4.546222 & -0.472855 \\
\hline $\mathrm{H}$ & 7.120678 & 4.560469 & 0.096279 \\
\hline $\mathrm{H}$ & 5.157915 & 2.746691 & -0.121665 \\
\hline $\mathrm{H}$ & 14.255261 & 2.319168 & 0.510968 \\
\hline $\mathrm{H}$ & 12.457322 & 4.274345 & 0.626998 \\
\hline $\mathrm{H}$ & 12.102402 & -4.766830 & -0.605409 \\
\hline $\mathrm{H}$ & 14.060569 & -2.981934 & -0.304880 \\
\hline C & 14.568355 & -0.346544 & 0.116859 \\
\hline $\mathrm{H}$ & 15.020191 & 0.582012 & -0.257633 \\
\hline $\mathrm{H}$ & 14.948667 & -1.137987 & -0.544652 \\
\hline C & 15.046300 & -0.605627 & 1.546577 \\
\hline $\mathrm{H}$ & 16.145100 & -0.666526 & 1.588801 \\
\hline $\mathrm{H}$ & 14.722241 & 0.200127 & 2.222667 \\
\hline $\mathrm{H}$ & 14.636521 & -1.549881 & 1.936006 \\
\hline $\mathrm{C}$ & 9.403965 & -5.060394 & -0.643257 \\
\hline $\mathrm{H}$ & 8.502273 & -5.353517 & -1.197410 \\
\hline $\mathrm{H}$ & 10.246562 & -5.414939 & -1.253457 \\
\hline $\mathrm{C}$ & 9.423445 & -5.768244 & 0.712757 \\
\hline $\mathrm{H}$ & 8.560666 & -5.468971 & 1.326749 \\
\hline $\mathrm{H}$ & 9.388635 & -6.861100 & 0.583452 \\
\hline $\mathrm{H}$ & 10.334629 & -5.519558 & 1.277179 \\
\hline C & 9.809928 & 4.830418 & 0.411838 \\
\hline $\mathrm{H}$ & 10.751740 & 5.221465 & 0.004855 \\
\hline $\mathrm{H}$ & 9.018135 & 5.304960 & -0.184796 \\
\hline C & 9.668628 & 5.255794 & 1.874373 \\
\hline $\mathrm{H}$ & 10.479038 & 4.834378 & 2.488139 \\
\hline $\mathrm{H}$ & 9.703541 & 6.352338 & 1.967628 \\
\hline $\mathrm{H}$ & 8.714869 & 4.906992 & 2.297975 \\
\hline C & 4.673476 & 0.091036 & -0.169528 \\
\hline C & 3.938535 & -0.356085 & -1.278103 \\
\hline C & 3.968909 & 0.599471 & 0.932547 \\
\hline $\mathrm{C}$ & 2.547792 & -0.297071 & -1.284770 \\
\hline $\mathrm{H}$ & 4.470431 & -0.740902 & -2.151260 \\
\hline $\mathrm{C}$ & 2.578191 & 0.651729 & 0.929445 \\
\hline $\mathrm{H}$ & 4.523818 & 0.943962 & 1.808254 \\
\hline C & 1.850231 & 0.205777 & -0.180451 \\
\hline $\mathrm{H}$ & 1.990801 & -0.640426 & -2.159890 \\
\hline $\mathrm{H}$ & 2.044257 & 1.040310 & 1.800019 \\
\hline C & 0.364861 & 0.267476 & -0.188938 \\
\hline C & -0.384647 & -0.797230 & 0.354991 \\
\hline C & -0.285546 & 1.389449 & -0.743950 \\
\hline C & -1.819874 & -0.736158 & 0.321799 \\
\hline C & 0.228495 & -1.950778 & 0.933090 \\
\hline C & -1.720381 & 1.443844 & -0.735787 \\
\hline C & 0.426220 & 2.492752 & -1.308708 \\
\hline $\mathrm{C}$ & -2.569110 & -1.836823 & 0.838328 \\
\hline C & -2.451075 & 0.381406 & -0.211972 \\
\hline C & -0.529464 & -2.972912 & 1.440489 \\
\hline $\mathrm{H}$ & 1.317773 & -2.006108 & 0.965151 \\
\hline $\mathrm{C}$ & -2.368745 & 2.601921 & -1.268085 \\
\hline $\mathrm{C}$ & -0.238903 & 3.573367 & -1.825491 \\
\hline $\mathrm{H}$ & 1.516889 & 2.462024 & -1.321939 \\
\hline C & -1.948754 & -2.922386 & 1.387381 \\
\hline $\mathrm{H}$ & -3.538750 & 0.417717 & -0.21634 \\
\hline
\end{tabular}




\begin{tabular}{|c|c|c|c|}
\hline $\mathrm{H}$ & -0.044562 & -3.845928 & 1.881638 \\
\hline $\mathrm{C}$ & -1.659441 & 3.637056 & -1.801561 \\
\hline $\mathrm{H}$ & 0.323096 & 4.405514 & -2.253988 \\
\hline $\mathrm{H}$ & -2.545731 & -3.751364 & 1.771494 \\
\hline $\mathrm{H}$ & -2.183148 & 4.509278 & -2.195846 \\
\hline $\mathrm{C}$ & -4.325393 & 3.129804 & 0.029671 \\
\hline C & -4.509964 & 1.893360 & -2.131004 \\
\hline $\mathrm{C}$ & -5.759369 & 2.861059 & 0.288362 \\
\hline C & -5.936538 & 1.619363 & -1.833571 \\
\hline $\mathrm{C}$ & -6.515153 & 2.112134 & -0.641507 \\
\hline C & -6.346659 & 3.324750 & 1.452688 \\
\hline $\mathrm{C}$ & -6.692944 & 0.868401 & -2.716675 \\
\hline $\mathrm{C}$ & -7.883566 & 1.855873 & -0.376308 \\
\hline C & -7.705616 & 3.075811 & 1.710943 \\
\hline $\mathrm{H}$ & -5.736052 & 3.889916 & 2.158774 \\
\hline C & -8.050557 & 0.615578 & -2.452929 \\
\hline $\mathrm{H}$ & -6.217876 & 0.487446 & -3.622221 \\
\hline C & -8.468829 & 2.358377 & 0.806691 \\
\hline C & -8.644406 & 1.114196 & -1.306591 \\
\hline $\mathrm{H}$ & -8.180526 & 3.449521 & 2.619594 \\
\hline $\mathrm{H}$ & -8.658834 & 0.037849 & -3.150543 \\
\hline C & -9.912391 & 2.141782 & 1.064047 \\
\hline C & -10.090726 & 0.894155 & -1.067036 \\
\hline $\mathrm{N}$ & -10.628983 & 1.432630 & 0.099648 \\
\hline 0 & -10.462545 & 2.568673 & 2.058156 \\
\hline 0 & -10.783698 & 0.275308 & -1.850643 \\
\hline $\mathrm{C}$ & -12.074603 & 1.316986 & 0.300587 \\
\hline $\mathrm{H}$ & -12.395039 & 0.426074 & -0.249018 \\
\hline $\mathrm{H}$ & -12.238250 & 1.152467 & 1.372066 \\
\hline $\mathrm{C}$ & -12.819706 & 2.543858 & -0.193204 \\
\hline $\mathrm{H}$ & -12.657147 & 2.697532 & -1.270087 \\
\hline $\mathrm{H}$ & -13.899100 & 2.413357 & -0.028226 \\
\hline $\mathrm{H}$ & -12.501290 & 3.446658 & 0.347110 \\
\hline $\mathrm{N}$ & -3.799131 & 2.620969 & -1.167244 \\
\hline 0 & -3.624053 & 3.733918 & 0.809315 \\
\hline O & -3.962592 & 1.490449 & -3.132785 \\
\hline C & -4.552060 & -2.328273 & -0.448036 \\
\hline C & -4.713731 & -1.131217 & 1.734242 \\
\hline C & -6.030971 & -2.311956 & -0.550466 \\
\hline C & -6.192514 & -1.132200 & 1.606189 \\
\hline C & -6.803285 & -1.749360 & 0.490305 \\
\hline C & -6.652619 & -2.889706 & -1.644071 \\
\hline C & -6.972802 & -0.569265 & 2.601114 \\
\hline C & -8.217530 & -1.828181 & 0.425609 \\
\hline C & -8.055311 & -2.963082 & -1.708785 \\
\hline $\mathrm{H}$ & -6.033315 & -3.307911 & -2.439426 \\
\hline C & -8.375937 & -0.655622 & 2.541074 \\
\hline $\mathrm{H}$ & -6.479319 & -0.085176 & 3.445491 \\
\hline C & -8.830279 & -2.459643 & -0.679092 \\
\hline $\mathrm{C}$ & -8.992700 & -1.296763 & 1.480929 \\
\hline $\mathrm{H}$ & -8.552869 & -3.437318 & -2.556441 \\
\hline $\mathrm{H}$ & -8.996756 & -0.241623 & 3.337561 \\
\hline $\mathrm{C}$ & -10.304721 & -2.621056 & -0.713179 \\
\hline C & -10.466996 & -1.471610 & 1.473843 \\
\hline
\end{tabular}




$\begin{array}{lrrr}\mathrm{N} & -4.000959 & -1.795492 & 0.727555 \\ \mathrm{~N} & -11.022561 & -2.109430 & 0.368379 \\ \mathrm{O} & -4.137805 & -0.606525 & 2.661218 \\ \mathrm{O} & -3.842576 & -2.792235 & -1.311866 \\ \mathrm{O} & -10.869503 & -3.196392 & -1.619633 \\ \mathrm{O} & -11.162128 & -1.089672 & 2.394539 \\ \mathrm{C} & -12.467530 & -2.341750 & 0.371850 \\ \mathrm{H} & -12.807513 & -2.244937 & -0.666750 \\ \mathrm{H} & -12.915423 & -1.541647 & 0.971472 \\ \mathrm{C} & -12.831192 & -3.701213 & 0.941320 \\ \mathrm{H} & -13.923197 & -3.830991 & 0.925759 \\ \mathrm{H} & -12.384449 & -4.511933 & 0.348565 \\ \mathrm{H} & -12.493366 & -3.793769 & 1.983797\end{array}$

Optimized double NDI acceptor $\left(\mathrm{DA}_{2}\right)$ structure of the singlet charge separated excited state

\begin{tabular}{|c|c|c|c|}
\hline C & -6.239403 & 1.133037 & -2.327225 \\
\hline $\mathrm{C}$ & -7.249388 & 1.544105 & -3.145310 \\
\hline C & -8.479231 & 1.084295 & -2.552007 \\
\hline $\mathrm{N}$ & -8.209477 & 0.394415 & -1.40727 \\
\hline $\mathrm{C}$ & -6.854863 & 0.397609 & -1.2531 \\
\hline C & -6.885959 & -2.126056 & 2.768 \\
\hline C & -5.975920 & -1.593127 & 1.904 \\
\hline C & -6.728133 & -0.886765 & 0.901 \\
\hline $\mathrm{N}$ & -8.060966 & -1.002615 & 1.149 \\
\hline $\mathrm{C}$ & -8.189681 & -1.746419 & 2.287 \\
\hline C & -6.142384 & -0.205508 & -0.1931 \\
\hline C & -12.923964 & -1.455652 & 2.410 \\
\hline $\mathrm{C}$ & -11.913175 & -1.962484 & 3.1712 \\
\hline 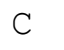 & -10.687545 & -1.697393 & 2.463 \\
\hline $\mathrm{N}$ & -10.960414 & -1.040762 & 1.3016 \\
\hline $\mathrm{C}$ & -12.315288 & -0.887335 & 1.235 \\
\hline $\mathrm{C}$ & -9.404061 & -2.077697 & 2.9258 \\
\hline $\mathrm{C}$ & -12.283052 & 1.350912 & -2.9517 \\
\hline $\mathrm{C}$ & -13.193316 & 0.907665 & -2.0393 \\
\hline $\mathrm{C}$ & -12.446544 & 0.303255 & -0.9668 \\
\hline $\mathrm{N}$ & -11.111949 & 0.391420 & -1.2304 \\
\hline C & -10.980689 & 1.023519 & -2.4328 \\
\hline $\mathrm{C}$ & -9.765215 & 1.347773 & -3.0775 \\
\hline $\mathrm{C}$ & -13.033222 & -0.282794 & 0.180 \\
\hline $\mathrm{Zn}$ & -9.584494 & -0.321074 & -0.0513 \\
\hline $\mathrm{H}$ & -5.175703 & 1.324478 & -2.4386 \\
\hline $\mathrm{H}$ & -7.148713 & 2.129244 & -4.055 \\
\hline $\mathrm{H}$ & -6.670248 & -2.726645 & 3.648 \\
\hline $\mathrm{H}$ & -4.894105 & -1.688816 & 1.937 \\
\hline $\mathrm{H}$ & -13.984496 & -1.475778 & 2.645 \\
\hline $\mathrm{H}$ & -12.012223 & -2.463907 & 4.1300 \\
\hline $\mathrm{H}$ & -12.498369 & 1.858352 & $-3.888 c$ \\
\hline $\mathrm{H}$ & -14.274087 & 0.993680 & -2.108 \\
\hline $\mathrm{C}$ & -14.530148 & -0.196888 & 0.314 \\
\hline $\mathrm{H}$ & -14.902814 & -1.066235 & 0.87 \\
\hline
\end{tabular}




\begin{tabular}{|c|c|c|c|}
\hline $\mathrm{H}$ & -14.994647 & -0.263353 & -0.677411 \\
\hline $\mathrm{C}$ & -14.977436 & 1.091302 & 1.008676 \\
\hline $\mathrm{H}$ & -16.073754 & 1.121272 & 1.087004 \\
\hline $\mathrm{H}$ & -14.558922 & 1.161492 & 2.023556 \\
\hline $\mathrm{H}$ & -14.649088 & 1.979222 & 0.448457 \\
\hline C & -9.844845 & 2.106154 & -4.375540 \\
\hline $\mathrm{H}$ & -8.979914 & 1.853828 & -5.002132 \\
\hline $\mathrm{H}$ & -10.723881 & 1.772213 & -4.942071 \\
\hline C & -9.909636 & 3.621011 & -4.168298 \\
\hline $\mathrm{H}$ & -9.020235 & 3.988878 & -3.635882 \\
\hline $\mathrm{H}$ & -9.964634 & 4.135291 & -5.138476 \\
\hline $\mathrm{H}$ & -10.793710 & 3.904815 & -3.578551 \\
\hline C & -9.325993 & -2.820476 & 4.232456 \\
\hline $\mathrm{H}$ & -10.214230 & -3.453874 & 4.351275 \\
\hline $\mathrm{H}$ & -8.473418 & -3.511296 & 4.212849 \\
\hline $\mathrm{C}$ & -9.196431 & -1.878423 & 5.431407 \\
\hline $\mathrm{H}$ & -10.057500 & -1.197054 & 5.497766 \\
\hline $\mathrm{H}$ & -9.143628 & -2.456231 & 6.365299 \\
\hline $\mathrm{H}$ & -8.287418 & -1.263705 & 5.356728 \\
\hline $\mathrm{C}$ & -4.665538 & -0.115575 & -0.219830 \\
\hline C & -3.927467 & -0.677204 & -1.271257 \\
\hline C & -3.980876 & 0.537398 & 0.815400 \\
\hline C & -2.539240 & -0.595218 & -1.279466 \\
\hline $\mathrm{H}$ & -4.446546 & -1.200027 & -2.077781 \\
\hline C & -2.594970 & 0.636370 & 0.790590 \\
\hline $\mathrm{H}$ & -4.543856 & 0.986979 & 1.636467 \\
\hline C & -1.853136 & 0.067000 & -0.252971 \\
\hline $\mathrm{H}$ & -1.971872 & -1.046957 & -2.096135 \\
\hline $\mathrm{H}$ & -2.071276 & 1.158847 & 1.594085 \\
\hline C & -0.372066 & 0.165937 & -0.262030 \\
\hline C & 0.241315 & 1.414826 & -0.505951 \\
\hline $\mathrm{C}$ & 0.407196 & -0.985297 & -0.009873 \\
\hline $\mathrm{C}$ & 1.673542 & 1.505733 & -0.474032 \\
\hline $\mathrm{C}$ & -0.494662 & 2.605452 & -0.795453 \\
\hline C & 1.839848 & -0.873026 & -0.016522 \\
\hline C & -0.161478 & -2.264222 & 0.276388 \\
\hline C & 2.307557 & 2.771674 & -0.691474 \\
\hline C & 2.432021 & 0.364276 & -0.235861 \\
\hline C & 0.149797 & 3.795388 & -1.007678 \\
\hline $\mathrm{H}$ & -1.583294 & 2.558773 & -0.850418 \\
\hline C & 2.634724 & -2.037486 & 0.221437 \\
\hline C & 0.635342 & -3.352985 & 0.512789 \\
\hline $\mathrm{H}$ & -1.247040 & -2.367801 & 0.311419 \\
\hline C & 1.566726 & 3.888387 & -0.947363 \\
\hline $\mathrm{H}$ & 3.516075 & 0.440407 & -0.218216 \\
\hline $\mathrm{H}$ & -0.430756 & 4.694402 & -1.226822 \\
\hline C & 2.050790 & -3.245305 & 0.478840 \\
\hline $\mathrm{H}$ & 0.182336 & -4.322448 & 0.731279 \\
\hline $\mathrm{H}$ & 2.068327 & 4.844762 & -1.099818 \\
\hline $\mathrm{H}$ & 2.676377 & -4.122059 & 0.654191 \\
\hline $\mathrm{C}$ & 4.712900 & -1.354670 & 1.264653 \\
\hline C & 4.674794 & -2.331361 & -1.042316 \\
\hline C & 6.184545 & -1.261142 & 1.179403 \\
\hline C & 6.151309 & -2.258790 & -1.07475 \\
\hline
\end{tabular}




\begin{tabular}{|c|c|c|c|}
\hline C & 6.858879 & -1.759415 & 0.039352 \\
\hline $\mathrm{C}$ & 6.905628 & -0.739380 & 2.242059 \\
\hline C & 6.838433 & -2.730855 & -2.185221 \\
\hline $\mathrm{C}$ & 8.276493 & -1.778573 & 0.025837 \\
\hline $\mathrm{C}$ & 8.309404 & -0.766994 & 2.232058 \\
\hline $\mathrm{H}$ & 6.358542 & -0.334833 & 3.095222 \\
\hline C & 8.238959 & -2.742864 & -2.198971 \\
\hline $\mathrm{H}$ & 6.261685 & -3.101880 & -3.034119 \\
\hline C & 8.989385 & -1.310949 & 1.152949 \\
\hline C & 8.954957 & -2.286828 & -1.101611 \\
\hline $\mathrm{H}$ & 8.887847 & -0.392151 & 3.078070 \\
\hline $\mathrm{H}$ & 8.793450 & -3.122438 & -3.058821 \\
\hline C & 10.460750 & -1.462758 & 1.208776 \\
\hline $\mathrm{C}$ & 10.432764 & -2.377223 & -1.094254 \\
\hline $\mathrm{N}$ & 11.080037 & -1.981208 & 0.075860 \\
\hline O & 11.103153 & -1.172950 & 2.200352 \\
\hline O & 11.060979 & -2.825941 & -2.031567 \\
\hline C & 12.531598 & -2.128320 & 0.103690 \\
\hline $\mathrm{H}$ & 12.893742 & -1.881712 & -0.902185 \\
\hline $\mathrm{H}$ & 12.909434 & -1.384487 & 0.814099 \\
\hline C & 12.966607 & -3.524540 & 0.509823 \\
\hline $\mathrm{H}$ & 12.590539 & -4.274470 & -0.201050 \\
\hline $\mathrm{H}$ & 14.064978 & -3.588149 & 0.522808 \\
\hline $\mathrm{H}$ & 12.599600 & -3.771859 & 1.517042 \\
\hline $\mathrm{N}$ & 4.061353 & -1.919388 & 0.153895 \\
\hline O & 4.075640 & -0.993945 & 2.229631 \\
\hline O & 4.011047 & -2.747507 & -1.965662 \\
\hline $\mathrm{C}$ & 4.451129 & 2.214594 & -1.632954 \\
\hline $\mathrm{C}$ & 4.244162 & 3.191742 & 0.680248 \\
\hline $\mathrm{C}$ & 5.850650 & 1.916674 & -1.357902 \\
\hline C & 5.663882 & 2.930763 & 0.888468 \\
\hline $\mathrm{C}$ & 6.422533 & 2.291630 & -0.118919 \\
\hline C & 6.624498 & 1.247263 & -2.322342 \\
\hline $\mathrm{C}$ & 6.266551 & 3.292930 & 2.106271 \\
\hline $\mathrm{C}$ & 7.805392 & 2.026357 & 0.115400 \\
\hline C & 7.963193 & 0.982766 & -2.089487 \\
\hline $\mathrm{H}$ & 6.140839 & 0.956072 & -3.255993 \\
\hline C & 7.609761 & 3.044665 & 2.328281 \\
\hline $\mathrm{H}$ & 5.643719 & 3.776763 & 2.860089 \\
\hline C & 8.568063 & 1.384072 & -0.885990 \\
\hline C & 8.388861 & 2.415114 & 1.341737 \\
\hline $\mathrm{H}$ & 8.583099 & 0.475524 & -2.829767 \\
\hline $\mathrm{H}$ & 8.097583 & 3.331067 & 3.261119 \\
\hline C & 9.995510 & 1.162872 & -0.686328 \\
\hline $\mathrm{C}$ & 9.818870 & 2.211072 & 1.564238 \\
\hline $\mathrm{N}$ & 3.728463 & 2.799553 & -0.573489 \\
\hline $\mathrm{N}$ & 10.536545 & 1.622559 & 0.518744 \\
\hline 0 & 3.506871 & 3.690801 & 1.513957 \\
\hline 0 & 3.880688 & 1.950411 & -2.679203 \\
\hline O & 10.706465 & 0.609672 & -1.520381 \\
\hline 0 & 10.393423 & 2.545649 & 2.592497 \\
\hline C & 11.978327 & 1.512041 & 0.701147 \\
\hline $\mathrm{H}$ & 12.315789 & 0.721302 & 0.022730 \\
\hline $\mathrm{H}$ & 12.152275 & 1.196504 & 1.737173 \\
\hline
\end{tabular}




$\begin{array}{lrrr}\mathrm{C} & 12.697921 & 2.813900 & 0.397430 \\ \mathrm{H} & 13.782868 & 2.686906 & 0.531752 \\ \mathrm{H} & 12.517391 & 3.128290 & -0.641622 \\ \mathrm{H} & 12.361079 & 3.612296 & 1.074071\end{array}$

\section{References}

1. Kelley, R. F.; Shin, W. S.; Rybtchinski, B.; Sinks, L. E.; Wasielewski, M. R. Photoinitiated charge transport in supramolecular assemblies of a 1,7,n,n`-tetrakis(zinc porphyrin)-perylene3,4:9,10-bis(dicarboximide). J. Am. Chem. Soc. 2007, 129, 3173-3181.

2. Phelan, B. T.; Schultz, J. D.; Zhang, J.; Huang, G.-J.; Young, Ryan M.; Wasielewski, M. R. Quantum coherence in ultrafast photo-driven charge separation. Faraday Discuss. 2019, 216, $319-338$.

3. Weller, A. Photoinduced electron transfer in solution: Exciplex and radical ion pair formation free enthalpies and their solvent dependence. Z. Physiol. Chem. 1982, 133, 93-98.

4. Kelley, R. F.; Tauber, M. J.; Wasielewski, M. R. Intramolecular electron transfer through the 20-position of a chlorophyll a derivative: An unexpectedly efficient conduit for charge transport. J. Am. Chem. Soc. 2006, 128, 4779-4791.

5. Thamyongkit, P.; Speckbacher, M.; Diers, J. R.; Kee, H. L.; Kirmaier, C.; Holten, D.; Bocian, D. F.; Lindsey, J. S. Swallowtail porphyrins: Synthesis, characterization and incorporation into porphyrin dyads. J. Org. Chem. 2004, 69, 3700-3710.

6. Marcus, R. A.; Sutin, N. Electron transfers in chemistry and biology. Biochim. Biophys. Acta 1985, $811,265-322$.

7. Fajer, J.; Borg, D. C.; Forman, A.; Dolphin, D.; Felton, R. H. Pi-cation radicals and dications of metalloporphyrins. J. Am. Chem. Soc. 1970, 92, 3451-3459. 
8. Hurley, J. K.; Sinai, N.; Linschitz, H. Actinometry in monochromatic flash photolysis: The extinction coefficient of triplet benzophenone and quantum yield of triplet zinc tetraphenyl porphyrin. Photochem. Photobiol. 1983, 38, 9-14.

9. Kikuchi, K.; Kurabayashi, Y.; Kokubun, H.; Kaizu, Y.; Kobayashi, H. Triplet yields of some tetraphenylporphyrins and octaethylporphyrins. J. Photochem. Photobiol., A 1988, 45, 261-63.

10. Klein, J. H.; Schmidt, D.; Steiner, U. E.; Lambert, C. Complete monitoring of coherent and incoherent spin flip domains in the recombination of charge-separated states of donor-iridium complex-acceptor triads. J. Am. Chem. Soc. 2015, 137, 11011-11021.

11. Fajer, J.; Borg, D.; Forman, A.; Adker, A.; Varadi, V. Cation radicals of tetraalkylprophyrins. J. Am. Chem. Soc. 1974, 96, 1238-1239.

12. Wu, Y.; Nalluri, S. K. M.; Young, R. M.; Krzyaniak, M. D.; Margulies, E. A.; Stoddart, J. F.; Wasielewski, M. R. Charge and spin transport in an organic molecular square. Angew. Chem., Int. Ed. 2015, 54, 11971-11977.

13. Batchelor, S.; Kay, C.; McLauchlan, K.; Shkrob, I. Time-resolved and modulation methods in the study of the effects of magnetic fields on the yields of free-radical reactions. J. Phys. Chem. 1993, 97, 13250-13258. 